\title{
SPECTRAL NORM OF A SYMMETRIC TENSOR AND ITS COMPUTATION
}

\author{
SHMUEL FRIEDLAND AND LI WANG
}

\begin{abstract}
We show that the spectral norm of a $d$-mode real or complex symmetric tensor in $n$ variables can be computed by finding the fixed points of the corresponding polynomial map. For a generic complex symmetric tensor the number of fixed points is finite, and we give upper and lower bounds for the number of fixed points. For $n=2$ we show that these fixed points are the roots of a corresponding univariate polynomial of degree at most $(d-1)^{2}+1$, except certain cases, which are completely analyzed. In particular, for $n=2$ the spectral norm of $d$-symmetric tensor is polynomially computable in $d$ with a given relative precision. For a fixed $n>2$ we show that the spectral norm of a $d$-mode symmetric tensor is polynomially computable in $d$ with a given relative precision with respect to the Hilbert-Schmidt norm of the tensor. These results show that the geometric measure of entanglement of $d$-mode symmetric qunits on $\mathbb{C}^{n}$ are polynomially computable for a fixed $n$.
\end{abstract}

2010 Mathematics Subject Classification. 13P15, 15A69, 65H04, 81P40.

Keywords: Symmetric tensors, homogeneous polynomials, spectral norm, anti-fixed and fixed points, computation of spectral norm, $d$-mode symmetric qubits, $d$-mode symmetric qunits on $\mathbb{C}^{n}$, geometric measure of entanglement.

\section{INTRODUCTION}

The spectral norm of a matrix, i.e., the maximal singular value, has numerous applications in pure and applied mathematics. One of the fundamental reasons for the tremendous use of this norm is that it is polynomial-time computable and the software for its computation is easily available on MAPLE, MATHEMATICA, MATLAB and other platforms.

Multiarrays, or $d$-mode tensors, i.e. $d \geqslant 3$, are starting to gain popularity due to data explosion and other applications. Usually, these problems deal with real valued tensors. Since the creation of quantum physics, $d$-mode tensors over complex numbers became the basic tool in treating the $d$-partite states. Furthermore, the special case of $d$-partite symmetric qubits, called bosons, is the basic ingredient in construction the boson sampling devices [1, 47.

The $(\mathbb{F}$-)spectral norm of a tensor is a well defined quantity over the real $(\mathbb{F}=\mathbb{R})$ or complex numbers $(\mathbb{F}=\mathbb{C})$. Unlike in the matrix case, the computation of the spectral norm in general can be NP-hard [26, 36]. Furthermore, the complex spectral norm of a real tensor can be bigger than its real spectral norm. In spite of these numerical difficulties, there is a need to compute these norms in special cases of interesting applications, as the geometric measure of entanglement. (See later in the Introduction and §2) Even the simplest case of $d$-partite qubits poses theoretical and numerical challenges [33. This can be partly explained by the fact that the space $\otimes d \mathbb{C}^{2}$ has dimension $2^{d}$.

In this paper we restrict ourselves to $d$-mode symmetric tensors over $\mathbb{F}^{n}$, denoted as $\mathrm{S}^{d} \mathbb{F}^{n}$. The dimension of this space is $\left(\begin{array}{c}n+d-1 \\ d\end{array}\right)=\left(\begin{array}{c}n+d-1 \\ n-1\end{array}\right)$. Hence for a fixed $n$ this dimension is $O\left(d^{n-1}\right)$. In particular, the dimension of $\mathrm{S}^{d} \mathbb{C}^{2}$ is $d+1$. A symmetric tensor $\mathcal{S} \in \mathrm{S}^{d} \mathbb{F}^{n}$ can be identified with a homogeneous polynomial $f=f_{\mathcal{S}}$ of degree $d$ in $n$ variables over $\mathbb{F}$, denoted here as $\mathrm{P}(d, n, \mathbb{F})$. It was already observed by J. J. Sylvester [53] that binary forms, i.e., $n=2$, posses very special properties related to polynomials of one complex variable. The spectral norm of $\mathcal{S} \in \mathrm{S}^{d} \mathbb{F}^{n}$ is denoted by $\|\mathcal{S}\|_{\sigma, \mathbb{F}}$. Its value is equal to the following maximum of $f \in \mathrm{P}(d, n, \mathbb{F})$ on the unit sphere in $\mathbb{F}^{n}$ :

$$
\|f\|_{\sigma, \mathbb{F}}=\max \left\{|f(\mathbf{x})|, \mathbf{x} \in \mathbb{F}^{n},\|\mathbf{x}\|=1\right\} .
$$

The spectral norm of a complex valued symmetric tensor is given as the maximum of the real part $\Re f$ over the set of complex valued vectors of norm one. The critical vector of $\Re f$ is an anti-eigenvector of $\mathbf{F}=\frac{1}{d} \nabla f$.

Date: January 11, 2020. 
The critical value is the eigenvalue of this system. In this paper we first convert this eigenvalue problem to the anti-fixed point $\mathbf{F}(\mathbf{x})=\overline{\mathbf{x}}$. Next we show that this anti-fixed point is a fixed point of another polynomial map $\mathbf{H}$, where $\mathbf{H}(\mathbf{x})=\overline{\mathbf{F}(\overline{\mathbf{F}(\mathbf{x})})}$. In the generic case, where the hypersurface $f(\mathbf{x})=0$ is a smooth hypersurface in the complex projective space, the number of fixed points is $(d-1)^{2 n}$, counted with multiplicities. In this case we find all fixed of $\mathbf{H}$ and compute the $\|f\|_{\sigma, \mathbb{C}}$. In the real case $\mathbb{F}=\mathbb{R}$, it is enough to consider the fixed points of $\mathbf{F}$. Our approach is completely different from the standard optimization methods that are used now in the literature [9, 15, 27, 29, 48, 57.

We now highlight the new and most important results of our paper. Associate with $\mathcal{S} \in \mathrm{S}^{d} \mathbb{C}^{n}$ the polynomial $f(\mathbf{x})=f_{\mathcal{S}}(\mathbf{x})=\mathcal{S} \times \otimes^{d} \mathbf{x}, \mathbf{x} \in \mathbb{C}^{n}$. (See the beginning of 92 for tensor notations.) Let $\mathbf{F}$ and $\mathbf{H}$ be defined as above. Each component of $\mathbf{F}$ and $\mathbf{H}$ is a homogeneous polynomial of degree $(d-1)$ and $(d-1)^{2}$ respectively. Theorem 7 shows that the complex spectral norm of $\mathcal{S} \in \mathrm{S}^{d} \mathbb{C}^{n}$ can be computed by finding the fixed points of the homogeneous polynomial map $\mathbf{H}: \mathbb{C}^{n} \rightarrow \mathbb{C}^{n}$, which is the polynomial system of equations $\mathbf{H}(\mathbf{x})-\mathbf{x}=\mathbf{0}$. Let $\mathrm{H}(d, n)$ be the classical hyperdeterminant variety in the space $\mathrm{P}(d, n, \mathbb{C})$ 31. Assume that $f \in \mathrm{P}(d, n, \mathbb{C}) \backslash \mathrm{H}(d, n)$. Then the number of fixed points of $\mathbf{F}$ and $\mathbf{H}$ is $(d-1)^{n}$ and $(d-1)^{2 n}$ respectively, (counted with multiplicities). If in addition $\mathcal{S}$ is real then its real spectral norm can be found by considering only the real fixed points of $\mathbf{F}$ in projective space $\mathbb{P} \mathbb{C}^{n}$.

Recall the elimination method for finding the roots of a system of $n$ polynomial equations in $n$ variables, each of degree at most $d$, with only isolated roots [58, 44]. Its complexity upper bound $d^{2^{n}}$ follows from Kronecker's work [34. If the system does not have roots at infinity then the solutions of this system have rational univariate representation. The arithmetic complexity of finding these roots is $O\left(d^{c n}\right)$ for some $c>0$ [50, 42. Using the above result, and the fact that the maps $\mathbf{F}$ or $\mathbf{H}$ corresponding to $f_{\mathcal{S}} \in \mathrm{P}(d, n, \mathbb{C}) \backslash \mathrm{H}(d, n)$ have a finite number of fixed points with a polynomial univariate representation, we give an algorithm to find the $\mathbb{F}$-spectral norm of $\mathcal{S} \in \mathrm{S}^{d} \mathbb{F}^{n}$ with an arbitrary relative precision with respect to the Hilbert-Schmidt norm of $\|\mathcal{S}\|$. See Theorems 12, 13 and 14. The arithmetic complexity of this algorithm is $O\left(d^{c n}\right)$. We remark that in all papers on complexity of finding the roots of zero dimensional zero set of polynomial equations in more than one variable cited in this paper we did not see any results on approximation of these roots within $\delta>0$ precision.

We study in detail the case of $d$-mode symmetric qubits, which are tensors in $\mathrm{S}^{d} \mathbb{C}^{2}$ of Hilbert-Schmidt norm one. We show that the nonzero fixed points of $\mathbf{H}$ can be computed by finding the roots of the corresponding polynomial of one complex variable of degree at most $(d-1)^{2}+1$, provided that this symmetric qubit is not in the exceptional family, (Theorem 18). We give a polynomial time algorithm for a relative approximation for all symmetric qubits, (Theorem [19). If $\mathcal{S}$ is real valued then its real spectral norm depends only on the real roots of this polynomial, or actually, on the real root of another polynomial of degree at most $d+1$.

Our results have an important application to the notion of the geometric measure of entanglement of $d$ partite symmetric states, (bosons), in quantum physics and its computation. Recall that a $d$-partite state is represented by a $d$-mode tensor $\mathcal{T}$ of Hilbert-Schmidt norm one: $\|\mathcal{T}\|=1$. One of the most important notion in quantum physics is the entanglement of $d$-partite systems [20, 51, 52, 7]. A state $\mathcal{T}$ is called entangled if it is not a product state, (rank one tensor). The distance of a state $\mathcal{T}$ to the product states is called the geometric measure of entanglement. It is given by $\sqrt{2\left(1-\|\mathcal{T}\|_{\sigma, \mathbb{C}}\right)}$, where $\|\mathcal{T}\|_{\sigma, \mathbb{C}}$ is the $\mathbb{C}$-spectral norm of $\mathcal{T}$. (See $\oint_{21}$. In particular, we deduce that the geometric measure of entanglement of a $d$-partite symmetric state $\mathcal{S} \in \mathrm{S}^{d} \mathbb{C}^{n}$, called a symmetric $d$-qunit, is polynomial time computable in $d$ for a fixed $n$. For symmetric qubits our results have much better complexity than in the case $n>2$.

We now survey briefly the contents of our paper. In $\$ 2$ we state our notations for tensors. We recall the definition of the spectral norm of a tensor $\mathcal{T}$. We state the well known connection between the notion of the geometric measure of entanglement and the spectral norm of the $d$-partite state (11).

In $₫ 3$ we first discuss the identification of $d$-symmetric tensors with the homogeneous polynomials of degree $d$. Then we study the spectral norm of $d$-symmetric tensors on $\mathbb{F}^{n}$. We recall the remarkable theorem of Banach [4] (7) that characterizes the spectral norm of a symmetric tensor, which was rediscovered a number of times in the mathematical and physical literature [9, 24, 38.

In $\$ 4$ we study the critical points of the homogeneous polynomial $f$ of degree $d$ on the unit sphere in $\mathbb{F}^{n}$. We call a symmetric tensor $\mathcal{S}$, where $f=f_{\mathcal{S}}$, singular if the system $\nabla f(\mathbf{x})=\mathbf{0}$ has a nontrivial solution in $\mathbb{C}^{n}$. Equivalently, if the corresponding hypersurface $f(\mathbf{x})=0$ in the projective space $\mathbb{P C}^{n}$ is singular. We show that the critical points of the real part of $f(\mathbf{x})$ correspond to anti-fixed points of $\mathbf{F}$ for $\mathbb{F}=\mathbb{C}$ : 
$\mathbf{F}(\mathbf{x})=\overline{\mathbf{x}}$ and to fixed points of $\pm \mathbf{F}$ for $\mathbb{F}=\mathbb{R}$. Theorem[7 explained above, outlines our theoretical approach for numerical computation of the spectral norm of symmetric tensors. Using the degree theory we give in Theorem 8 lower and upper bounds on the number of anti-fixed points of $\mathbf{F}$ corresponding to nonsingular $\mathcal{S} \in \mathrm{S}^{d} \mathbb{C}^{n}$.

In $\$ 5$ we study the available algorithms and their complexities to approximate the spectral norms of symmetric tensors in $\mathrm{S}^{d} \mathbb{F}^{n}$ for a fixed $n$. A symmetric tensor $\mathcal{S}$, and the corresponding $f_{\mathcal{S}} \in \mathrm{P}(d, n, \mathbb{C})$, are called strongly nonsingular if they are nonsingular and the coordinates $x_{1}$ of the $(d-1)^{2 n}$ fixed points of $\mathbf{H}$ are pairwise distinct. We show that most of the symmetric tensors in $S^{d} \mathbb{C}^{n}$ are strongly nonsingular. The fixed points of $\mathbf{H}$ corresponding to a strongly nonsingular tensor satisfy the conditions of the shape Lemma [50. In Theorem 12 we consider the case of a strongly nonsingular $\mathcal{T} \in \mathrm{S}^{d} \mathbb{Z}[\mathbf{i}]^{n}$, where $\mathbb{Z}[\mathbf{i}]$ are Gaussian integers. Assume that the coordinates of $\mathcal{T}$ are bounded in absolute value by $2^{\tau}$ for some $\tau \in \mathbb{N}$. Using recent results in [5] and [42] we show that the bit complexity of the computation of an approximation $L(\mathcal{T})$ to the norm $\|\mathcal{T}\|_{\sigma, \mathbb{C}}$ with a relative precision $2^{-e}, e \in \mathbb{N}$ is $\tilde{O}\left((\tau+e) d^{8 n}\right)$.

In Theorem 13 we discuss the computational complexity of an approximation $L(\mathcal{T})$ to a given $\mathcal{T} \in \mathrm{S}^{d} \mathbb{Z}[\mathbf{i}]^{n}$, without assuming that $\mathcal{T}$ is strongly nonsingular. We give a probabilistic algorithm to compute $L(\mathcal{T})$ with a similar bit complexity. Similar results are obtained in Theorem 14 for an approximation of the spectral norm of $\mathcal{T} \in \mathrm{S}^{d} \mathbb{Z}^{n}$ with slightly better complexity estimations. Theorems 1012 13, and 14 constitute the first major contribution of this paper.

An obvious question is what is the complexity of finding an approximation $L(\mathcal{T})$ to $\mathcal{T} \in \mathrm{S}^{d} \mathbb{Z}^{n}$ if we do not keep $n$ fixed. Theorem 15 shows that an approximation of the spectral norm of homogeneous quartic polynomials with an arbitrary precision is NP-hard. This result follows from the old result of Motzkin-Straus 45] relating the clique number of a graph to a certain maximum problem for the adjacency matrix of the graph, and its tensor interpretation to the spectral norm of tensors [26, (8.2)]. Unfortunately, it was not observed in 26 that the corresponding tensor is symmetric. Note that the approximation algorithm outlined in Theorem 14 has at least complexity $O\left(3^{n^{2}}\right)$, while the brute force method looking over all possible subsets of $n$ vertices of the given graph is $O\left(2^{n}\right)$.

In $\sqrt{6}$ we discuss in detail theoretical and numerical aspects of the computation of the spectral norm of $\mathcal{S} \in \mathrm{S}^{d} \mathbb{F}^{2}$. In Theorem 18 we show that the fixed points of the corresponding $\mathbf{H}$ in this case can be reduced to one polynomial equation of degree at most $(d-1)^{2}+1$, unless we are in the exceptional family. In the nonexceptional case we give a simple formula to compute the spectral norm. In Theorem 19 we give an approximation algorithm for the spectral norm of $\mathcal{T} \in \mathrm{S}^{d} \mathbb{Z}[\mathbf{i}]^{2}$ with a relative error $2^{-e}$ of bit complexity $\tilde{\mathcal{O}}\left(d^{2}\left(d^{4} \max \left(d^{2}, \tau\right)+e\right)\right)$. For $\mathcal{T} \in \mathrm{S}^{d} \mathbb{Z}^{2}$ we have better complexity results. Theorems 18 and 19 constitute the second main contribution of this paper.

In $\$ 7$ we analyze completely the exceptional family. We show how to obtain a relative approximation for symmetric tensors in this family. The complexity of this approximation is the same as for the nonexceptional family.

In $\$ 8$ we give numerical examples of our method for calculating the complex and the real spectral norm of some of $\mathcal{S} \in \mathrm{S}^{d} \mathbb{C}^{n}$ for $n=2,3,4$. Many of our examples correspond to polynomials that are sum of two monomials. Lemma 20 shows that in this case one can assume that the coefficients of these two monomials are nonnegative, if we compute the complex spectral norm. That is, the corresponding symmetric tensors have nonnegative entries. (This result is false if we consider the real spectral norm of real symmetric tensor which corresponds to a sum of two monomials.) Most of the examples of $d$-qubits considered in [3] are sums of two monomials. The authors believe that their examples for $d=4,5, \ldots, 12$ are the most entangled $d$-qubits. Our software confirms the values of the spectral norms of the examples in $[3]$. We also consider five one complex parameter families of these examples, and we compute a number of values of the spectral norms in these families. As expected, in all these computed examples the spectral norms are higher than in the examples in [3].

In Appendix 1 we consider a standard orthonormal basis in $\mathrm{S}^{d} \mathbb{C}^{n}$, the analog of Dicke states in $\mathrm{S}^{d} \mathbb{C}^{2}$ [18, and the entanglement of each element in the basis. We give an upper bound on the entanglement of symmetric states in $\mathrm{S}^{d} \mathbb{C}^{n}$.

In Appendix 2 we discuss the complexity results associated with a system of $m$ polynomial equations in $m$ variables with isolated roots and no roots at infinity. We recall simple necessary and sufficient conditions on such systems. We define an $x_{1}$-simple system which has only simple solutions with pairwise distinct $x_{1}$ 
coordinates. For $x_{1}$-simple systems the reduced Gröbner basis with respect to the order $x_{1} \prec \cdots \prec x_{m}$ satisfies the shape lemma. We recall the known complexity results of finding the reduced Gröbner basis in this case [5]. We also recall the complexity results for finding the roots of a polynomial in one complex variable [46]. Lemma 5 summarize the complexity results of finding all the roots of $x_{1}$-simple system with a given precision.

In Appendix 3 we discuss briefly the Majorana representation. This representation is used in physics literature [3, 43. We explain how Majorana representation suggests two kinds of the most entangled $d$ symmetric qubits, which solve either Tóths or Thomson's problems [56, 55]. Most of the example in [3] are based on these two problems. However, in certain cases as shown in [3] the most entagled symmetric states do not solve neither of the above problems.

\section{Spectral nORM AND ENTANGLEMEnt}

For a positive integer $d$, i.e., $d \in \mathbb{N}$, we denote by $[d]$ the set of consecutive integers $\{1, \ldots, d\}$. Let $\mathbb{F} \in\{\mathbb{R}, \mathbb{C}\}, \mathbf{n}=\left(n_{1}, \ldots, n_{d}\right) \in \mathbb{N}^{d}$. We will identify the tensor product space $\otimes_{i=1}^{d} \mathbb{F}^{n_{i}}$ with the space of $d$-arrays $\mathbb{F}^{\mathbf{n}}$. The entries of $\mathcal{T} \in \mathbb{F}^{\mathbf{n}}$ are denoted as $\mathcal{T}_{i_{1}, \ldots, i_{d}}$. We also will use the notation $\mathcal{T}=\left[\mathcal{T}_{i_{1}, \ldots, i_{d}}\right]$. So $\mathcal{T}$ is called a vector for $d=1$, a matrix for $d=2$ and a tensor for $d \geqslant 3$. Note that the dimension of $\mathbb{F}^{\mathbf{n}}$ is $N(\mathbf{n})=n_{1} \cdots n_{d}$.

Assume that $d \geqslant 2$ is an integer and $k \in\{0,1\}$. For $\mathbf{n}=\left(n_{1}, \ldots, n_{d}\right) \in \mathbb{N}^{d}$ let $\mathbf{m}=\left(n_{k+1}, \ldots, n_{d}\right) \in \mathbb{N}^{d-k}$. Assume that $\mathcal{T} \in \mathbb{F}^{\mathbf{n}}$ and $\mathcal{S} \in \mathbb{F}^{\mathbf{m}}$. Then $\mathcal{T} \times \mathcal{S}$ is the scalar $\sum_{i_{1}=\ldots, i_{d}}^{n_{1}, \ldots, n_{d}} \mathcal{T}_{i_{1}, \ldots, i_{d}} \mathcal{S}_{i_{1}, \ldots, i_{d}}$ for $k=0$ and a vector in $\mathbb{F}^{n_{1}}$, whose $i$-th coordinate is given by $(\mathcal{T} \times \mathcal{S})_{i}=\sum_{i_{2}=\ldots=i_{d}=1}^{n_{2}, \ldots, n_{d}} \mathcal{T}_{i, i_{2}, \ldots, i_{d}} \mathcal{S}_{i_{2}, \ldots, i_{d}}$, for $k=1$.

The inner product on $\mathbb{F}^{\mathbf{n}}$ is given as $\langle\mathcal{S}, \mathcal{T}\rangle:=\mathcal{S} \times \overline{\mathcal{T}}$, where $\overline{\mathcal{T}}=\left[\overline{\mathcal{T}_{i_{1}, \ldots, i_{d}}}\right]$. Furthermore, $\|\mathcal{S}\|=\sqrt{\langle\mathcal{S}, \mathcal{S}\rangle}$ is the Hilbert-Schmidt norm of $\mathcal{S}$. Assume that $\mathbf{x}_{i}=\left(x_{1, i}, \ldots, x_{n_{i}, i}\right)^{\top} \in \mathbb{F}^{n_{i}}$ for $i \in[d]$. Then $\otimes_{i=1}^{d} \mathbf{x}_{i}$ is a tensor in $\mathbb{F}^{\mathbf{n}}$, with the entries $\left(\otimes_{i=1}^{d} \mathbf{x}_{i}\right)_{i_{1}, \ldots, i_{d}}=x_{i_{1}, 1} \cdots x_{i_{d}, d} . \quad\left(\otimes_{i=1}^{d} \mathbf{x}_{i}\right.$ is called a rank one tensor if all $\mathbf{x}_{i} \neq \mathbf{0}$.) Assume that $\mathbf{x}_{1}=\cdots=\mathbf{x}_{d}=\mathbf{x}$. Then $\otimes{ }^{d} \mathbf{x}=\otimes_{i=1}^{d} \mathbf{x}_{i}$.

Denote the unit sphere in $\mathbb{F}^{n}$ by $\mathrm{S}(n, \mathbb{F})=\left\{\mathbf{x} \in \mathbb{F}^{n},\|\mathbf{x}\|=1\right\}$. Recall that the spectral norm of $\mathcal{T} \in \mathbb{F}^{\mathbf{n}}$ is given as

$$
\|\mathcal{T}\|_{\sigma, \mathbb{F}}=\max \left\{\left|\mathcal{T} \times \otimes_{i=1}^{d} \mathbf{x}_{i}\right|, \mathbf{x}_{i} \in \mathrm{S}\left(n_{i}, \mathbb{F}\right) \text { for } i \in[d]\right\} .
$$

Assume that $d=2$. Then $\mathcal{T}$ is a matrix $T \in \mathbb{F}^{n_{1} \times n_{2}}$. In that case $\|T\|_{\sigma, \mathbb{F}}$ is the spectral norm of $T$, and is equal to its maximum singular value $\sigma_{1}(T)$. In particular, for $T \in \mathbb{R}^{n_{1} \times n_{2}}$ one has equality $\|T\|_{\sigma, \mathbb{R}}=\|T\|_{\sigma, \mathbb{F}}$. Furthermore, as $\sigma_{1}(T)^{2}$ is the maximum eigenvalue of hermitian matrix $T T^{*}$ or $T^{*} T$. It is well known that $\sigma_{1}(T)$ can be computed in polynomial time in the entries of $T$ and $\max \left(n_{1}, n_{2}\right)$. See for example [32] for a general rectangular matrix, or 41 for direct of Lancos algorithm for $T T^{*}$. Another method for $T$, with Gaussian integer entries, is as follows: First compute the characteristic polynomial $p(z)$ of the hermitian matrix $H(T)=\left[\begin{array}{cc}0 & T \\ T^{*} & 0\end{array}\right]$. The complexity of such an algorithm is described in [19]. Next recall that the eigenvalues of $H(T)$ are $\pm \sigma_{i}(T)$ and 0 [25, Theorem 4.11.1]. Now use well known algorithms as [46] to approximate the roots $p(z)$.

In the rest of this paper we assume that $d \geqslant 3$, i.e., $\mathcal{T}$ is a tensor, unless stated otherwise. Unlike in the matrix case, for a real tensor $\mathcal{T} \in \mathbb{R}^{\mathbf{n}}$ it is possible that $\|\mathcal{T}\|_{\sigma, \mathbb{R}}<\|\mathcal{T}\|_{\sigma, \mathbb{C}}$ [26]. For simplicity of notation we will let $\|\mathcal{T}\|_{\sigma}$ denote $\|\mathcal{T}\|_{\sigma, \mathbb{C}}$, and no ambiguity will arise.

A standard way to compute $\|\mathcal{T}\|_{\sigma, \mathbb{F}}$ is an alternating maximization with respect to one variable, while other variables are fixed, see [15]. Other variants of this method is maximization on two variables using the SVD algorithms [27, or the Newton method [29, 57]. These methods in the best case yield a convergence to a local maximum, which provide a lower bound to $\|\mathcal{T}\|_{\sigma, \mathbb{F}}$. Semidefinite relaxation methods, as in [48, will yield an upper bound to $\|\mathcal{T}\|_{\sigma, \mathbb{F}}$, which will converge in some cases to $\|\mathcal{T}\|_{\sigma, \mathbb{F}}$.

Recall that in quantum physics $\mathcal{T} \in \mathbb{C}^{\mathbf{n}}$ is called a state if $\|\mathcal{T}\|=1$. (Furthermore, all tensors of the form $\zeta \mathcal{T}$, where $\|\mathcal{T}\|=1$ and $\zeta \in \mathbb{C},|\zeta|=1$ are identified as the same state. That is, the space of the states in $\mathbb{C}^{\mathbf{n}}$ is the quotient space $\mathrm{S}(N(\mathbf{n}), \mathbb{C}) / \mathrm{S}(1, \mathbb{C})$. For simplicity of our exposition will ignore this identification.) Denote by $\Pi^{\mathbf{n}}$ the product states in $\mathbb{C}^{\mathbf{n}}$ :

$$
\Pi^{\mathbf{n}}=\left\{\otimes_{i=1}^{d} \mathbf{x}_{i}, \mathbf{x}_{i} \in \mathrm{S}\left(n_{i}, \mathbb{C}\right), i \in[d]\right\} .
$$


The geometric measure of entanglement of a state $\mathcal{T} \in \mathbb{C}^{\mathbf{n}}$ is

$$
\operatorname{dist}\left(\mathcal{T}, \Pi^{\mathbf{n}}\right)=\min _{\mathcal{Y} \in \Pi^{\mathbf{n}}}\|\mathcal{T}-\mathcal{Y}\|
$$

As $\|\mathcal{T}\|=\|\mathcal{Y}\|=1$ it follows that $\operatorname{dist}\left(\mathcal{T}, \Pi^{\mathbf{n}}\right)=\sqrt{2\left(1-\|\mathcal{T}\|_{\sigma}\right)}$. Hence an equivalent measurement of entanglement is [33]

$$
\eta(\mathcal{T})=-\log _{2}\|\mathcal{T}\|_{\sigma}^{2}
$$

The maximal entanglement is

$$
\eta(\mathbf{n})=\max _{\mathcal{T} \in \mathbb{C}^{n},\|\mathcal{T}\|=1}-\log _{2}\|\mathcal{T}\|_{\sigma}^{2} .
$$

See [16] for other measurements of entanglement using the nuclear norm of $\mathcal{T}$. Lemma 9.1 in [26] implies

$$
\eta(\mathbf{n}) \leqslant \log _{2} N(\mathbf{n})
$$

Let $n^{\times d}=(n, \ldots, n) \in \mathbb{N}^{d}$. For $n=2$ we get that $\eta\left(2^{\times d}\right) \leqslant d$. In 39 it is shown that $\eta\left(2^{\times d}\right) \leqslant d-1$. A complementary result is given in [33]: For the set of states of Haar measure at least $1-e^{-d^{2}}$ on the sphere $\|\mathcal{T}\|=1$ in $\otimes^{d} \mathbb{C}^{2}$ the inequality $\eta(\mathcal{T}) \geqslant d-2 \log _{2} d-2$ holds. A generalization of this result to $\otimes^{d} \mathbb{C}^{n}$ is given in [17].

\section{Symmetric tensors}

A tensor $\mathcal{S}=\left[\mathcal{S}_{i_{1}, \ldots, i_{d}}\right] \in \otimes^{d} \mathbb{F}^{n}$ is called symmetric if $\mathcal{S}_{i_{1}, \ldots, i_{d}}=\mathcal{S}_{i_{\omega(1)}, \ldots, i_{\omega(d)}}$ for every permutation $\omega:[d] \rightarrow[d]$. Denote by $\mathrm{S}^{d} \mathbb{F}^{n} \subset \otimes^{d} \mathbb{F}^{n}$ the vector space of $d$-mode symmetric tensors on $\mathbb{F}^{n}$. In what follows we assume that $\mathcal{S}$ is a symmetric tensor and $d \geqslant 2$, unless stated otherwise. A tensor $\mathcal{S} \in \mathrm{S}^{d} \mathbb{F}^{n}$ defines a unique homogeneous polynomial of degree $d$ in $n$ variables $f(\mathbf{x})=\mathcal{S} \times \otimes^{d} \mathbf{x}$, where

$$
f(\mathbf{x})=\sum_{j_{k}+1 \in[d+1], k \in[n], j_{1}+\cdots+j_{n}=d} \frac{d !}{j_{1} ! \cdots j_{n} !} f_{j_{1}, \ldots, j_{n}} x_{1}^{j_{1}} \cdots x_{n}^{j_{n}} .
$$

Conversely, a homogeneous polynomial $f(\mathbf{x})$ of degree $d$ in $n$ variables defines a unique symmetric $\mathcal{S} \in \mathrm{S}^{d} \mathbb{F}^{n}$ as given in part (4) of Lemma 1 .

Hence it is advantageous to replace $\mathrm{S}^{d} \mathbb{F}^{n}$ by the isomorphic space of all homogeneous polynomials of degree $d$ in $n$ variables over $\mathbb{F}$, denoted as $\mathrm{P}(d, n, \mathbb{F})$. We now introduce the standard multinomial notation as in [49]. Let $\mathbb{Z}_{+}$be the set of all nonnegative integers. Denote by $J(d, n)$ be the set of all $\mathbf{j}=\left(j_{1}, \ldots, j_{n}\right) \in \mathbb{Z}_{+}^{n}$ appearing in the above definition of $f(\mathbf{x})$ :

$$
J(d, n)=\left\{\mathbf{j}=\left(j_{1}, \ldots, j_{n}\right) \in \mathbb{Z}_{+}^{n}, j_{1}+\cdots+j_{n}=d\right\} .
$$

It is well known that $|J(d, n)|=\left(\begin{array}{c}n+d-1 \\ n\end{array}\right)=\left(\begin{array}{c}n+d-1 \\ d-1\end{array}\right)$, see for example [4]. Define

$$
c(\mathbf{j})=\frac{d !}{j_{1} ! \cdots j_{n} !}
$$

For $\mathbf{x}=\left(x_{1}, \ldots, x_{n}\right)^{\top} \in \mathbb{F}^{n}$ and $\mathbf{j}=\left(j_{1}, \ldots, j_{n}\right) \in J(d, n)$ let $\mathbf{x}^{\mathbf{j}}$ be the monomial $x_{1}^{j_{1}} \cdots x_{n}^{j_{n}}$. Then the above definition of $f(\mathbf{x})$ is equivalent to

$$
f(\mathbf{x})=\sum_{\mathbf{j} \in J(d, n)} c(\mathbf{j}) f_{\mathbf{j}} \mathbf{x}^{\mathbf{j}}
$$

Let $\mathbf{U}$ and $\mathbf{V}$ be finite dimensional vector spaces over $\mathbb{F}$. Then $\mathbf{U}$ and $\mathbf{V}$ are isomorphic if and only if $\mathbf{U}$ and $\mathbf{V}$ have the same dimension. Assume that $\mathbf{U}$ and $\mathbf{V}$ are two inner product vector spaces over $\mathbb{F}$ of the same dimension $N$. Then $L: \mathbf{U} \rightarrow \mathbf{V}$ is called an isometry if $L$ preserves the inner product. Assume that $\mathbf{u}_{1}, \ldots, \mathbf{u}_{N}$ in $\mathbf{U}$ is an orthonormal basis in $\mathbf{U}$. Then a linear transformation $L: \mathbf{U} \rightarrow \mathbf{V}$ is an isometry if and only if $\mathbf{v}_{1}=L\left(\mathbf{u}_{1}\right), \ldots, \mathbf{v}_{N}=L\left(\mathbf{u}_{N}\right)$ is an orthonormal basis in $\mathbf{V}$.

The following lemma summarizes the properties of the isomorphisms of $\mathrm{S}^{d} \mathbb{F}^{n}$ to $\mathrm{P}(d, n, \mathbb{F})$ and to the auxiliary vector space $\mathbb{F}^{J(d, n)}$, and recalls Banach's characterization of the spectral norm of $\mathcal{S} \in \mathrm{S}^{d} \mathbb{F}^{n}$ as the maximum of the absolute value of the polynomial $\mathcal{S} \times\left(\otimes^{d} \mathbf{x}\right)$ on the unit sphere $\mathrm{S}(n, \mathbb{F})[4$ : 
Lemma 1. Let $\mathbb{F}^{J(d, n)}$ be the space of all vectors $\mathbf{f}=\left(f_{\mathbf{j}}\right), \mathbf{j} \in J(d, n)$. Assume that the inner product and the Hilbert norm on $\mathbb{F}^{J(d, n)}$ are given by

$$
\langle\mathbf{f}, \mathbf{g}\rangle=\sum_{\mathbf{j} \in J(d, n)} c(\mathbf{j}) f_{\mathbf{j}} \overline{g_{\mathbf{j}}}, \quad\|\mathbf{f}\|=\sqrt{\langle\mathbf{f}, \mathbf{f}\rangle}, \quad \mathbf{f}=\left(f_{\mathbf{j}}\right), \mathbf{g}=\left(g_{\mathbf{j}}\right) \in \mathbb{F}^{J(d, n)} .
$$

Then

(1) Let $\mathbf{e}_{\mathbf{j}}=\left(\delta_{\mathbf{j}, \mathbf{k}}\right)_{\mathbf{k} \in J(d, n)}, \mathbf{j} \in J(d, n)$, where $\delta_{\mathbf{j}, \mathbf{k}}$ is Kronecker's delta function, be the standard basis in $\mathbb{F}^{J(d, n)}$. Then $\frac{1}{\sqrt{c(\mathbf{j})}} \mathbf{e}_{\mathbf{j}}, \mathbf{j} \in J(d, n)$ is an orthonormal basis in $\mathbb{F}^{J(d, n)}$.

(2) $\mathbb{F}^{J(d, n)}$ is isomorphic to $\mathbb{F}^{\left(\begin{array}{c}n+d-1 \\ d-1\end{array}\right)}$. There is an isometry $L: \mathbb{F}^{J(d, n)} \rightarrow \mathbb{F}^{\left(\begin{array}{c}n+d-1 \\ d-1\end{array}\right)}$ which maps the orthonormal basis $\frac{1}{\sqrt{c(\mathbf{j})}} \mathbf{e}_{\mathbf{j}}, \mathbf{j} \in J(d, n)$ to the standard orthonormal basis in $\mathbb{F}^{\left(\begin{array}{c}n+d-1 \\ d-1\end{array}\right)}$.

(3) $\mathbb{F}^{J(d, n)}$ is isomorphic to $\mathrm{P}(n, d, \mathbb{F})$, where $\mathbf{f}$ corresponds to $f(\mathbf{x})$ given by (4)).

(4) The map $L: \mathrm{S}^{d} \mathbb{F}^{n} \rightarrow \mathrm{P}(d, n, \mathbb{F})$ which is given by $L(\mathcal{S})=f$, where $f(\mathbf{x})=S \times\left(\otimes^{d} \mathbf{x}\right)$, is an isomorphism and an isometry.

(5) Assume that $\mathcal{S} \in \mathrm{S}^{d} \mathbb{F}^{n}$ and let $\mathbf{F}(\mathbf{x})=\mathcal{S} \times\left(\otimes^{d-1} \mathbf{x}\right)$. Then

$$
\begin{aligned}
& \mathbf{F}(\mathbf{x})=\left(F_{1}(x), \ldots, F_{n}(\mathbf{x})\right)=\frac{1}{d} \nabla f(\mathbf{x})=\frac{1}{d}\left(\frac{\partial f}{\partial x_{1}}(\mathbf{x}), \ldots, \frac{\partial f}{\partial x_{n}}(\mathbf{x})\right), \\
& \sum_{i=1}^{n} x_{i} F_{i}(\mathbf{x})=f(\mathbf{x}) .
\end{aligned}
$$

(6) The spectral norm of a symmetric tensor is given by Banach's characterization [4]:

$$
\|\mathcal{S}\|_{\sigma, \mathbb{F}}=\max \left\{\frac{\left|\mathcal{S} \times\left(\otimes^{d} \mathbf{x}\right)\right|}{\|\mathbf{x}\|^{d}}, \mathbf{x} \in \mathbb{F}^{n} \backslash\{\mathbf{0}\}\right\}=\max \left\{\left|\mathcal{S} \times\left(\otimes^{d} \mathbf{x}\right)\right|, \mathbf{x} \in \mathrm{S}(n, \mathbb{F})\right\}, \quad \mathcal{S} \in \mathrm{S}^{d} \mathbb{F}^{n} .
$$

Proof. Parts (1)-(3) are straightforward.

Part (4). Let $\mathcal{S} \in \mathrm{S}^{d} \mathbb{F}^{n}$. Define $L(\mathcal{S})=f$, where $f(\mathbf{x})=\mathcal{S} \times\left(\otimes^{d} \mathbf{x}\right)$. Clearly $f \in \mathrm{P}(d, n, \mathbb{F})$. Assume that $f(\mathbf{x})$ is given by (44). For a given $i_{1}, \ldots, i_{d} \in[n]$ and $k \in[n]$ let $j_{k}$ be the number of times $k$ appears in the multiset $\left\{i_{1}, \ldots, i_{d}\right\}$. Set $\mathbf{j}=\left(j_{1}, \ldots, j_{n}\right)$. Then $L(\mathcal{S})_{\mathbf{j}}=f_{\mathbf{j}}$. It is straightforward to show that $L$ is an isomorphism. Furthermore, $\langle\mathcal{S}, \mathcal{T}\rangle=\langle L(\mathcal{S}), L(\mathcal{T})\rangle$. Hence $L$ is an isometry.

Part (5). Observe that $\frac{\partial}{\partial x_{m}}\left(\mathcal{S}_{i_{1}, \ldots, i_{d}} \mathbf{x}^{\mathbf{j}}\right)$ is not zero if and only if $i_{l}=m$ for some $l \in[d]$. So assume that $i_{l}=m$. Since $\mathcal{S}$ is symmetric we can choose $l \in[d]$. Hence $d F_{m}=\frac{\partial f}{\partial x_{m}}$, and (5) holds. Equality (6) is Euler's formula for $f \in \mathrm{P}(d, n, \mathbb{F})$ and $\mathbf{F}=\frac{1}{d} \nabla f$.

Part (6) is Banach's theorem [4], see [26] for details.

Banach's theorem (7) was rediscovered several times since 1938. In quantum physics literature it appeared in [38, for the case $\mathbb{F}=\mathbb{C}$. In mathematical literature, for the case $\mathbb{F}=\mathbb{R}$, it appeared in [9, 24]. (Observe that a natural generalization of Banach's theorem to partially symmetric tensors is given in [24].)

In view of Lemma 1 it makes sense to introduce the spectral norm on $\mathbb{F}^{J(d, n)}$ and $\mathrm{P}(d, n)$ :

$$
\|\mathbf{f}\|_{\sigma, \mathbb{F}}=\|f\|_{\sigma, \mathbb{F}}=\max \left\{\frac{|f(\mathbf{x})|}{\|\mathbf{x}\|^{d}}, \mathbf{x} \in \mathbb{F} \backslash\{\mathbf{0}\}\right\}=\max \{|f(\mathbf{x})|, \mathbf{x} \in \mathrm{S}(n, \mathbb{F})\},
$$

where $f(\mathbf{x})$ is given by (4).

We denote by $\mathcal{S}(\mathbf{j}) \in \mathrm{S}^{d} \mathbb{C}^{n}$ the symmetric state corresponding to $\frac{1}{\sqrt{c(\mathbf{j})}} \mathbf{e}_{\mathbf{j}}, \mathbf{j} \in J(d, n)$. Note that $\mathcal{S}(\mathbf{j})$ corresponds to the monomial $\sqrt{c(\mathbf{j})} \mathbf{x}^{\mathbf{j}}$, i.e., $\mathcal{S}(\mathbf{j}) \times \otimes^{d} \mathbf{x}=\sqrt{c(\mathbf{j})} \mathbf{x}^{\mathbf{j}}$. We also let $\|f\|=\|\mathbf{f}\|$.

\section{Critical points of $\Re\left(\mathcal{S} \times \otimes^{d} \mathbf{x}\right)$ ON $\mathrm{S}(n, \mathbb{F})$}

Recall that the projective space $\mathbb{P} \mathbb{C}^{N}$ is the space of lines through the origin in $\mathbb{C}^{N}$. Thus a point in $\mathbb{P} \mathbb{C}^{N}$ is the equivalence class $[\mathbf{y}]=\left\{t \mathbf{y}, t \in \mathbb{C} \backslash\{0\}, \mathbf{y} \in \mathbb{C}^{N} \backslash\{\mathbf{0}\}\right\}$. Note that for each $[\mathbf{y}] \in \mathbb{P} \mathbb{C}^{N}$, the hyperplane $\left\{\mathbf{x} \in \mathbb{C}^{N},\langle\mathbf{x}, \mathbf{y}\rangle=0\right\}$ is independent of the representative corresponding to $[\mathbf{y}]$.

Recall that $\mathcal{S} \in \mathrm{S}^{d} \mathbb{C}^{n}$ is called nonsingular [28] if

$$
\mathcal{S} \times \otimes^{d-1} \mathbf{x}=\mathbf{0} \Rightarrow \mathbf{x}=\mathbf{0} .
$$


Otherwise $\mathcal{S}$ is called singular. A nonzero homogeneous polynomial $f(\mathbf{x})$ defines a hypersurface $H(f):=$ $\left\{\mathbf{x} \in \mathbb{C}^{n} \backslash\{\mathbf{0}\}, f(\mathbf{x})=0\right\}$ in $\mathbb{P C}^{n} . H(f)$ is called a smooth hypersurface if $\nabla f(\mathbf{x}) \neq \mathbf{0}$ for each $\mathbf{x} \neq \mathbf{0}$ that satisfies $f(\mathbf{x})=0$.

Proposition 2. Assume that $\mathcal{S} \in \mathrm{S}^{d} \mathbb{C}^{n}$. Let $f(\mathbf{x})=\mathcal{S} \times \otimes^{d} \mathbf{x}$. Then $\mathcal{S}$ is nonsingular if and only if $H(f)$ is a smooth hypersurface in $\mathbb{P C}^{n}$.

Proof. Let $\mathbf{F}=\frac{1}{d} \nabla f$. Assume that $\mathbf{F}(\mathbf{x})=\mathbf{0}$ for some $\mathbf{x} \neq \mathbf{0}$. Euler's identity yields that $f(\mathbf{x})=0$. Use part (5) of Lemma 1 to deduce the proposition.

The following result is well known [31]:

Proposition 3. Denote by $\mathbb{P} \mathbb{C}^{J(d, n)}$ the complex projective space corresponding to the affine space $\mathbb{C}^{J(d, n)}$. With each $[\mathbf{f}] \in \mathbb{P} \mathbb{C}^{J(d, n)}$ associate the hypersurface $f(\mathbf{x})=0$ in $\mathbb{P} \mathbb{C}^{n}$, where $f(\mathbf{x})$ is given by (4). Then the set of singular hypersurfaces is the hyperdeterminant variety $\mathrm{H}(d, n) \subset \mathbb{P} \mathbb{C}^{J(d, n)}$, which is the zero set of the hyperdeterminant polynomial on $\mathbb{C}^{J(d, n)}$.

Corollary 4. The set of singular symmetric tensors in $\mathrm{S}^{d} \mathbb{C}^{n}$ is the zero set $V(d, n) \subset \mathrm{S}^{d} \mathbb{C}^{n}$ of the polynomial on $\mathrm{S}^{d} \mathbb{C}^{n}$ which is induced by the hyperdeterminant polynomial on $\mathbb{C}^{J(d, n)}$.

For a complex number $z=x+\mathbf{i} y \in \mathbb{C}, x, y \in \mathbb{R}$ we denote $x=\Re z$ and $y=\Im z$. Fix $\mathbf{x} \in \mathbb{C}^{n}$ and let $\zeta \in \mathbb{C}$. Then $\mathcal{S} \times \otimes^{d}(\zeta \mathbf{x})=\zeta^{d}\left(\mathcal{S} \times \otimes^{d} \mathbf{x}\right)$. Hence there exists $\zeta \in \mathbb{C},|\zeta|=1$ such that $\left|\mathcal{S} \times \otimes^{d} \mathbf{x}\right|=\Re\left(\mathcal{S} \times \otimes^{d}(\zeta \mathbf{x})\right)$. Therefore for $\mathbb{F}=\mathbb{C}$ we can replace the characterization (7) with:

$$
\|\mathcal{S}\|_{\sigma}=\max _{\mathbf{x} \in \mathrm{S}(n, \mathbb{C})} \Re\left(\mathcal{S} \times \otimes^{d} \mathbf{x}\right), \text { for } \mathcal{S} \in \mathrm{S}^{d} \mathbb{C}^{n} .
$$

Let $f \in \mathrm{P}(d, n, \mathbb{F})$. Consider $\Re f \mid \mathrm{S}(n, \mathbb{F})$, the restriction of $\Re f$ to the sphere $\mathrm{S}(n, \mathbb{F})$. Suppose first that $\mathbb{F}=\mathbb{R}$. Then $\Re f|\mathrm{~S}(n, \mathbb{R})=f| \mathrm{S}(n, \mathbb{R})$. A point $\mathbf{x} \in \mathrm{S}(n, \mathbb{R})$ is called a critical point of $f \mid \mathrm{S}(n, \mathbb{R})$ if the directional derivative of $f$ at $\mathbf{x}$ in direction of each $\mathbf{y}$, where $\langle\mathbf{y}, \mathbf{x}\rangle=0$, is 0 . Assume that $\mathbf{x}$ is a critical point of $f \mid \mathrm{S}(n, \mathbb{R})$. Then $f(\mathbf{x})$ is called a critical value of $f \mid \mathrm{S}(n . \mathbb{R})$.

Assume now that $\mathbb{F}=\mathbb{C}$. View $\mathbb{C}^{n}$ as $\mathbb{R}^{2 n}$ by writing $\mathbf{z} \in \mathbb{C}^{n}$ as $\mathbf{z}=\mathbf{x}+\mathbf{i y}$, where $\mathbf{x}, \mathbf{y} \in \mathbb{R}^{n}$. Clearly $\Re f(\mathbf{z}), \mathbf{z} \in \mathbb{C}^{n}$ can be viewed as a homogeneous polynomial $g(\mathbf{x}, \mathbf{y})$ of degree $d$ in $2 n$ variables $(\mathbf{x}, \mathbf{y})$. We then identify $\mathrm{S}(n, \mathbb{C})$ with $\mathrm{S}(2 n, \mathbb{R})$. Then $\Re f \mid \mathrm{S}(n, \mathbb{C})$ is $g \mid \mathrm{S}(2 n, \mathbb{R})$. Thus a critical point of $\Re f \mid \mathrm{S}(n, \mathbb{C})$ is the critical point of $g \mid \mathrm{S}(2 n, \mathbb{R})$, and the critical value of $\Re f \mid \mathrm{S}(n, \mathbb{C})$ is the critical value of $g \mid \mathrm{S}(2 n, \mathbb{R})$. Note that the points $\mathbf{x} \in \mathrm{S}(n, \mathbb{F})$ where $\Re f \mid \mathrm{S}(n, \mathbb{F})$ is maximum or minimum are critical points, and the maximum and minimum values are critical values.

Lemma 5. Assume that $\mathcal{S} \in \mathrm{S}^{d} \mathbb{F}^{n}, d \geqslant 2$. A point $\mathbf{x} \in \mathrm{S}(n, \mathbb{F})$ is a critical point of $\Re f(\mathbf{x})$ on $\mathrm{S}(n, \mathbb{F})$ if and only if

$$
\mathcal{S} \times \otimes^{d-1} \mathbf{x}=\lambda \overline{\mathbf{x}}, \quad \mathbf{x} \in \mathrm{S}(n, \mathbb{F}), \lambda \in \mathbb{R},
$$

where $\overline{\mathbf{x}}$ denote the complex conjugate of $\mathbf{x}$. The number of critical values $\lambda$ satisfying (9) is finite.

Proof. First assume that $\mathbb{F}=\mathbb{R}$. Let $\mathbf{x} \in \mathrm{S}(n, \mathbb{R})$. First assume that $\mathbf{x}$ is a critical point of $f(\mathbf{z})=\mathcal{S} \times \otimes^{d} \mathbf{z}$ for $\mathbf{z} \in \mathrm{S}(n, \mathbb{R})$. Let $\mathbf{y} \in \mathbb{R}^{n}$ be orthogonal to $\mathbf{x}: \mathbf{y}^{\top} \mathbf{x}=0$. Then $\|\mathbf{x}+t \mathbf{y}\|=\sqrt{1+t^{2}\|\mathbf{y}\|^{2}}=1+O\left(t^{2}\right)$ for $t \in \mathbb{R}$. Clearly

$$
\mathcal{S} \times \otimes^{d}(\mathbf{x}+t \mathbf{y})=\mathcal{S} \times \otimes^{d} \mathbf{x}+t d \mathbf{y}^{\top}\left(\mathcal{S} \times \otimes^{d-1} \mathbf{x}\right)+O\left(t^{2}\right) .
$$

As $\mathbf{x}$ is a critical point of $\mathcal{S} \times \otimes^{d} \mathbf{z}$ for $\mathbf{z} \in \mathrm{S}(n, \mathbb{R})$ it follows that $\mathbf{y}^{\top}\left(\mathcal{S} \times \otimes^{d-1} \mathbf{x}\right)=0$ for each $\mathbf{y}$ orthogonal to $\mathbf{x}$. Hence $\mathcal{S} \times \otimes^{d-1} \mathbf{x}$ is colinear with $\mathbf{x}$. As $\overline{\mathbf{x}}=\mathbf{x}$ for each $\mathbf{x} \in \mathbb{R}^{n}$ we deduce (9). Similar arguments show that if (9) holds for $\mathbf{x} \in \mathrm{S}(n, \mathbb{R})$ then $\mathbf{x}$ is a critical point.

As $f(\mathbf{x})$ is a polynomial on $\mathbb{R}^{n}$ it follows that the set of critical points of $f \mid \mathrm{S}(n, \mathbb{R})$ is a real algebraic set. This algebraic set is a finite union of connected algebraic sets [14, Proposition 1.6]. On each connected algebraic set of critical points $f$ is a constant function, whose value on this set is a critical value. This proves that the number of critical values for $\mathbb{F}=\mathbb{R}$ is finite.

Second assume that $\mathbb{F}=\mathbb{C}$. View $\mathbb{C}^{n}$ as $2 n$-dimensional real vector space $\mathbb{R}^{2 n}$ with the standard inner product $\Re\left(\mathbf{y}^{*} \mathbf{x}\right)$, where $\mathbf{y}^{*}=\overline{\mathbf{y}}^{\top}$. Hence $\|\mathbf{x}\|=\sqrt{\Re\left(\mathbf{x}^{*} \mathbf{x}\right)}$. Assume that $\mathbf{x} \in \mathrm{S}(n, \mathbb{C})$ is a critical point 
of $\Re\left(\mathcal{S} \times \otimes^{d} \mathbf{z}\right)$ on $\mathrm{S}(n, \mathbb{C})$. Let $\mathbf{y} \in \mathbb{C}^{n}$ be orthogonal to $\mathbf{x}: \Re\left(\mathbf{y}^{*} \mathbf{x}\right)=\Re\left(\overline{\mathbf{y}}^{\top} \mathbf{x}\right)=0$. Then $\|\mathbf{x}+t \mathbf{y}\|=$ $\sqrt{1+t^{2}\|\mathbf{y}\|^{2}}=1+O\left(t^{2}\right)$ for $t \in \mathbb{R}$. Hence

$$
\Re\left(\mathcal{S} \times \otimes^{d}(\mathbf{x}+t \mathbf{y})\right)=\Re\left(\mathcal{S} \times \otimes^{d} \mathbf{x}\right)+t d \Re\left(\mathbf{y}^{\top}\left(\mathcal{S} \times \otimes^{d-1} \mathbf{x}\right)\right)+O\left(t^{2}\right) .
$$

As $\mathbf{x}$ is a critical point we deduce that

$$
0=\Re\left(\mathbf{y}^{\top}\left(\mathcal{S} \times \otimes^{d-1} \mathbf{x}\right)\right)=\Re\left(\mathbf{y}^{*}\left(\overline{\mathcal{S} \times \otimes^{d-1} \mathbf{x}}\right)\right) .
$$

Hence $\overline{\mathcal{S} \times \otimes^{d-1} \mathbf{X}}$ is $\mathbb{R}$-colinear with $\mathbf{x}$. Thus (9) holds. Vice versa, suppose that (9) holds. As $\lambda \in \mathbb{R}$ and $0=\Re\left(\mathbf{y}^{*} \mathbf{x}\right)=\Re\left(\mathbf{y}^{\top} \overline{\mathbf{x}}\right)=0$ the equality (10) yields that $\mathbf{x}$ is a critical point.

Since $q(\mathbf{x}):=\Re\left(\mathcal{S} \times \otimes^{d} \mathbf{x}\right)$ is a polynomial on $\mathbb{C}^{n} \sim \mathbb{R}^{2 n}$ it follows from the above arguments for $\mathbb{F}=\mathbb{R}$ that $q \mid \mathrm{S}(n, \mathbb{C})$ has a finite number of critical values.

Clearly, a maximum point of $\left|\mathcal{S} \times \otimes^{d} \mathbf{x}\right|$ on $\mathrm{S}(n, \mathbb{F})$ is a critical point of $\Re\left(\mathcal{S} \times \otimes^{d} \mathbf{x}\right)$ on $\mathrm{S}(n, \mathbb{F})$. Hence:

Corollary 6. Let $d, n \geqslant 2$ be integers.

(1) Assume that $\mathcal{S} \in \mathrm{S}^{d} \mathbb{R}^{n}$. Then there exists $\mathrm{x} \in \mathrm{S}(n, \mathbb{R})$ satisfying (9) such that $|\lambda|=\|\mathcal{S}\|_{\sigma, \mathbb{R}}$. Furthermore, $\|\mathcal{S}\|_{\sigma, \mathbb{R}}$ is the maximum of all $|\lambda|$ satisfying (9).

(2) Assume that $\mathcal{S} \in \mathrm{S}^{d} \mathbb{C}^{n}$. Then there exists $\mathbf{x} \in \mathrm{S}(n, \mathbb{C})$ satisfying (9) such that $\lambda=\|\mathcal{S}\|_{\sigma}$. Furthermore, $\|\mathcal{S}\|_{\sigma}$ is the maximum of all $|\lambda|$ satisfying (9)).

We call $\mathrm{x} \in \mathrm{S}(n, \mathbb{F})$ and $\lambda \in \mathbb{F}$ an eigenvector and an eigenvalue of $\mathcal{S} \in \mathrm{S}^{d} \mathbb{F}^{n}$ if the following conditions hold [8]:

$$
\mathcal{S} \times \otimes^{d-1} \mathbf{x}=\lambda \mathbf{x}, \quad \mathbf{x} \in \mathrm{S}(n, \mathbb{F}), \lambda \in \mathbb{F}, \quad \mathcal{S} \in \mathrm{S}^{d} \mathbb{F}^{n} .
$$

Assume that $\mathbb{F}=\mathbb{R}$. Then the above equality is equivalent to (9). First assume that $d$ is odd and $\mathbf{x}$ is an eigenvector of $\mathcal{S}$. Then $-\mathrm{x}$ is an eigenvector of $\mathcal{S}$ corresponding to $-\lambda$. Hence without loss of generality we can consider only nonnegative eigenvalues of $\mathcal{S}$. Second assume that $d$ is even and $\mathbf{x}$ is an eigenvector of $\mathcal{S}$. Then $-\mathbf{x}$ is also eigenvector of $\mathcal{S}$ corresponding to $\lambda$.

A vector $\mathbf{x} \in \mathrm{S}(n, \mathbb{C})$ and a scalar $\lambda \in \mathbb{R}$ that satisfy (9) are called the anti-eigenvector and anti-eigenvalue of $\mathcal{S} \in \mathrm{S}^{d} \mathbb{C}^{n}$. Note that if $\mathbf{x}$ is an anti-eigenvector and $\lambda$ a corresponding anti-eigenvalue then $\zeta \mathbf{x}$ is also antieigenvector with a corresponding anti-eigenvalue $\varepsilon \lambda$, where $\varepsilon= \pm 1$ and $\zeta^{d}=\varepsilon$. Hence, we can always assume that each nonzero anti-eigenvalue is positive, and there are $d$ different choices of $\zeta$ such that $\zeta \mathbf{x} \in \operatorname{span}(\mathbf{x})$ is an anti-eigenvector corresponding to a given positive anti-eigenvalue $\lambda$.

We now state the first main result of this paper, which gives the theoretical foundation for the computational methods of our paper.

Theorem 7. Let $\mathcal{S} \in \mathrm{S}^{d} \mathbb{F}^{n} \backslash\{0\}$ and $d \geqslant 3$. Associate with $\mathcal{S}$ the polynomial $f(\mathbf{x})=\mathcal{S} \times \otimes^{d} \mathbf{x} \in \mathrm{P}(d, n, \mathbb{F}) \backslash$ $\{0\}$. Let $\mathbf{F}=\frac{1}{d} \nabla f$. Denote

$$
\operatorname{fix}(\mathbf{F})=\left\{\mathbf{x} \in \mathbb{C}^{n}, \mathbf{F}(\mathbf{x})=\mathbf{x}\right\}, \quad \operatorname{afix}(\mathbf{F})=\left\{\mathbf{x} \in \mathbb{C}^{n}, \mathbf{F}(\mathbf{x})=\overline{\mathbf{x}}\right\},
$$

the set of fixed and antifixed points of $\mathbf{F}$ in $\mathbb{C}^{n}$ respectively. Let $\omega_{d-2}=e^{\pi \mathbf{i} /(d-2)},\left(\omega_{d-2}^{d-2}=-1\right)$. Assume that $f \in \mathrm{P}(d, n, \mathbb{R})$. Denote

$$
\operatorname{fix}_{\mathbb{R}}(\mathbf{F})=\left\{\begin{array}{l}
\operatorname{fix}(\mathbf{F}) \cap \mathbb{R}^{n} \text { if } d \text { is odd, } \\
\left(\operatorname{fix}(\mathbf{F}) \cup \bar{\omega}_{d-2} \operatorname{fix}(\mathbf{F})\right) \cap \mathbb{R}^{n} \text { if } d \text { is even. }
\end{array}\right.
$$

(1) Assume that $\mathbb{F}=\mathbb{R}$. Then

$$
\|\mathcal{S}\|_{\sigma, \mathbb{R}}=\|f\|_{\sigma, \mathbb{R}}=\max \left\{\frac{|f(\mathbf{x})|}{\|\mathbf{x}\|^{d}}, \mathbf{x} \in \operatorname{fix}_{\mathbb{R}}(\mathbf{F}) \backslash\{\mathbf{0}\}\right\} .
$$

(2) Let $\mathbb{F}=\mathbb{C}$. Denote by $\overline{\mathbf{F}}: \mathbb{C}^{n} \rightarrow \mathbb{C}^{n}$ the polynomial mapping given by the equality $\overline{\mathbf{F}}(\mathbf{x})=\overline{\mathbf{F}(\overline{\mathbf{x}})}$. Let $\mathbf{H}=\overline{\mathbf{F}} \circ \mathbf{F}$. Then the set of fixed points of fix $(\mathbf{H})=\left\{\mathbf{x} \in \mathbb{C}^{n}, \mathbf{H}(\mathbf{x})=\mathbf{x}\right\}$ contains afix $(\mathbf{F})$. Furthermore, $\mathbf{x}$ is a fixed point of $\mathbf{H}$ if and only if $(\mathbf{x}, \mathbf{y}) \in \mathbb{C}^{n} \times \mathbb{C}^{n}$ is a solution to the system:

$$
\mathbf{F}(\mathbf{x})-\mathbf{y}=0, \quad \begin{gathered}
\overline{\mathbf{F}}(\mathbf{y})-\mathbf{x}=0 . \\
8
\end{gathered}
$$




\section{Moreover}

$$
\|\mathcal{S}\|_{\sigma}=\|f\|_{\sigma}=\max \left\{\frac{|f(\mathbf{x})|}{\|\mathbf{x}\|^{d}}, \mathbf{x} \in \operatorname{fix}(\mathbf{H}) \backslash\{\mathbf{0}\}\right\} .
$$

(3) Assume that $\mathcal{S} \in \mathrm{S}^{d} \mathbb{C}^{n}$ is nonsingular. Then $\operatorname{fix}(\mathbf{F})$ and $\operatorname{fix}(\mathbf{H})$ have cardinalities $(d-1)^{n}$ and $(d-1)^{2 n}$ respectively, counted with multiplicities. The origin $\mathbf{x}=\mathbf{0}$ is a fixed point of $\mathbf{F}$ and $\mathbf{H}$ of multiplicity one. Let $\mathbf{x} \in \operatorname{fix}(\mathbf{F}) \backslash\{\mathbf{0}\}$ and $\mathbf{y} \in \operatorname{fix}(\mathbf{H}) \backslash\{\mathbf{0}\}$. Then $\phi \mathbf{x} \in \operatorname{fix}(\mathbf{F}) \backslash\{\mathbf{0}\}$ and $\psi \mathbf{y} \in \operatorname{fix}(\mathbf{H}) \backslash\{\mathbf{0}\}$ if and only if $\phi^{d-2}=1$ and $\psi^{(d-1)^{2}-1}=1$.

Proof. (1-2) Let

$$
\left\{\begin{array}{l}
\alpha_{\mathbb{R}}=\sup \left\{\frac{|f(\mathbf{z})|}{\|\mathbf{z}\|^{d}}, \mathbf{z} \in \text { fix }_{\mathbb{R}}(\mathbf{F}) \backslash\{\mathbf{0}\}\right\}, \\
\alpha_{\mathbb{C}}=\sup \left\{\frac{\mid f(\mathbf{z} \mid)}{\|\mathbf{z}\|^{d}}, \mathbf{z} \in \operatorname{afix}(\mathbf{F}) \backslash\{\mathbf{0}\}\right\} .
\end{array}\right.
$$

The characterization (8) yields that $\alpha_{\mathbb{F}} \leqslant\|f\|_{\sigma, \mathbb{F}}$. Corollary 6 claims that $\|\mathcal{S}\|_{\sigma, \mathbb{F}}=\left|\lambda^{\star}\right|$, where $\left|\lambda^{\star}\right|$ is the maximum of all $|\lambda|$ satisfying (9). As $f \neq 0$ it follows that $\lambda^{\star} \in \mathbb{R} \backslash\{0\}$. From the discussion before this theorem it follows that we can assume that $\lambda^{*}>0$ unless $d$ is even and $\mathbb{F}=\mathbb{R}$.

Assume that $\mathbf{u} \in \mathrm{S}(n, \mathbb{F})$ satisfies $\mathbf{F}(\mathbf{u})=\lambda^{\star} \overline{\mathbf{u}}$. (I.e. $\mathbf{u}$ satisfies (9) with $\lambda=\lambda^{\star}$ ). Then there exists a positive $t$ such that $\left|\lambda^{\star}\right| t^{d-1}=t$. Let $\mathbf{x}=t \mathbf{u}$. Then $\mathbf{F}(\mathbf{x})=\frac{\lambda^{\star}}{\left|\lambda^{\star}\right|} \overline{\mathbf{x}}$. First assume that $\lambda^{\star}>0$. Then $\mathbf{x} \in \operatorname{afix}(\mathbf{F})$. Furthermore, if $\mathbb{F}=\mathbb{R}$ then $\mathbf{x} \in \operatorname{fix}(\mathbf{F}) \cap \mathbb{R}^{n} \subseteq \operatorname{fix}_{\mathbb{R}}(\mathbf{F})$. Clearly, $\|\mathcal{S}\|_{\sigma, \mathbb{F}}=\left|\lambda^{\star}\right|=\frac{|f(\mathbf{x})|}{\|\mathbf{x}\|^{d}}$. Hence $\|\mathcal{S}\|_{\sigma, \mathbb{F}}=\alpha_{\mathbb{F}}$ in this case.

Second assume that $\mathbb{F}=\mathbb{R}, d$ is even and $\lambda^{\star}<0$. Then $\mathbf{F}(\mathbf{x})=-\mathbf{x}$ and $\mathbf{x} \in \mathbb{R}^{n}$. Define $\mathbf{y}=\omega_{d-2} \mathbf{x}$. Then $\mathbf{F}(\mathbf{y})=\mathbf{y}$. Clearly $\bar{\omega}_{d-2} \mathbf{y}=\mathbf{x} \in \mathbb{R}^{n}$. Hence $\mathbf{x} \in \operatorname{fix}_{\mathbb{R}}(\mathbf{F})$. As $\|\mathcal{S}\|_{\sigma, \mathbb{R}}=\left|\lambda^{\star}\right|=\frac{|f(\mathbf{x})|}{\|\mathbf{x}\|^{d}}$ we deduce that $\alpha_{\mathbb{R}}=\|\mathcal{S}\|_{\sigma, \mathbb{R}}$. This show the characterization (12).

Assume that $\mathbf{F}(\mathbf{x})=\overline{\mathbf{x}}$. Then

$$
\mathbf{H}(\mathbf{x})=\overline{\mathbf{F}}(\mathbf{F}(\mathbf{x}))=\overline{\mathbf{F}}(\overline{\mathbf{x}})=\overline{\mathbf{F}(\mathbf{x})}=\mathbf{x} .
$$

Hence $\operatorname{afix}(\mathbf{F}) \subseteq \operatorname{fix}(\mathbf{H})$. Similarly, $\mathbf{x} \in \operatorname{fix}(\mathbf{H})$ if and only if (13) holds. (Set $\mathbf{y}=\mathbf{F}(\mathbf{x})$.) Let $\beta$ be the right hand side of (14). By definition $\|f\|_{\sigma} \geqslant \beta$. As afix $(\mathbf{F}) \subseteq \operatorname{fix}(\mathbf{H})$ it follows that $\alpha_{\mathbb{C}} \leqslant \beta$. Hence $\|f\|_{\sigma}=\beta$ and (14) holds.

(3) Recall that $\operatorname{fix}(\mathbf{F})$ is the set of solutions of the system $\mathbf{F}(\mathbf{x})-\mathbf{x}=\mathbf{0}$. As $d \geqslant 3$ the highest homogeneous part of this system is $\mathbf{F}(\mathbf{x})=\mathcal{S} \times \otimes^{d-1} \mathbf{x}$. As $\mathcal{S}$ is nonsingular we deduce that $\mathbf{F}(\mathbf{x})=\mathbf{0}$ has the only solution $\mathbf{x}=\mathbf{0}$. That is, the system $\mathbf{F}(\mathbf{x})-\mathbf{x}=\mathbf{0}$ does not have solutions at infinity. Therefore the Bezout theorem yields that the number of solutions counting with multiplicities is $(d-1)^{n}=\prod_{i=1}^{n} \operatorname{deg} \mathbf{F}_{i}$. (See [23] for a proof using degree theory.)

Observe next that $\mathbf{0} \in \operatorname{fix}(\mathbf{F})$. As the Jacobian $D(\mathbf{F}(\mathbf{x})-\mathbf{x})$ is $-I$ at $\mathbf{x}=\mathbf{0}$ it follows that $\mathbf{0}$ is a fixed point of multiplicity one. Assume that $\mathbf{x} \in \operatorname{fix}(\mathbf{F}) \backslash\{\mathbf{0}\}$. Then $\mathbf{F}(\phi \mathbf{x})=\phi^{d-1} \mathbf{x}=\phi^{d-2}(\phi \mathbf{x})$. Hence $\phi \mathbf{x}$ is a nonzero fixed point of $\mathbf{F}$ if and only if $\phi^{d-2}=1$.

To show similar results for $\mathbf{H}$ we first have to show that $\mathbf{H}(\mathbf{x})=\mathbf{0}$ has the only solution $\mathbf{x}=\mathbf{0}$. As $\mathcal{S}$ is nonsingular it follows that $\overline{\mathcal{S}}$ is nonsingular. Thus

$$
\mathbf{H}(\mathbf{x})=\overline{\mathbf{F}}(\mathbf{F}(\mathbf{x}))=\mathbf{0} \Rightarrow \mathbf{F}(\mathbf{x})=\mathbf{0} \Rightarrow \mathbf{x}=\mathbf{0} .
$$

As $\operatorname{deg} \mathbf{H}_{i}=(d-1)^{2}$ for $i \in[n]$ we deduce the similar results for $\mathbf{H}$.

Thus our approach to compute the spectral norm of $\mathcal{S}$ is to compute the fixed points of $\mathbf{F}$ and $\mathbf{H}$ using the available software as Bertini [6] for polynomial system of equations, and then use (12) or (14). Note that to compute the fixed points of $\mathbf{H}$ we can use also the system (13).

In [37] the authors consider the dynamics of a special anti-holomorphic map of $\mathbb{C}$ of the form $z \mapsto \bar{z}^{d}+c$. They also note that the dynamics of the "squared" map is given by the holomorphic map $z \mapsto\left(z^{d}+\bar{c}\right)^{d}+c$. Thus the dynamics of the maps $\mathbf{x} \mapsto \overline{\mathbf{F}(\mathbf{x})}$ and its square $-\mathbf{H}$ are generalizations of the dynamics studied in 37.

Suppose that $\mathbb{F}=\mathbb{C}$. Assume that $\mathbf{x} \in \mathrm{S}(n, \mathbb{C})$ and $\lambda \in \mathbb{C}$ are an eigenvector and the corresponding eigenvalue of $\mathcal{S} \in \mathrm{S}^{d} \mathbb{C}^{n}$, i.e., (11) holds. Let $\zeta \in \mathbb{C},|\zeta|=1$. Then $\zeta \mathbf{x}$ is an eigenvector of $\mathcal{S}$ with the corresponding eigenvalue $\zeta^{d-2} \lambda$. Assume that $\lambda \neq 0$. For $d>2$ we can choose $\zeta$ of modulus 1 such that 
$\zeta^{d-2} \lambda=|\lambda|>0$. Furthermore, the number of such choices of $\zeta$ is $d-2$. In this context it is natural to consider the eigenspace $\operatorname{span}(\mathbf{x})$, to which correspond a unique eigenvalue $\lambda \geqslant 0$.

It is shown in [8] that the number of different eigenspaces of generic $\mathcal{S} \in \mathrm{S}^{d} \mathbb{C}^{n}$ is

$$
c(2, n)=n, \quad c(d, n)=\frac{(d-1)^{n}-1}{d-2} \text { for } d \geqslant 3 .
$$

For $d \geqslant 3$ and a nonsingular $\mathcal{S}$ this result follows from part (3) of Theorem 7 where we count the number of nonzero fixed points of $\mathbf{F}$. That is, each $\mathcal{S} \in \mathrm{S}^{d} \mathbb{C}^{n} \backslash V(d, n)$ has the above number of eigenspaces $\operatorname{span}(\mathbf{x}), \mathbf{x} \in$ $\mathrm{S}(n, \mathbb{C})$. The obvious question is: what is the maximal number of eigenspaces $\operatorname{span}(\mathbf{x})$ corresponding to $\mathbf{x} \in \mathrm{S}(n, \mathbb{R})$ for $\mathcal{S} \in \mathrm{S}^{d} \mathbb{R}^{n} \backslash V(d, n)$. Since $\mathcal{S} \times \otimes^{d} \mathbf{x}$ has at least two critical points on $\mathrm{S}(n, \mathbb{R})$ for $\mathcal{S} \neq 0$, corresponding to the maximum and minimum values, it follows that $\mathcal{S} \neq 0$ has at least one real eigenspace. In [2] the authors study the average number of critical points of a random homogeneous function $f(\mathbf{x})$ of degree $d$, where its coefficients are independent Gaussian random variables.

Assume that $d=2$. Then $\mathrm{P}(2, n, \mathbb{F})$ is the space of quadratic forms in $n$ variables on $\mathbb{F}$, which correspond to the space of symmetric matrices $\mathrm{S}^{2} \mathbb{F}^{n}$. That is $f(\mathbf{x})=\mathbf{x}^{\top} S \mathbf{x}$, where $S \in \mathbb{F}^{n \times n}$ is symmetric. For $\mathbb{F}=\mathbb{R}$ the critical points of $f(\mathbf{x})$ correspond to the eigenvalues of $S$. For $\mathbb{F}=\mathbb{C}$ recall Schur's theorem: There exists a unitary matrix $U \in \mathbb{C}^{n \times n}$ such that $U^{\top} S U=\operatorname{diag}\left(a_{1}, \ldots, a_{n}\right), a_{1} \geqslant \cdots \geqslant a_{n} \geqslant 0$, where $\operatorname{diag}\left(a_{1}, \ldots, a_{n}\right) \in \mathrm{S}^{2} \mathbb{C}^{n}$ is a diagonal matrix with the diagonal entries $a_{1}, \ldots, a_{n}$. As $\bar{U}$ is unitary it follows that $a_{i}=\sigma_{i}(S), i \in[n]$ are the singular values of $S$. Let $U=\left[\mathbf{u}_{1}, \cdots, \mathbf{u}_{n}\right]$. Then $S U=\bar{U} \operatorname{diag}\left(a_{1}, \ldots, a_{n}\right)$ which is equivalent to $S \mathbf{u}_{i}=a_{i} \overline{\mathbf{u}}_{i}, i \in[n]$, which is a special case of (9).

We now give an estimate of the number of different positive anti-eigenvalues for a nonsingular $\mathcal{S} \in \mathrm{S}^{d} \mathbb{C}^{n}$.

Theorem 8. Assume that $\mathcal{S} \in \mathrm{S}^{d} \mathbb{C}^{n}$ is nonsingular. Then the number of positive anti-eigenvalues with corresponding anti-eigenspaces is finite. This number $\mu(\mathcal{S})$, counting with multiplicities, satisfies the inequalities

$$
\frac{(d-1)^{n}-1}{d} \leqslant \mu(\mathcal{S}) \leqslant \frac{(d-1)^{2 n}-1}{(d-1)^{2}-1}=\sum_{k=0}^{n-1}(d-1)^{2 k} .
$$

Proof. Assume that $\mathcal{S} \in \mathrm{S}^{d} \mathbb{C}^{n}$ is nonsingular. First suppose that $d=2$. Schur's theorem implies that the number of different positive anti-eigenvalues of a complex symmetric matrix, which are the singular values of $\mathcal{S}$, is at most $n$. Hence our theorem holds.

Second suppose that $d>2$. Assume that $\mathbf{x} \in \mathrm{S}(n, \mathbb{C})$ is an anti-eigenvector with corresponding antieigenvalue $\lambda>0$. As in the proof of Theorem 7 we can assume that $\mathbf{y} \in \operatorname{afix}(\mathbf{F}) \backslash\{\mathbf{0}\}$. Recall that $\operatorname{afix}(\mathbf{F}) \backslash\{\mathbf{0}\} \subset \operatorname{fix}(\mathbf{H})$. Theorem 7 yields that fix $(\mathbf{H}) \backslash\{\mathbf{0}\}$ has cardinality $(d-1)^{2 n}-1$. The subspace spanned by $\mathbf{y} \in \operatorname{fix}(\mathbf{H}) \backslash\{\mathbf{0}\}$ contains $(d-1)^{2}-1$ fixed points corresponding to $\psi \mathbf{y}$, where $\psi^{(d-1)^{2}-1}=1$. This shows the upper bound in (15).

We now show the lower bound using the degree theory as in $[23$. Let $\mu=\min \{\|\mathbf{F}\|,\|\mathbf{x}\|=1\}$. As $\mathbf{F}(\mathbf{x})=$ $\mathbf{0} \Rightarrow \mathbf{x}=\mathbf{0}$ it follows that $\mu>0$. Let $\mathbf{G}_{t}(\mathbf{x})=\mathbf{F}(\mathbf{x})-\bar{t}(\mathbf{x})$ for $t \in[0,1]$. Then $\left\|\mathbf{G}_{t}(\mathbf{x})\right\| \geqslant \mu\|\mathbf{x}\|^{d}-t\|\mathbf{x}\|$.In particular, $\lim _{\|\mathbf{x}\| \rightarrow \infty}\left\|\mathbf{G}_{t}(\mathbf{x})\right\|=\infty$. Hence $\mathbf{G}_{t}: \mathbb{C}^{n} \rightarrow \mathbb{C}^{n}$ is a proper map. Let $\mathbb{C}^{n} \cup\{\infty\}$ be one point compactification of $\mathbb{C}^{n}$. So $\mathbb{C}^{n} \cup\{\infty\}$ is homeomorphic to the $2 n$ dimensional sphere $\mathrm{S}^{2 n}$. Thus $\mathbf{G}_{t}$ extends to a continuous map $\hat{\mathbf{G}}_{t}: \mathrm{S}^{2 n} \rightarrow \mathrm{S}^{2 n}$. Let $\operatorname{deg} \hat{\mathbf{G}}_{t}$ be the topological degree of $\hat{\mathbf{G}}_{t}$. The above arguments so that this topological degree is constant for $t \in[0,1]$. Hence $\operatorname{deg} \hat{\mathbf{G}}_{1}=\operatorname{deg} \hat{\mathbf{G}}_{0}=\operatorname{deg} \hat{\mathbf{F}}$. The topological degree $\hat{\mathbf{F}}$ is just the covering degree of the proper complex polynomial map $\mathbf{F}$, which is $(d-1)^{n}$. Hence the number of the antifixed points of $\mathbf{F}$ is at least $\operatorname{deg} \hat{\mathbf{G}}=(d-1)^{n}$. As $\mathbf{0}$ is a simple fixed point of $\mathbf{H}$, $\mathbf{0}$ is a simple zero of $\mathbf{G}$. Therefore $|\operatorname{afix}(\mathbf{F}) \backslash\{\mathbf{0}\}| \geqslant(d-1)^{n}-1$. Recall that if $\mathbf{x} \in \operatorname{afix}(\mathbf{F}) \backslash\{\mathbf{0}\}$ then $\zeta \mathbf{x} \in \operatorname{afix}(\mathbf{F}) \backslash\{\mathbf{0}\}$ for $\zeta^{d}=1$. This establishes the lower bound in (15).

Consider the following example: $f(\mathbf{x})=\sum_{i=1}^{n} x_{i}^{d}$. Then $\mathbf{F}(\mathbf{x})=\left(x_{1}^{d-1}, \ldots, x_{n}^{d-1}\right)^{\top}$. It is straightforward to show that $|\operatorname{afix}(\mathbf{F})|=(d+1)^{n}$. Furthermore the number of positive eigenvalues of the corresponding $\mathcal{S}$ is $\frac{(d+1)^{n-1}-1}{d}$.

In what follows we will need the following observation:

Lemma 9. Assume that $\mathcal{S} \in \mathrm{S}^{d} \mathbb{F}^{n} \backslash\{0\}$. Let $\mathbf{F}(\mathbf{z})=\mathcal{S} \times\left(\otimes^{d-1} \mathbf{z}\right)$ and $\mathbf{H}=\overline{\mathbf{F}} \circ \mathbf{F}$. Then

$$
\|\mathbf{F}(\mathbf{z})\| \leqslant\|\mathcal{S}\|_{\sigma, \mathbb{F}}\|\mathbf{z}\|^{d-1}, \quad\|\mathbf{H}(\mathbf{z})\| \leqslant\|\mathcal{S}\|_{\sigma, \mathbb{F}}^{d}\|\mathbf{z}\|^{(d-1)^{2}}, \quad \mathbf{z} \in \mathbb{F}^{n} .
$$


For $\mathbf{z} \in \mathrm{S}(n, \mathbb{F})$ satisfying $\left|\mathcal{S} \times \otimes^{d} \mathbf{z}\right|=\|\mathcal{S}\|_{\sigma, \mathbb{F}}$ equality holds in the above inequalities. Suppose furthermore that $d>2$ and $\mathbf{x} \in \mathbb{F}^{n} \backslash\{\mathbf{0}\}$ is a fixed point of $\mathbf{H}$. Then

$$
\|\mathbf{x}\|^{-(d-2)} \leqslant\|\mathcal{S}\|_{\sigma, \mathbb{F}},
$$

and this inequality is sharp.

Proof. Since $\mathbf{F}$ and $\mathbf{H}$ are homogeneous maps of degree $d-1$ and $(d-1)^{2}$ respectively, it is enough to prove the first two inequalities of our lemma for $\mathbf{z} \in \mathrm{S}(n, \mathbb{F})$. Assume that $\mathbf{z} \in \mathrm{S}(n, \mathbb{F})$. Let $\mathbf{w}=\mathcal{S} \times \otimes^{d-1} \mathbf{z}$. First assume that $\mathbf{w}=\mathbf{0}$. Then $\mathbf{F}(\mathbf{z})=\mathbf{H}(\mathbf{z})=\mathbf{0}$ and the first two inequalities of our lemma trivially hold. Second assume that $\mathbf{w} \neq \mathbf{0}$. Let $\mathbf{u}=\frac{1}{\|\mathbf{w}\|} \overline{\mathbf{w}}$. Hence

$$
\|\mathbf{F}(\mathbf{z})\|=\left|\mathcal{S} \times\left(\mathbf{u} \otimes\left(\otimes^{d-1} \mathbf{z}\right)\right)\right| \leqslant\|\mathcal{S}\|_{\sigma, \mathbb{F}} .
$$

This establishes the first inequality of our lemma. Clearly, $\|\overline{\mathcal{S}}\|_{\sigma, \mathbb{F}}=\|\mathcal{S}\|_{\sigma, \mathbb{F}}$. Hence

$$
\|\mathbf{H}(\mathbf{z})\|=\|\overline{\mathbf{F}}(\mathbf{F}(\mathbf{z}))\| \leqslant\|\mathcal{S}\|_{\sigma, \mathbb{F}}(\|\mathbf{F}(\mathbf{z})\|)^{d-1} \leqslant\|\mathcal{S}\|_{\sigma, \mathbb{F}}\left(\|\mathcal{S}\|_{\sigma, \mathbb{F}}\right)^{d-1}=\|\mathcal{S}\|_{\sigma, \mathbb{F}}^{d} .
$$

This establishes the second inequality of our lemma.

Suppose that $\left|\mathcal{S} \times \otimes^{d} \mathbf{z}\right|=\|\mathcal{S}\|_{\sigma, \mathbb{F}}$ for $\mathbf{z} \in \mathrm{S}(n, \mathbb{F})$. First assume that $\mathbb{F}=\mathbb{C}$. Hence there exists $\zeta \in \mathbb{C},|\zeta|=1$ such that $\mathbf{x}=\zeta \mathbf{z}$ satisfies (9) with $\lambda=\|\mathcal{S}\|_{\sigma}$. Clearly $\|\mathbf{F}(\mathbf{z})\|=\|\mathbf{F}(\mathbf{x})\|=\lambda=\|\mathcal{S}\|_{\sigma}$. Moreover

$$
\mathbf{H}(\mathbf{x})=\overline{\mathbf{F}}(\lambda \overline{\mathbf{x}})=\lambda^{d-1} \overline{\mathbf{F}}(\overline{\mathbf{x}})=\lambda^{d} \mathbf{x}=\|\mathcal{S}\|_{\sigma}^{d} \mathbf{x} .
$$

Hence $\|\mathbf{H}(\mathbf{z})\|=\|\mathbf{H}(\mathbf{x})\|=\|\mathcal{S}\|_{\sigma}^{d}$.

Second assume that $\mathbb{F}=\mathbb{R}$. Then $\mathbf{z} \in \mathrm{S}(n, \mathbb{R})$ is a critical point of $\mathcal{S} \times \otimes^{d} \mathbf{x}$ on $\mathrm{S}(n, \mathbb{R})$. Corollary 6 yields that $\mathcal{S} \times \otimes^{d-1} \mathbf{z}= \pm\|\mathcal{S}\|_{\sigma, \mathbb{R}} \mathbf{z}$. Hence $\|\mathbf{F}(\mathbf{z})\|=\|\mathcal{S}\|_{\sigma, \mathbb{R}}$ and $\|\mathbf{H}(\mathbf{z})\|=\|\mathcal{S}\|_{\sigma, \mathbb{R}}^{d}$.

Assume finally that $\mathbf{H}(\mathbf{x})=\mathbf{x}, \mathbf{x} \neq \mathbb{F}^{n} \backslash\{\mathbf{0}\}$. The second inequality of our lemma yields $\|\mathbf{x}\|=\|\mathbf{H}(\mathbf{x})\| \leqslant$ $\|\mathcal{S}\|_{\sigma, \mathbb{F}}^{d}\|\mathbf{x}\|^{(d-1)^{2}}$. Hence $\|\mathcal{S}\|_{\sigma, \mathbb{F}}^{d} \geqslant\|\mathbf{x}\|^{-(d-1)^{2}+1}=\|\mathbf{x}\|^{-d(d-2)}$ which yields the third inequality of our lemma. If $\mathbf{x}$ corresponds to the critical vector $\mathbf{z} \in \mathrm{S}(n, \mathbb{F})$ with the eigenvalue $\lambda$ satisfying $|\lambda|=\|\mathcal{S}\|_{\sigma, \mathbb{F}}$ then $\|\mathbf{x}\|^{-(d-2)}=\|\mathcal{S}\|_{\sigma, \mathbb{F}}$.

\section{Polynomial-time computability of the spectral norm of $\mathcal{S} \in \mathrm{S}^{d} \mathbb{F}^{n}$ FOR a fixed $n$}

In this section we assume that $d \geqslant 3$ and $\mathcal{S} \neq 0$. (For $d=2$, (matrices), the spectral norm is the maximal singular value of the matrix, which is polynomially computable.)

Furthermore we are going to use the results of Appendix 2.

Definition 10. A symmetric tensor $\mathcal{S} \in \mathrm{S}^{d} \mathbb{C}^{n}$, and the corresponding polynomial $f(\mathbf{x})=f_{\mathcal{S}}(\mathbf{x})=\mathcal{S} \times \otimes{ }^{d} \mathbf{x}$, are called strongly nonsingular if the following conditions hold: First, $d \geqslant 3$ and $\mathcal{S}$ is nonsingular, i.e. the hypersurface $f=0$ is smooth in $\mathbb{P C}^{n}$. Second, the $x_{1}$ coordinates of $(d-1)^{2 n}$ solutions $(\mathbf{x}, \mathbf{y})$ of the system (13) are distinct.

Lemma 11. The set of $f \in \mathrm{P}(d, n, \mathbb{C})$ which are not strongly nonsingular, denoted as $\mathrm{P}_{\text {ss }}(d, n, \mathbb{C})$, has the following structure: Identify $\mathrm{P}(d, n, \mathbb{C})$ with $\mathbb{C}^{J(d, n)}$ and $\mathrm{P}_{s s}(d, n, \mathbb{C})$ with $\mathrm{V}_{s s}(d, n)$. Then $\mathrm{V}_{s s}(d, n)$ is a disjoint union of hyperdeterminant variety $\mathrm{H}(d, n)$ and the set $\mathrm{V}_{s s}^{\prime}(d, n) \subset \mathbb{C}^{J(d, n)}$ which is characterized as follows. There exists a bihomogeneous polynomial $p(\mathbf{u}, \mathbf{v}),(\mathbf{u}, \mathbf{v}) \in C^{J(d, n)} \times C^{J(d, n)}$ of total degree $2((d-$ $\left.1)^{2 n}-1\right)$ and of degree $(d-1)^{2 n}-1$ in $\mathbf{u}$ and $\mathbf{v}$ such that $\mathbf{u} \in \mathrm{V}_{s s}^{\prime}(d, n)$ if and only if $\mathbf{u} \notin \mathrm{H}(d, n)$ and $p(\mathbf{u}, \overline{\mathbf{u}})=0$.

Proof. Let $f, g \in \mathrm{P}(d, n, \mathbb{C})$. Define $\mathbf{F}=\frac{1}{d} \nabla f, \mathbf{G}=\frac{1}{d} \nabla g$. Consider a generalization of the system (13):

$$
\mathbf{F}(\mathbf{x})-\mathbf{y}=\mathbf{0}, \quad \mathbf{G}(\mathbf{y})-\mathbf{x}=\mathbf{0} .
$$

As $d \geqslant 3$, the homogeneous part of the above system, i.e., the system (40), $\mathbf{F}(\mathbf{x})=\mathbf{0}, \mathbf{G}(\mathbf{y})=\mathbf{0}$ has a unique solution $\mathbf{x}=\mathbf{y}=\mathbf{0}$ if and only if $f=0$ and $g=0$ are smooth hypersurfaces. That is, $f, g \notin \mathrm{H}(d, n)$. In this case the system (15) has $D=(d-1)^{2 n}$ isolated solutions, counting with multiplicities, and no solution at infinity. (See Appendix 2). Thus if we find the reduced Gröbner basis with respect to the order

$$
x_{1} \prec \cdots \prec x_{n} \prec y_{1} \prec \cdots \prec y_{n}
$$

then the last polynomial is a monic polynomial $p_{1}\left(x_{1}\right)$ of degree $(d-1)^{2 n}$. We now show an example where $p_{1}\left(x_{1}\right)$ has $(d-1)^{2 n}$ distinct roots. 
Let $f=g=h$, where $h=\sum_{i=1}^{n} x_{i}^{d}$. Then the system (15) is the system (13). It splits to $n$ systems in $\left(x_{k}, y_{k}\right)$ for $k \in[n]$ :

$$
x_{k}^{d-1}=y_{k}, \quad y_{k}^{d-1}=x_{k}, \quad k \in[n] .
$$

Note that this system has $D$ distinct solutions. However $x_{1}$ in these solutions has only $(d-1)^{2}$ distinct values: 0 , and $(d-1)^{2}-1$ roots of unity. We now show how perturb $h$ so that the $x_{1}$-coordinates of the solutions of (15) for $f=g=h$ are distinct. Let $\mathbf{a}=\left(a_{2}, \ldots, a_{n}\right)^{\top} \in \mathbb{C}^{n-1}$ and denote $h_{\mathbf{a}}(\mathbf{x})=$ $\left(x_{1}+\sum_{j=2} a_{j} x_{j}\right)^{d}+\sum_{i=2}^{n} x_{i}^{d}$. It is straightforward to check that $h_{\mathbf{a}}$ is nonsingular. Assume that $\mathbf{a}$ is close to $\mathbf{0}$. Then the $D$ solutions of (15) corresponding to $h_{\mathbf{a}}$ are close the $D$ distinct solutions of (15) corresponding to $h_{\mathbf{0}}$. In particular each solution of of (15) corresponding to $h_{\mathbf{a}}$ is analytic in a in the neighborhood of $\mathbf{a}=\mathbf{0}$. It is left to show that we can choose $\mathbf{a}$ close to $\mathbf{0}$ such that $x_{1}(\mathbf{a})$ are all distinct. Let $\left(\mathbf{x}_{l}(\mathbf{a}), \mathbf{y}_{l}(\mathbf{a})\right)=\left(x_{1, l}(\mathbf{a}), \ldots, x_{n . l}(\mathbf{a}), y_{1, l}(\mathbf{a}), \ldots, y_{n, l}(\mathbf{a})\right)^{\top}$ be the $l$-analytic solution of the system (15) corresponding to $h_{\mathbf{a}}$ for $l \in[D]$. Clearly if $x_{1, p}(\mathbf{0}) \neq x_{1, q}(\mathbf{0})$ then $x_{1, p}(\mathbf{a}) \neq x_{1, q}(\mathbf{a})$ for a small $\mathbf{a}$. Thus we need to study the case where $x_{1, p}(\mathbf{0})=x_{1, q}(\mathbf{0})=\zeta$. That is, $\zeta$ is a root of $z^{(d-1)^{2}}-z=0$.

The two equations of the system (15) corresponding $F_{1}(\mathbf{x})-y_{1}=0$ and $G_{1}(\mathbf{y})-x_{1}=0$ is

$$
\left(x_{1}+\sum_{j=2} a_{j} x_{j}\right)^{d-1}-y_{1}=0, \quad\left(y_{1}+\sum_{j=2} a_{j} y_{j}\right)^{d-1}-x_{1}=0 .
$$

To find the $\nabla x_{1, p}(\mathbf{0})$ we can assume that $x_{i, l}(\mathbf{a})=\mathbf{x}_{i, l}(\mathbf{0})$ and $y_{i, l}(\mathbf{a})=y_{i, l}(\mathbf{0})$ for $i \geqslant 2$. Observe that $y_{i, l}(\mathbf{0})=x_{i, l}^{d-1}(\mathbf{0})$ for $i \in[n]$ and $l \in[D]$. First assume that $\mathbf{x}_{1, p}(\mathbf{0})=\zeta \neq 0$. Hence $\zeta^{(d-1)^{2}-1}=1$. Thus

$$
\begin{aligned}
& x_{1, p}(\mathbf{a})=\left(y_{1, p}(\mathbf{a})+\sum_{j=2}^{n} a_{j} y_{p, j}(\mathbf{a})\right)^{d-1}=y_{1, p}^{d-1}(\mathbf{a})+(d-1) \zeta^{d-2} \sum_{j=2}^{n} a_{j} y_{j, p}(\mathbf{0})+\mathcal{O}\left(\|\mathbf{a}\|^{2}\right)= \\
& \left(x_{1, p}(\mathbf{a})+\sum_{i=2}^{n} a_{i} x_{j, p}(\mathbf{a})\right)^{(d-1)^{2}}+(d-1) \zeta^{d-2} \sum_{j=2}^{n} a_{j} x_{j, p}^{d-1}(\mathbf{0})+\mathcal{O}\left(\|\mathbf{a}\|^{2}\right)= \\
& x_{1, p}^{(d-1)^{2}}(\mathbf{a})+(d-1)^{2} \zeta^{(d-1)^{2}-1} \sum_{j=2}^{n} a_{j} x_{j, p}(\mathbf{0})+(d-1) \zeta^{d-2} \sum_{j=2}^{n} a_{j} x_{j, p}^{d-1}(\mathbf{0})+\mathcal{O}\left(\|\mathbf{a}\|^{2}\right) .
\end{aligned}
$$

As $\zeta^{(d-1)^{2}-1}=1$ we obtain the equation

$$
x_{1, p}(\mathbf{a})-x_{1, p}(\mathbf{a})^{(d-1)^{2}}=(d-1) \sum_{j=2}^{n}\left((d-1) x_{j, p}(\mathbf{0})+\zeta^{d-2} x_{j, p}^{d-1}(\mathbf{0})\right) a_{j}+\mathcal{O}\left(\|\mathbf{a}\|^{2}\right) .
$$

Hence

$$
\nabla x_{1, p}(\mathbf{0})=-\frac{d-1}{d(d-2)} \sum_{j=2}^{n}\left((d-1) x_{j, p}(\mathbf{0})+\zeta^{d-2} x_{j, p}^{d-1}(\mathbf{0})\right) a_{j} .
$$

Recall that $x_{j, p}(\mathbf{0})^{(d-1)^{2}}=x_{j, p}(\mathbf{0})$ for $j \geqslant 2$ and $p \in[D]$. Hence, for generic real $a_{2}, \ldots, a_{n}$ the values of $\nabla x_{1, p}(\mathbf{0})$ are disrinct for all solution $\mathbf{x}_{p}(\mathbf{a})$ such that $x_{1, p}(\mathbf{0})=\zeta \neq 0$.

Assume $\zeta=0$. The above arguments yield that $\nabla x_{1, p}(\mathbf{a})=\mathbf{0}$. Similarly, $\nabla y_{1, p}(\mathbf{a})=\mathbf{0}$. Hence the power series of $x_{1, p}(\mathbf{a})$ and $x_{1, p}(\mathbf{a})$ start from at least a quadratic polynomial. As $x_{1, p}(\mathbf{a})=\left(y_{1, p}(\mathbf{a})+\right.$ $\left.\sum_{j=1}^{n} a_{j} y_{j, p}(\mathbf{a})\right)^{d-1}$ we deduce that the power series of $x_{1, p}(\mathbf{a})$ start with a homogeneous polynomial of degree $d-1$ of the form $\left(\sum_{j=2}^{n} a_{j} y_{j, p}(\mathbf{0})\right)^{d-1}$. Recall that $y_{j, p}^{(d-1)^{2}}=y_{j, p}(\mathbf{0})$ for $j \geqslant 2$ and $p \in[D]$. Hence for generic real $a_{2}, \ldots, a_{n}$ all polynomials $\left(\sum_{j=2}^{n} a_{j} y_{j, p}(\mathbf{0})\right)^{d-1}$ are different. Hence for a small real generic values of $a_{2}, \ldots, a_{n}$ we have that $p_{1}\left(x_{1}\right)$ have $D$ distinct roots. (Note that $x_{1}=0$ is always a root of $p_{1}\left(x_{1}\right)$.)

Assume that $f, g \in \mathrm{P}(n, d, \mathbb{C})$ are represented by $\mathbf{u}, \mathbf{v} \in \mathbb{C}^{J(d, n)}$. Suppose that $\mathbf{u}, \mathbf{v} \notin \mathrm{H}(d, n)$. Then $p_{1}\left(x_{1}\right)$ is a polynomial of degree $D$. Its coefficients are rational functions in $\mathbf{u}, \mathbf{v}$. By multiplying $p_{1}\left(x_{1}\right)$ by a corresponding polynomial in $\mathbf{u}, \mathbf{v}$ we obtain a polynomial $P_{1}\left(x_{1}\right)$ of degree $D$ whose coefficients are polynomials in $\mathbf{u}, \mathbf{v}$ each one of degree $D . P_{1}\left(x_{1}\right)$ will not have $D$ distinct roots if and only if the discriminant of $P_{1}\left(x_{1}\right)$ is zero. This discriminant is a polynomial $p(\mathbf{u}, \mathbf{v})$ of degree $2(D-1)$. It is not hard to see that $p(\mathbf{u}, \mathbf{v})$ is a bihomogeneous polynomial in $(\mathbf{u}, \mathbf{v})$ of degree $D-1$ in $\mathbf{u}$ and $\mathbf{v}$ respectively. 
Observe next that the system (13) corresponds to a point $(\mathbf{u}, \overline{\mathbf{u}})$. For a real $\mathbf{u}$ the system (15) corresponds to $f=g$. We showed that for a generic choice of $\mathbf{u} p(\mathbf{u}, \mathbf{u}) \neq 0$. Thus $V_{s s}(d, n)=\mathrm{H}(d, n) \cup \mathrm{V}_{s s}^{\prime}(d, n)$, where $\mathrm{V}_{s s}^{\prime}(d, n)=\left\{\mathbf{u} \in \mathbb{C}^{J(d, n)}, \mathbf{u} \notin \mathrm{H}(d, n), p(\mathbf{u}, \overline{\mathbf{u}})=0\right\}$.

Let $\mathbf{i}=\sqrt{-1} \in \mathbb{C}$, i.e., $\mathbf{i}^{2}=-1$. Denote by $\mathbb{Z}[\mathbf{i}]=\mathbb{Z}+\mathbf{i} \mathbb{Z} \subset \mathbb{C}$ the integral domain of Gaussian integers, and by $\mathbb{Q}[\mathbf{i}]$ the field of Gaussian rationals. Let $\mathbb{Z}^{n} \subset \mathbb{R}^{n}$ and $\mathbb{Z}[\mathbf{i}]^{n} \subset \mathbb{C}^{n}$ be the $\mathbb{Z}$ and $\mathbb{Z}[\mathbf{i}]$ modules of vectors with integer and Gaussian integer coordinates respectively. We now give an upper bound on the complexity of finding the spectral norm $\|\mathcal{S}\|_{\sigma}$, assuming first that $\mathcal{S}$ is strongly nonsingular and the entries of $\mathcal{S}$ are Gaussian rationals, i.e., $\mathcal{S} \in \mathrm{S}^{d} \mathbb{Q}[\mathbf{i}]^{n}$. (Note that the assumption that $\mathcal{S}$ is strongly nonsingular yields that $\mathcal{S} \neq 0$.) Equivalently, we can assume that $\mathcal{S}=\frac{1}{N} \mathcal{T}$, where $\mathcal{T}$ is a symmetric tensor with Gaussian integers entries $\mathcal{T} \in \mathrm{S}^{d} \mathbb{Z}[\mathbf{i}]^{n}$ and $N \in \mathbb{N}$. Thus it is enough to estimate the spectral norm of $\mathcal{T}$. We identify $\mathcal{T}$ with $f(\mathbf{x})=\mathcal{T} \times\left(\otimes^{d} \mathbf{x}\right)$. We assume that each coefficient $f_{\mathbf{j}}$ in (44) is $a_{\mathbf{j}}+\mathbf{i} b_{\mathbf{j}}$, where $a_{\mathbf{j}}, b_{\mathbf{j}} \in \mathbb{Z}$ and $\left|a_{\mathbf{j}}\right|,\left|b_{\mathbf{j}}\right| \leqslant 2^{\tau}$ for each $\mathbf{j} \in J(d, n)$ for a given integer $M \in \mathbb{N}$.

Next we compute $\mathbf{F}=\left(F_{1}, \ldots, F_{n}\right)=\frac{1}{d} \nabla f$. As $\mathbf{F}=\mathcal{T} \times \otimes^{d-1} \mathbf{x}$, it follows that the coefficient of each monomial in the coordinates of $\mathbf{F}$ is a Gaussian integer. Hence the bit length of an integer coefficient is $\tau$. As $\mathcal{T}$ is strictly nonsingular we deduce that the system (13) has exactly $D=(d-1)^{2 n}$ simple solutions. Furthermore, after finding the reduced Gröbner basis with respect to the lexicographical order (16) we have the conditions of Lemma 4, the Shape Lemma, in Appendix 2. That is, the reduced Gröbner basis is of the form

$$
p_{1}\left(x_{1}\right), x_{2}-p_{2}\left(x_{1}\right), \ldots, x_{n}-p_{n}\left(x_{1}\right), y_{1}-p_{n+1}\left(x_{1}\right), \ldots, y_{n}-p_{2 n}\left(x_{1}\right) .
$$

The degree of $p_{1}\left(x_{1}\right)$ is $D$, and $p_{1}\left(x_{1}\right)$ has simple zeros. The degree of each $p_{i}\left(x_{1}\right)$ is less than $D$ for $i>1$. The solutions of the system (13) are parametrized by the roots $x_{1}$ of $p_{1}\left(x_{1}\right)$. They are of the form $(\mathbf{x}, \mathbf{y})$, where $\mathbf{x}=\left(x_{1}, p_{2}\left(x_{1}\right), \ldots, p_{n}\left(x_{1}\right)^{\top}\right.$ and $\mathbf{y}=\left(p_{n+1}\left(x_{1}\right), \ldots, p_{2 n}\left(x_{1}\right)\right)^{\top}$. Recall that the fixed points of $\mathbf{H}$ are the $\mathbf{x}$ part of the solutions $(\mathbf{x}, \mathbf{y})$ of the solutions of (13). Let

$$
X=\left\{\left(x_{1}, p_{2}\left(x_{1}\right), \ldots, p_{n}\left(x_{1}\right)\right)^{\top} \in \mathbb{C}^{n}, p_{1}\left(x_{1}\right)=0\right\} .
$$

Thus $X$ is a parametrization of all fixed points of $\mathbf{H}$ for strongly nonsingular $\mathcal{T} \in \mathrm{S}^{d} \mathbb{C}^{n}$. In particular, $|X|=D$. Lemma 5 of Appendix 2 gives the bit complexity of computing the coordinate of each solution $(\mathbf{x}, \mathbf{y})$ of (13) with precision $2^{-\ell}$, for a given $\ell \in \mathbb{N}$.

Recall that for a nonzero fixed point $\mathbf{x}$ of $\mathbf{H}$ corresponding to $\mathcal{S}$ the third inequality of Lemma 9 holds. As $\|\mathcal{T}\|_{\sigma} \leqslant\|\mathcal{T}\|$ we obtain that a nonzero fixed point of $\mathbf{H}$ corresponding to $\mathcal{T}$ satisfies the inequality $\|\mathbf{x}\| \geqslant\|\mathcal{T}\|^{-\frac{1}{d-2}}$. Hence Lemma 9 yields

$$
\|\mathcal{T}\|_{\sigma}=\max \left\{\|\mathbf{x}\|^{-(d-2)}, \mathbf{x} \in X,\|\mathbf{x}\| \geqslant\|\mathcal{T}\|^{-\frac{1}{d-2}}\right\}, \quad f(\mathbf{y})=\mathcal{T} \times\left(\otimes^{d} \mathbf{x}\right) .
$$

Theorem 12. Let $d \geqslant 3$ be an integer. Assume that $\mathcal{T} \in \mathrm{S}^{d} \mathbb{Z}[\mathbf{i}]^{n}$, and each coordinate of $\mathcal{T}$ is bounded above by $2^{\tau}$ for some $\tau \in \mathbb{N}$. If $\mathcal{T}$ is strongly nonsingular then for a given $e \in \mathbb{N}$ we can compute rational $L(\mathcal{T})$ satisfying

$$
\left|\|\mathcal{T}\|_{\sigma}-L(\mathcal{T})\right| \leqslant 2^{-e}\|\mathcal{T}\|_{\sigma} .
$$

The bit complexity of computing $L(\mathcal{T})$ is $\tilde{O}\left((\tau+e) d^{8 n}\right)$

Proof. Recall that $\mathbf{x} \in X \backslash\{\mathbf{0}\}$ satisfies the inequality $\|\mathbf{x}\| \geqslant\|\mathcal{T}\|^{-\frac{1}{d-2}}$. As $\mathcal{T} \in \mathrm{S}^{d} \mathbb{Z}[\mathbf{i}]^{n} \backslash\{0\}$ it follows that

$$
1 \leqslant\|\mathcal{T}\| \leqslant \sqrt{\left(\begin{array}{c}
n+d-1 \\
d
\end{array}\right)} 2^{\tau} \leqslant(d+1)^{(n-1) / 2} 2^{\tau} .
$$

We compute the coordinates of $\mathbf{x} \in X \backslash\{\mathbf{0}\}$ with precision $2^{-(e+k)}$, where $k \in \mathbb{N}$ is specified below. This will give an approximation $\hat{\mathbf{x}}(\mathbf{x})=\left(\hat{x}_{1}, \ldots, \hat{x}_{n}\right)^{\top}$ of $\mathbf{x}$. Observe that $\|\mathbf{x}-\hat{\mathbf{x}}(\mathbf{x})\| \leqslant \sqrt{n} 2^{-(e+k)}$. We assume that

$$
\sqrt{n} 2^{-k} \leqslant 2^{-d}(d+1)^{-(n-1) / 2(d-2)} 2^{-\tau /(d-2)} .
$$

To satisfy the above inequality we choose

$$
k=\left\lceil d+(1 / 2) \log _{2} n+\frac{n-1}{2(d-2)} \log _{2}(d+1)+\tau /(d-2)\right\rceil .
$$


Hence for any nonzero fixed point one has the inequality

$$
\|\mathbf{x}-\hat{\mathbf{x}}(\mathbf{x})\| \leqslant \sqrt{n} 2^{-(e+k)} \leqslant 2^{-(e+d)}\|\mathcal{T}\|^{-\frac{1}{d-2}} \leqslant 2^{-(e+d)}\|\mathbf{x}\| .
$$

In particular $\left(1-2^{-(e+d)}\right)\|\mathbf{x}\| \leqslant\|\hat{\mathbf{x}}(\mathbf{x})\| \leqslant\left(1-2^{-(e+d)}\right)\|\mathbf{x}\|$. Let

$$
L(\mathcal{T})=\max \left\{\|\hat{\mathbf{x}}(\mathbf{x})\|^{-(d-2)}, \mathbf{x} \in X \backslash\{\mathbf{0}\}\right\} .
$$

We claim that the inequality (19) holds. For a nonzero fixed point of $\mathbf{H}$ we estimate $\left|\|\mathbf{x}\|^{-(d-2)}-\|\hat{\mathbf{x}}(\mathbf{x})\|^{-(d-2)}\right|$. First observe that

$$
\begin{aligned}
& \left|\|\mathbf{x}\|^{d-2}-\|\hat{\mathbf{x}}(\mathbf{x})\|^{d-2}\right| \leqslant|\|\mathbf{x}\|-\|\hat{\mathbf{x}}(\mathbf{x})\||(d-2) \max \left(\|\mathbf{x}\|^{d-3},\|\hat{\mathbf{x}}(\mathbf{x})\|^{d-3}\right) \leqslant \\
& 2^{-(e+d)}(d-2)\left(1+2^{-(e+d)}\right)^{d-2}\|\mathbf{x}\|^{d-2} .
\end{aligned}
$$

Hence

$$
\begin{aligned}
& \left|\|\mathbf{x}\|^{-(d-2)}-\|\hat{\mathbf{x}}(\mathbf{x})\|^{-(d-2)}\right|=\left|\|\mathbf{x}\|^{d-2}-\|\hat{\mathbf{x}}(\mathbf{x})\|^{d-2}\right|\|\mathbf{x}\|^{-(d-2)}\|\hat{\mathbf{x}}(\mathbf{x})\|^{-(d-2)} \leqslant \\
& 2^{-(e+d)}(d-2)\left(1+2^{-(e+d)}\right)^{d-2}\left(1-2^{-(e+d)}\right)^{-(d-2)}\|\mathbf{x}\|^{-(d-2)} .
\end{aligned}
$$

As $e \geqslant 1$ and $d \geqslant 3$ it follows that $\left(1+2^{-(e+d)}\right) /\left(1-2^{-(e+d)}\right) \leqslant 17 / 15$. It is straightforward to show that $(d-2)\left(1+2^{-(e+d)}\right)^{d-2}\left(1-2^{-(e+d)}\right)^{-(d-2)} \leqslant 2^{d}$ for an integer $d \geqslant 3$. Hence $\left|\|\mathbf{x}\|^{-(d-2)}-\|\hat{\mathbf{x}}(\mathbf{x})\|^{-(d-2)}\right| \leqslant$ $2^{-e}\|\mathbf{x}\|^{-(d-2)}$.

First choose a fixed point $\mathbf{x}$ of satisfying $\|\mathbf{x}\|^{-(d-2)}=\|\mathcal{T}\|_{\sigma}$. Therefore $L(\mathcal{T}) \geqslant\left(1-2^{-e}\right)\|\mathcal{T}\|_{\sigma}$. Assume that $L(\mathcal{T})=\|\hat{\mathbf{x}}(\mathbf{x})\|^{-(d-2)}$ for some $\mathbf{x} \in X \backslash\{\mathbf{0}\}$. Then $L(\mathcal{T}) \leqslant\left(1+2^{-e}\right)\|\mathbf{x}\|^{-(d-2)} \leqslant\left(1+2^{-e}\right)\|\mathcal{T}\|_{\sigma}$. Thus $\left|\|\mathcal{T}\|_{\sigma}-L(\mathcal{T})\right| \leqslant 2^{-e}\|\mathcal{T}\|_{\sigma}$.

It is left to show that the bit complexity of computing $L(\mathcal{T})$ is $\tilde{\mathcal{O}}\left((\tau+e) d^{8 n}\right)$. This follows from the proof of Lemma 5 in Appendix 2. First note that in Lemma $5 m=2 n$. Next observe that the value of $\ell$ in Lemma 5 is $e+k$, where $k$ is given by (19).

We now present the main result of this section:

Theorem 13. Let $d \geqslant 3$ be an integer. Assume that $\mathcal{J} \in \mathrm{S}^{d} \mathbb{Z}[\mathbf{i}]^{n}$ is a strongly nonsingular tensor that satisfies $\|\mathcal{J}\| \leqslant 2^{c}, c \in \mathbb{N}$. Suppose that $\mathcal{T} \in \mathrm{S}^{d} \mathbb{Z}[\mathbf{i}]^{n}$, and each coordinate of $\mathcal{T}$ is bounded above by $2^{\tau}, \tau \in \mathbb{N}$. For a given $b, e \in \mathbb{N}$ we can compute $L(\mathcal{T})$ satisfying

$$
\left|\|\mathcal{T}\|_{\sigma}-L(\mathcal{T})\right| \leqslant 2^{-e}\|\mathcal{T}\|
$$

with probability greater than $1-(d-1)^{-2 n b}$. The bit complexity of computing $L(\mathcal{T})$ is $\tilde{O}\left(\left(\tau+c+2 n b \log _{2}(d-\right.\right.$ 1) $\left.+e) d^{8 n}\right)$.

Proof. Clearly, it is enough to assume that $\mathcal{T} \neq 0$. Lemma 11 yields that the set of strongly singular symmetric tensors is the zero set of $\Delta(\mathbf{u}) p(\mathbf{u}, \overline{\mathbf{u}})$, where $\mathbf{u} \in \mathbb{C}^{J(d, n)}$ represents the polynomial $f(\mathbf{x})=\mathcal{S} \times \otimes^{d} \mathbf{x}$, for $\mathcal{S} \in \mathrm{S}^{d} \mathbb{C}^{n}$. Here $\Delta(\mathbf{u})$ is a polynomial of degree $n(d-1)^{n-1}$, and $\Delta(\mathbf{u})=0$ is the hyperdeterminant variety. The polynomial $p(\mathbf{u}, \overline{\mathbf{u}})$ is a polynomial of degree $2\left((d-1)^{2 n}-1\right)$. Let $g(\mathbf{x})=\mathcal{J} \times \otimes^{d} \mathbf{x}$. Denote by $\mathbf{g} \in \mathbb{C}^{J(d, n)}$ the vector corresponding to $g(\mathbf{x})$. We now consider the affine line of polynomials $f_{t}(\mathbf{x})=t f(\mathbf{x})+g(\mathbf{x})$ for $t \in \mathbb{R}$. The value of the polynomial $\Delta p$ on $t \mathbf{f}+\mathbf{g}$ is $q(t)=\Delta(t \mathbf{f}+\mathbf{g}) p(t \mathbf{f}+\mathbf{g}, t \overline{\mathbf{f}}+\overline{\mathbf{g}})$. As $\mathcal{J}$ is strongly nonsingular $q(0) \neq 0$. Hence $q(t)$ is a nonzero polynomial of degree at most $n(d-1)^{n-1}+2\left((d-1)^{2 n}-1\right)<3(d-1)^{2 n}$.

Let $A=\{M, M+2, \ldots, M+N-1\} \subset \mathbb{Z}$. We assume that $M=2^{c+e+2}$ and $N=3(d-1)^{2 n(b+1)}$. The cardinality of $A$ is $N$. For each $a \in A$, let us consider the tensor $\mathcal{T}(a)=a \mathcal{T}+\mathcal{J}$. Note $q(t)$ vanishes at most at $n(d-1)^{n-1}+2\left((d-1)^{2 n}-1\right)$ points of $A$. Choose a random $a \in A$ from the uniform distribution on $\mathrm{A}$. Then with probability greater than $1-(d-1)^{-2 n b}$ the tensor $\mathcal{T}(a)$ is strongly nonsingular.

Let $L(\mathcal{T}(a))$ be the approximation given by Theorem 12, where we replace $e$ by $e+2$. We now choose $L(\mathcal{T})=\frac{1}{a} L(\mathcal{T}(a))$. We claim that $\left|\|\mathcal{T}\|_{\sigma}-L(\mathcal{T})\right| \leqslant 2^{-e}\|\mathcal{T}\|$. Indeed, the inequality of Theorem 12 yields

$$
\left|\left\|\mathcal{T}+\frac{1}{a} \mathcal{J}\right\|_{\sigma}-L(\mathcal{T})\right| \leqslant \frac{2^{-e}}{4}\left\|\mathcal{T}+\frac{1}{a} \mathcal{J}\right\|_{\sigma} .
$$


As $\mathcal{T} \in \mathrm{S}^{d} \mathbb{Z}[\mathbf{i}] \backslash\{0\}$ it follows that $\|\mathcal{T}\| \geqslant 1$. Since $a \geqslant 2^{c+e+2}$ and $\|\mathcal{J}\| \leqslant 2^{c}$ we deduce

$$
\begin{aligned}
& \left|\|\mathcal{T}\|_{\sigma}-L(\mathcal{T})\right| \leqslant\left|\left\|\mathcal{T}+\frac{1}{a} \mathcal{J}\right\|_{\sigma}-L(\mathcal{T})\|+\mid\| \mathcal{T}\left\|_{\sigma}-\right\| \mathcal{T}+\frac{1}{a} \mathcal{J}\left\|_{\sigma}\right\| \leqslant\right. \\
& 2^{-(e+2)}\left\|\mathcal{T}+\frac{1}{a} \mathcal{J}\right\|_{\sigma}+\frac{1}{a}\|\mathcal{J}\|_{\sigma} \leqslant 2^{-(e+2)}\|\mathcal{T}\|_{\sigma}+\left(2^{-(e+2)}+1\right) \frac{1}{a}\|\mathcal{J}\|_{\sigma} \leqslant \\
& 2^{-(e+2)}\left(\|\mathcal{T}\|_{\sigma}+2^{-(e+2)}+1\right) \leqslant 2^{-(e+2)}\left(\|\mathcal{T}\|+\left(2^{-(e+2)}+1\right)\|\mathcal{T}\|\right)<2^{-e}\|\mathcal{T}\| .
\end{aligned}
$$

It is left to show that the bit complexity of finding $L(\mathcal{T})$ is $\tilde{O}\left(\left(\tau+c+2 n b \log _{2}(d-1)+c\right) d^{8 n}\right)$. This follows from Theorem 12, Indeed observe that each entry by $\mathcal{T}(a)$ is bounded by $2^{\tau+c+2}(d-1)^{2 n(b+1)}$.

We remark that we can find a strongly nonsingular $\mathcal{J} \in \mathrm{S}^{d} \mathbb{Z}^{n}$ as follows: We choose a tensor of the form given in the proof of Lemma 11 by choosing $a_{2}, \ldots, a_{n} \in \mathbb{Z}$ at random.

We now give a similar complexity result for an approximation of $\|\mathcal{T}\|_{\sigma, \mathbb{R}}$ :

Theorem 14. Let $d \geqslant 3$ be an integer. Suppose that $\mathcal{T} \in \mathrm{S}^{d} \mathbb{Z}^{n}$, and each coordinate of $\mathcal{T}$ is bounded above by $2^{\tau}, \tau \in \mathbb{N}$. For a given $e \in \mathbb{N}$ we can compute $L(\mathcal{T})$ that satisfies the following conditions:

(1) Assume that $\mathcal{T}$ is strongly nonsingular. Then $\left|\|\mathcal{T}\|_{\sigma, \mathbb{R}}-L(\mathcal{T})\right| \leqslant 2^{-e}\|\mathcal{T}\|_{\sigma, \mathbb{R}}$. The bit complexity of computing $L(\mathcal{T})$ is $\tilde{\mathcal{O}}\left((\tau+e) d^{4 n}\right)$.

(2) Assume that $\mathcal{J} \in \mathrm{S}^{d} \mathbb{Z}^{n}$ is a strongly nonsingular tensor that satisfies $\|\mathcal{J}\| \leqslant 2^{c}, c \in \mathbb{N}$. For a given $b \in \mathbb{N}$ we can compute $L(\mathcal{T})$ with probability greater than $1-(d-1)^{-2 n b}$ that satisfies $\mid\|\mathcal{T}\|_{\sigma, \mathbb{R}}-$ $L(\mathcal{T}) \mid \leqslant 2^{-e}\|\mathcal{T}\|$. The bit complexity of computing $L(\mathcal{T})$ is $\tilde{O}\left(\left(\tau+c+2 n b \log _{2}(d-1)+e\right) d^{4 n}\right)$. With probability

Proof. We point out briefly the corresponding modifications of the proofs of Theorems 12 and 13 respectively. Let $f(\mathbf{x})=\mathcal{T} \times \otimes^{d} \mathbf{x}$. First observe that $\overline{\mathbf{F}}=\mathbf{F}$. Hence any fixed point of $\mathbf{F}$ is a fixed point $\mathbf{H}$.

First assume that $\mathcal{T}$ is nonsingular. Then $x_{1}$ coordinates of $(d-1)^{n}$ fixed points of $\mathbf{F}$ are pairwise distinct. We now find the Gröbner basis of the system $\mathbf{F}(\mathbf{x})-\mathbf{x}=\mathbf{0}$ with respect to the order $x_{1} \prec \cdots \prec x_{n}$. It is of the form $p_{1}\left(x_{1}\right), x_{2}-p_{2}\left(x_{1}\right), \ldots, x_{n}-p_{n}\left(x_{1}\right)$, where $p_{1}, \ldots, p_{n} \in \mathbb{R}\left[x_{1}\right]$. Here $\operatorname{deg} p_{1}=D$, where $D=(d-1)^{n}$. Furthermore $p_{1}\left(x_{1}\right)$ has $D$ distinct roots. Recall that $\operatorname{deg} p_{i}<D$ for $i>1$. The set of the fixed points of $\mathbf{F}$ is given by (17).

Recall that $\|\mathcal{T}\|_{\sigma, \mathbb{R}}$ can be computed using (12). Thus we need to determine fix $\mathbb{R}_{\mathbb{R}}(\mathbf{F})$ with precision $2^{e+k}$, as in the proof of Theorem 12. This can be done as follows. First consider $X_{\mathbb{R}}=X \cap \mathbb{R}^{n}$. For each approximation of a root of $x_{1}$ with precision $2^{e+k}$, we check if the disk $\left|z-x_{1}\right|<2^{e+k}$ contains a real root of $p_{1}\left(x_{1}\right)$. If yes, we replace $x_{1}$ by $\tilde{x}_{1} \in \mathbb{R}$. Then a real approximation of the real fixed point is $\left(\tilde{x}_{1}, p_{2}\left(\tilde{x}_{1}\right), \ldots, p_{n}\left(\tilde{x}_{n}\right)\right)^{\top}$. Similarly we can find a real approximation of $\bar{\omega}_{d-2} X \cap \mathbb{R}^{n}$ for an even $n$. (See Theorem 7) Now we proceed as in the proof of Theorem 12

For a general $\mathcal{T} \in \mathrm{S}^{d} \mathbb{Z}^{n}$ we repeat the arguments of the proof of Theorem 13 taking into account the above results for a strongly nonsingular $\mathcal{T} \in \mathrm{S}^{d} \mathbb{Z}^{n}$.

We conclude this section with the following NP-hardness result for an arbitrary approximation of the spectral norm of a real or complex valued homogeneous quartic polynomial in $n$ variables.

Theorem 15. Let $A=\left[A_{i, j}\right]$ be an $n \times n$ nonzero symmetric matrix with $\{0,1\}$ entries and zero diagonal. Set

$$
f_{A}=\sum_{i=j=1}^{n} A_{i, j} x_{i}^{2} x_{j}^{2} .
$$

(1) Let $2 e$ be the number of nonzero elements in $A$. Then

$$
\left\|\mathbf{f}_{A}\right\|=\frac{\sqrt{2 e}}{\sqrt{3}}, \quad \frac{\sqrt{2}}{\sqrt{3}} \leqslant\left\|\mathbf{f}_{A}\right\| \leqslant \frac{\sqrt{n(n-1)}}{\sqrt{3}}<n,
$$

where $\left\|\mathbf{f}_{A}\right\|$ is the norm defined in Lemma 1 .

(2) Equality

$$
\left\|f_{A}\right\|_{\sigma, \mathbb{R}}=\left\|f_{A}\right\|_{\sigma}=1-1 / \kappa(A)
$$

holds, where $\kappa(A)$ is an integer in the set $[n]$. 
(3) It is NP-hard to compute an approximation of $\left\|f_{A}\right\|_{\sigma}$ within relative precision $\delta<\frac{1}{2 n^{2}(n+1)}$ with respect to $\left\|\mathbf{f}_{A}\right\|$.

Proof. (1) Assume that $f_{A}$ corresponds to $\mathcal{S}=\left[\mathcal{S}_{k_{1}, k_{2}, k_{3}, k_{4}}\right] \in \mathrm{S}^{4} \mathbb{C}^{n}$. Then $\mathcal{S}_{k_{1}, k_{2}, k_{3}, k_{4}}=1 / 3$ if the multiset $\left\{k_{1}, k_{2}, k_{3}, k_{4}\right\}$ is of the form $\{i, i, j, j\}, i<j$ and $A_{i, j}=A_{j, i}=1$. (The number of nonzero $\mathcal{S}_{k_{1}, k_{2}, k_{3}, k_{4}}$ corresponding to $\{i, i, j, j\}$ is $4 ! /(2 !)^{2}=6$.) Otherwise $\mathcal{S}_{k_{1}, k_{2}, k_{3}, k_{4}}=0$. Hence $\left\|\mathbf{f}_{A}\right\|=\|\mathcal{S}\|=\sqrt{2 e} / \sqrt{3}$. The second inequality follows straightforward.

(2) The matrix $A$ is the adjacency matrix of the following simple undirected graph $G=(V, E)$ : Here $V=[n]$ and the edge $(i, j)$ is in $E$ if and only if $A_{i, j}=1$. Let $\kappa(A)$ be the cardinality of the maximal clique in $G$, (the clique number of $G$ ). The equality $\left\|f_{A}\right\|_{\sigma, \mathbb{R}}=1-1 / \kappa(A)$ is [26, (8.2)]. As $\mathcal{S}$ has nonnegative entries it follows that $\left\|f_{A}\right\|_{\sigma, \mathbb{R}}=\left\|f_{A}\right\|_{\sigma}[26]$.

(3) Since $\left\|f_{A}\right\|_{\sigma}=1-1 / \kappa(A)$, an approximation of $\left\|f_{A}\right\|_{\sigma}$ within relative precision $\varepsilon<\frac{1}{2 n^{2}(n+1)}$ with respect to $\left\|\mathbf{f}_{A}\right\|$ determines the clique number. However, it is an NP-complete problem to determine the clique number of a simple graph [40.

\section{Polynomial-time Computability of SPeCtral norm of Symmetric $d$-Qubits}

In this section we improve the results of the previous section for the case $n=2$. We parametrize $\mathcal{S}=\left[\mathcal{S}_{i_{1}, \ldots, i_{d}}\right] \in \mathrm{S}^{d} \mathbb{F}^{2}$ by $\mathbf{s}=\left(s_{0}, \ldots, s_{d}\right)$ as follows: $\mathcal{S}_{i_{1}, \ldots, i_{d}}=s_{k}$ if exactly $k$ indices from the multiset

$\left\{i_{1}, \ldots, i_{d}\right\}$ are equal to 2 . Note that exactly $\left(\begin{array}{l}d \\ k\end{array}\right)$ entries of $\mathcal{S}$ are equal to $s_{k}$. Hence $\|\mathcal{S}\|=\sqrt{\sum_{k=0}^{d}\left(\begin{array}{l}d \\ k\end{array}\right)\left|s_{k}\right|^{2}}$.

The following lemma is a restatement of some results of Lemma 1 .

Lemma 16. Let $\mathcal{S} \in \mathrm{S}^{d} \mathbb{C}^{2}$ and associate with $\mathcal{S}$ the vector $\mathbf{s}=\left(s_{0}, \ldots, s_{d}\right)^{\top} \in \mathbb{C}^{d+1}$. Denote

$$
\phi(z)=\sum_{j=0}^{d}\left(\begin{array}{l}
d \\
j
\end{array}\right) s_{j} z^{j}
$$

Then

(1) Let $f(\mathbf{x})=\mathcal{S} \times \otimes^{d} \mathbf{x}$ and $\mathcal{S} \times \otimes^{d-1} \mathbf{x}=\mathbf{F}(\mathbf{x})=\left(F_{1}(\mathbf{x}), F_{2}(\mathbf{x})\right)^{\top}$, where $\mathbf{x}=\left(x_{1}, x_{2}\right)^{\top}$. Then

$$
\begin{aligned}
& f(\mathbf{x})=\sum_{j=0}^{d}\left(\begin{array}{l}
d \\
j
\end{array}\right) s_{j} x_{1}^{d-j} x_{2}^{j}=x_{1}^{d} \phi\left(\frac{x_{2}}{x_{1}}\right), \\
& F_{1}(\mathbf{x})=\sum_{j=0}^{d-1}\left(\begin{array}{c}
d-1 \\
j
\end{array}\right) s_{j} x_{1}^{d-1-j} x_{2}^{j}=\frac{1}{d} \frac{\partial f}{\partial x_{1}}, F_{2}(\mathbf{x})=\sum_{j=0}^{d-1}\left(\begin{array}{c}
d-1 \\
j
\end{array}\right) s_{j+1} x_{1}^{d-j-1} x_{2}^{j}=\frac{1}{d} \frac{\partial f}{\partial x_{2}} .
\end{aligned}
$$

(2) For $\mathbf{s}=\left(0, \ldots, 0, s_{d}\right)^{\top}$ we have $\|\mathcal{S}\|_{\sigma, \mathbb{F}}=\left|s_{d}\right|$.

Proof. (1). Use equalities (4) and (5).

(2). Assume that $\mathbf{s}=\left(0, \ldots, 0, s_{d}\right)^{\top}$. Then $\mathcal{S} \times \otimes^{d} \mathbf{x}=s_{d} x_{2}^{d}$. Hence $\|\mathcal{S}\|_{\sigma, \mathbb{F}}=\left|s_{d}\right|$.

The next proposition studies the fixed points of $\mathbf{F}$ and $\mathbf{H}$ for the case $n=2$.

Proposition 17. Suppose that the assumptions and notations of Lemma [16 hold. Assume that $d \geqslant 3$ and

$$
\mathbf{s} \neq\left(0, \ldots, 0, s_{d}\right)^{\top} .
$$

(1) Define polynomials $p(z), q(z)$ and the rational function $r(z)$ as follows

$$
p(z)=\sum_{j=0}^{d-1}\left(\begin{array}{c}
d-1 \\
j
\end{array}\right) s_{j+1} z^{j}, q(z)=\sum_{j=0}^{d-1}\left(\begin{array}{c}
d-1 \\
j
\end{array}\right) s_{j} z^{j}, r(z)=\frac{p(z)}{q(z)} .
$$

Then

$$
p(z)=\frac{1}{d} \phi^{\prime}(z), q(z)=\phi(z)-\frac{1}{d} z \phi^{\prime}(z), r(z)=\frac{\phi^{\prime}(z)}{d \phi(z)-z \phi^{\prime}(z)} .
$$


(2) Suppose that $\mathbf{F}(\mathbf{x})=\left(x_{1}, x_{2}\right)^{\top} \neq \mathbf{0}$. First assume that $x_{1} \neq 0$. Let $z=x_{2} / x_{1}$. Then

$$
z q(z)-p(z)=0 \text { and } q(z) \neq 0 \text {. }
$$

Vice versa, for each $z \in \mathbb{C}$ satisfying the above conditions there are exactly $d-2$ fixed points of $\mathbf{F}$ of the form $\left(x_{1}, x_{1} z\right)$, where $x_{1}$ satisfies $x_{1}^{d-2}=\frac{1}{q(z)}$.

Second assume that $x_{1}=0$. Then $s_{d-1}=0$ and $s_{d} \neq 0$. Vice versa, if $s_{d-1}=0$ and $s_{d} \neq 0$ then there are exactly $d-2$ nonzero fixed points of the form $\left(0, x_{2}\right)$, where $x_{2}$ satisfies $x_{2}^{d-2}=1 / s_{d}$.

(3) Suppose that $\mathbf{F}(\mathbf{x})=\left(\bar{x}_{1}, \bar{x}_{2}\right) \neq \mathbf{0}$. First assume that $x_{1} \neq 0$. Let $z=x_{2} / x_{1}$. Then

$$
\bar{z} q(z)-p(z)=0
$$

and $q(z) \neq 0$. Vice versa, for each $z \in \mathbb{C}$ satisfying the above conditions there are exactly $d$ antifixed points of $\mathbf{F}$ of the form $\left(x_{1}, x_{1} z\right)$, where $x_{1}$ satisfies $x_{1}=\frac{\zeta}{|q(z)|^{1 /(d-2)}}$ and $\zeta^{d}=1$.

Second assume that $x_{1}=0$. Then $s_{d-1}=0$ and $s_{d} \neq 0$. Vice versa, if $s_{d-1}=0$ and $s_{d} \neq 0$ then there are $d$ nonzero fixed points of the form $\left(0, \frac{\zeta}{\left|s_{d}\right|^{1 /(d-2)}}\right)$ and $\zeta^{d}=1$.

(4) Let

$$
\bar{p}(z)=\sum_{j=0}^{d-1}\left(\begin{array}{c}
d-1 \\
j
\end{array}\right) \bar{s}_{j+1} z^{j}, \bar{q}(z)=\sum_{j=0}^{d-1}\left(\begin{array}{c}
d-1 \\
j
\end{array}\right) \bar{s}_{j} z^{j}, \bar{r}(z)=\frac{\bar{p}(z)}{\bar{q}(z)}, g(z)=\bar{r}(r(z)) .
$$

Then $g(z)=\frac{u(z)}{v(z)}$, where

$$
\begin{aligned}
& u(z)=\sum_{j=0}^{d-1}\left(\begin{array}{c}
d-1 \\
j
\end{array}\right) \bar{s}_{j+1}\left(\sum_{k=0}^{d-1}\left(\begin{array}{c}
d-1 \\
k
\end{array}\right) s_{k+1} z^{k}\right)^{j}\left(\sum_{k=0}^{d-1}\left(\begin{array}{c}
d-1 \\
k
\end{array}\right) s_{k} z^{k}\right)^{d-1-j} \\
& v(z)=\sum_{j=0}^{d-1}\left(\begin{array}{c}
d-1 \\
j
\end{array}\right) \bar{s}_{j}\left(\sum_{k=0}^{d-1}\left(\begin{array}{c}
d-1 \\
k
\end{array}\right) s_{k+1} z^{k}\right)^{j}\left(\sum_{k=0}^{d-1}\left(\begin{array}{c}
d-1 \\
k
\end{array}\right) s_{k} z^{k}\right)^{d-1-j} .
\end{aligned}
$$

Suppose that $\mathbf{F}(\mathbf{x})=\left(\bar{x}_{1}, \bar{x}_{2}\right)^{\top} \neq \mathbf{0}$ as in (3). Assume that $x_{1} \neq 0$. Then each solution of (24) satisfies

$$
z v(z)-u(z)=0
$$

and $v(z) \neq 0$. This is a polynomial equation of degree at most $(d-1)^{2}+1$.

Proof. (1) The assumption $\mathbf{s} \neq\left(0, \ldots, 0, s_{d}\right)^{\top}$ is equivalent to the assumption that the polynomial $q(z)$ is not zero identically. Assume that $x_{1} \neq 0$. Let $z=\frac{x_{2}}{x_{1}}$. Lemma 16 yields: $\frac{F_{2}\left(x_{1}, x_{1}\right)}{x_{1}^{d-1}}=p(z)$ and $\frac{F_{1}\left(x_{1}, x_{1}\right)}{x_{1}^{d-1}}=q(z)$. Recall that

$$
\begin{array}{r}
d F_{2}=\frac{\partial f}{\partial x_{2}}=\frac{\partial\left(x_{1}^{d} \phi\left(\frac{x_{2}}{x_{1}}\right)\right)}{\partial x_{2}}=x_{1}^{d-1} \phi^{\prime}\left(\frac{x_{2}}{x_{1}}\right), \\
d F_{1}=\frac{\partial f}{\partial x_{1}}=\frac{\partial\left(x_{1}^{d} \phi\left(\frac{x_{2}}{x_{1}}\right)\right)}{\partial x_{1}}=d x_{1}^{d-1} \phi\left(\frac{x_{2}}{x_{1}}\right)-x_{2} x_{1}^{d-2} \phi^{\prime}\left(\frac{x_{2}}{x_{1}}\right) .
\end{array}
$$

These equalities yield (22).

(2) Assume that $\mathbf{F}(\mathbf{x})=\left(x_{1}, x_{2}\right)^{\top} \neq \mathbf{0}$. Suppose first that $x_{1} \neq 0$. Then $x_{1}^{d-1} q(z)=F_{1}(\mathbf{x})=x_{1}$ and $x_{1}^{d-1} p(z)=F_{2}(\mathbf{x})=x_{2}$. As $x_{1} \neq 0$ it follows that $q(z) \neq 0$. Divide the second equality by the first one to deduce (23). Note that $x_{1}^{d-2}=\frac{1}{q(z)}$.

Assume that $z \in \mathbb{C}$ satisfies (23). Suppose furthermore that $x_{1}^{d-2}=\frac{1}{q(z)}$. Let $\mathbf{x}=\left(x_{1}, x_{1} z\right)^{\top}$. Then

$$
F_{1}(\mathbf{x})=x_{1}^{d-1} q(z)=x_{1}, \quad F_{2}(\mathbf{x})=x_{1}^{d-1} p(z)=x_{1}^{d-1} z q(z)=x_{1}^{d-1}\left(x_{2} / x_{1}\right) q(z)=x_{1}^{d-2} q(z) x_{2}=x_{2} .
$$

Hence each $z$ that satisfies (23) gives rise to exactly $d-2$ distinct nonzero fixed points of $\mathbf{F}$.

Assume that $x_{1}=0$. Hence $x_{2} \neq 0$. Note that $F_{1}(\mathbf{x})=s_{d-1} x_{2}^{d-1}$. As $F_{1}(\mathbf{x})=x_{0}=0$ we deduce that $s_{d-1}=0$. Clearly, $F_{2}(\mathbf{x})=s_{d} x_{2}^{d-1}=x_{2}$. As $x_{2} \neq 0$ it follows that $s_{d} \neq 0$.

Suppose that $s_{d-1}=0$ and $s_{d} \neq 0$. Then all nonzero fixed points of $\mathbf{F}$ of the form $\left(0, x_{2}\right)^{\top}$ are exactly those $x_{2}$ satisfying $x_{2}^{d-2}=1 / s_{d}$. 
(3) The proof of this part is very similar to the part (2) and we leave it to the reader.

(4) From the definitions of $p(z), q(z), \bar{p}(z), \bar{q}(z), \bar{r}(z)$ and $g(z)$ we deduce straightforward the identities (25)) and (26). Let

$$
u(z)=\sum_{k=0}^{(d-1)^{2}} u_{k} z^{k}, \quad v(z)=\sum_{k=0}^{(d-1)^{2}} v_{k} z^{k} .
$$

Then

$$
u_{(d-1)^{2}}=\sum_{j=0}^{d-1}\left(\begin{array}{c}
d-1 \\
j
\end{array}\right) \bar{s}_{j+1} s_{d}^{j} s_{d-1}^{d-1-j}, v_{(d-1)^{2}}=\sum_{j=0}^{d-1}\left(\begin{array}{c}
d-1 \\
j
\end{array}\right) \bar{s}_{j} s_{d}^{j} s_{d-1}^{d-1-j} .
$$

Hence the polynomial $z v(z)-u(z)$ is of degree at most $(d-1)^{2}+1$. Suppose $\mathbf{x}=\left(x_{1}, x_{2}\right)^{\top} \in \operatorname{fix}(\mathbf{H}), x_{1} \neq 0$ : $\overline{\mathbf{F}}(\mathbf{F}(\mathbf{x}))=\mathbf{x}$. Since we assumed that $x_{1} \neq 0$ it follows that

$$
u(z)=\frac{\overline{\mathbf{F}}_{2}(\mathbf{F}(\mathbf{x}))}{x_{1}^{(d-1)^{2}}}, \quad v(z)=\frac{\overline{\mathbf{F}}_{1}(\mathbf{F}(\mathbf{x}))}{x_{1}^{(d-1)^{2}}}=x_{1}^{-(d-1)^{2}+1} \neq 0 .
$$

Therefore

$$
\bar{r}(r(z))=\frac{\overline{\mathbf{F}}_{2}(\mathbf{F}(\mathbf{x})) x_{1}^{-(d-1)^{2}}}{\overline{\mathbf{F}}_{1}(\mathbf{F}(\mathbf{x})) x_{1}^{-(d-1)^{2}}}=\frac{x_{2}}{x_{1}}=z .
$$

This yields (27), which is a polynomial equation of degree at most $(d-1)^{2}+1$.

The following theorem gives much more efficient way to compute the spectral norm of symmetric qubits than the general methods suggested in $\$ 5$.

Theorem 18. Let $\mathcal{S} \in \mathrm{S}^{d} \mathbb{C}^{2}, d>2$ and associate with $\mathcal{S}$ the vector $\mathbf{s}=\left(s_{0}, \ldots, s_{d}\right)^{\top} \in \mathbb{C}^{d+1}$. Let $\phi(z)$ be given by (20). Assume the notations of Proposition 17. Then

(1) The polynomial $z v(z)-u(z)$ is a zero polynomial if and only if one of the following conditions hold. Either

$$
\mathbf{s}=A\left(\delta_{1(k+1)}, \ldots, \delta_{(d+1)(k+1)}\right)^{\top} \text { for } k \in[d-1],\left(f(\mathbf{x})=A\left(\begin{array}{l}
d \\
k
\end{array}\right) x_{1}^{d-k} x_{2}^{k}\right),
$$

where $A$ is a nonzero scalar constant and $\delta_{i j}$ is Kronecker's delta function. For this $\mathcal{S} \in \mathrm{S}^{d} \mathbb{F}^{2}$ we have

Or $\mathcal{S}$ has corresponding $\phi$ given by

$$
\|\mathcal{S}\|_{\sigma, \mathbb{F}}=|A|\left(\begin{array}{l}
d \\
k
\end{array}\right)\left(1-\frac{k}{d}\right)^{\frac{d-k}{2}}\left(\frac{k}{d}\right)^{\frac{k}{2}} .
$$

$$
\begin{aligned}
& \phi(z)=A(z+a)^{p}(z+b)^{d-p}, \quad A \neq 0, \\
& a=e^{-\theta \mathbf{i}} c, b=-e^{-\theta \mathbf{i}} c^{-1}, c \in \mathbb{R} \backslash\{0\}, \theta \in \mathbb{R}, p \in[d-1] .
\end{aligned}
$$

Assume that $\mathcal{S} \in \mathrm{S}^{d} \mathbb{C}^{2}$ corresponds to $\phi$ is of the form (29). Then $\|\mathcal{S}\|_{\sigma}$ can be computed to an

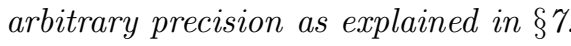

(2) Let

$$
R_{1}=\{z \in \mathbb{C}, z v(z)-u(z)=0\}, \quad R_{1}^{\prime}=R_{1} \cap \mathbb{R} .
$$

Suppose that $\mathcal{S} \in \mathrm{S}^{d} \mathbb{C}^{2}$ is not of the form given in (1). (Hence the set $R_{1}$ is finite.) Then $\|\mathcal{S}\|_{\sigma}$ has the following maximum characterization:

$$
\|\mathcal{S}\|_{\sigma}=\max \left\{\left|s_{d}\right|, \max \left\{\frac{|\phi(z)|}{\left(1+|z|^{2}\right)^{\frac{d}{2}}}, z \in R_{1}\right\}\right\} .
$$

(3) Assume that $\mathcal{S} \in \mathrm{S}^{d} \mathbb{R}^{2}$. Let

$$
R=\{z \in \mathbb{C}, z q(z)-p(z)=0\}, \quad R^{\prime}=R \cap \mathbb{R}
$$

Then $z q(z)-p(z)$ is a zero polynomial if and only if $d$ is even and $\phi(z)=A\left(z^{2}+1\right)^{d / 2}$. In this case $\|\mathcal{S}\|_{\sigma, \mathbb{R}}=|A|$. 
Assume that $\phi(z)$ is not of the form $A\left(z^{2}+1\right)^{d / 2}$. Then $\|\mathcal{S}\|_{\sigma, \mathbb{R}}$ has the following maximum characterizations:

$$
\|\mathcal{S}\|_{\sigma, \mathbb{R}}=\max \left\{\left|s_{d}\right|, \max \left\{\frac{|\phi(z)|}{\left(1+|z|^{2}\right)^{\frac{d}{2}}}, z \in R^{\prime}\right\}\right\} .
$$

Proof. (1) The analysis of the exceptional cases is given in $\$ 7$.

(2) Assume that $\mathbf{s}=\left(s_{0}, \ldots, s_{d}\right)^{\top} \in \mathbb{C}^{d+1}$ is the vector corresponding to $\mathcal{S}=\left[\mathcal{S}_{i_{1}, \ldots, i_{d}}\right] \in \mathrm{S}^{d} \mathbb{C}^{2}$. Let $\omega$ be the righthand side of (30). We claim that Banach's characterization (77) yields that $\omega \leqslant\|\mathcal{S}\|_{\sigma}$. Indeed, $\|\mathcal{S}\|_{\sigma} \geqslant\left|\mathcal{S} \times\left(\otimes^{d}(0,1)^{\top}\right)\right|=\left|\mathcal{S}_{2, \ldots, 2}\right|=\left|s_{d}\right|$. Assume now that $\mathbf{x}=\left(x_{1}, x_{2}\right)^{\top}$ and $x_{1} \neq 0$. Let $z=x_{2} / x_{1}$. Then $\left|\mathcal{S} \times\left(\otimes^{d} \mathbf{x}\right)\right| /\|\mathbf{x}\|^{d}=|\phi(z)| /\left(1+|z|^{2}\right)^{d / 2}$. Hence $\omega \leqslant\|\mathcal{S}\|_{\sigma}$. As any maximal point of $\left|\mathcal{S} \times\left(\otimes^{d} \mathbf{x}\right)\right|$ on $\mathrm{S}(2, \mathbb{C})$ is either $\zeta(0,1)^{\top},|\zeta|=1$, or of the form $\zeta(1, z), \zeta \in \mathbb{C} \backslash\{0\}$, where $z \in R_{1}$ we deduce that $\omega=\|\mathcal{S}\|_{\sigma}$. (3) Assume that $z q(z)-p(z)$ is identically zero. The equalities (22) yield that

$$
z \phi=(1 / d)\left(z^{2}+1\right) \phi^{\prime}(z) \Rightarrow(d / 2)\left(\ln \left(z^{2}+1\right)\right)^{\prime}=(\ln \phi)^{\prime} \Rightarrow \phi=A\left(z^{2}+1\right)^{d / 2} .
$$

As $\phi$ is a polynomial, it follows that $d$ is even. Note that $\mathcal{S} \times\left(\otimes^{d} \mathbf{x}\right)=A\left(x_{0}^{2}+x_{1}^{2}\right)^{d / 2}$. Hence $\|\mathcal{S}\|_{\sigma, \mathbb{R}}=|A|$.

Assume now $\phi$ is not of the form $A\left(z^{2}+1\right)^{d / 2}$. Then the arguments of the proof of part (2) yield the equality (31).

We now give the complexity of finding the spectral norm of $\mathcal{S} \in \mathrm{S}^{d} \mathbb{Z}[\mathbf{i}]^{2}$ or $\mathcal{S} \in \mathrm{S}^{d} \mathbb{Z}^{2}$.

Theorem 19. Let $\mathcal{S} \in \mathrm{S}^{d} \mathbb{Z}[\mathbf{i}]^{2}, d>2$ and associate with $\mathcal{S}$ the vector $\mathbf{s}=\left(s_{0}, \ldots, s_{d}\right)^{\top} \in \mathbb{Z}[\mathbf{i}]^{d+1}$. Assume that $\left|s_{i-1}\right| \leqslant 2^{\tau}, i \in[d+1]$ for some $\tau \in \mathbb{N}$. Let $\phi(z)$ be given by (20). Assume the notations of Proposition 17. For a given $e \in \mathbb{N}$ we can compute a rational $L(\mathcal{S})$ satisfying

$$
\left|\|\mathcal{S}\|_{\sigma, \mathbb{F}}-L(\mathcal{S})\right| \leqslant 2^{-e}\|\mathcal{S}\|_{\sigma, \mathbb{F}}
$$

under the following conditions:

(1) The tensor $\mathcal{S} \in \mathrm{S}^{d} \mathbb{Z}[\mathbf{i}]^{2}$ does not satisfy condition (1) of Theorem 18, The bit complexity of computation of $L(\mathcal{S})$ for $\mathbb{F}=\mathbb{C}$ is $\tilde{\mathcal{O}}\left(d^{2}\left(d^{4} \max \left(d^{2}, \tau\right)+e\right)\right)$.

(2) The tensor $\mathcal{S}$ has real integer entries. The bit complexity of computation of $L(\mathcal{S})$ for $\mathbb{F}=\mathbb{R}$ is $\hat{\mathcal{O}}\left(d\left(d^{2} \max (d, \tau)+e\right)\right)$.

Proof. (1) The assumption that condition (1) of Theorem 18 does not hold is equivalent to the assumption that the polynomial $h(z)=z v(z)-u(z)$ is a nontrivial polynomial, whose degree $D$ satisfies $D \leqslant(d-1)^{2}+1<$ $d^{2}$. (See part (4) of Proposition [17,) Observe that $h(z)=z v(z)-u(z) \in(\mathbb{Z}[\mathbf{i}])[z]$, and its coefficients are bounded by $2^{2 \tau+2 d}$. As in the proof of Theorem 12, we approximate the roots of $h(z)$ with precision $2^{-(e+k)}$, where $k \in \mathbb{N}$ is specified later. Now we repeat the arguments of Theorem 12 by replacing the characterization (18) with (30). This yields the estimate $\left|\|\mathcal{S}\|_{\sigma}-L(\mathcal{S})\right| \leqslant 2^{-e}\|\mathcal{S}\|_{\sigma}$. The bit complexity estimate $\tilde{\mathcal{O}}\left(d^{2}\left(d^{4} \max \left(d^{2}, \tau\right)+e\right)\right)$ follows from [46]. (See Appendix 2.)

(2) We now repeat the arguments of the proof of (1) with the following modifications. Assume that $\phi(z)$ corresponds to $\mathcal{S} \in \mathrm{S}^{d} \mathbb{Z}^{n}$. Suppose first $\phi(z)=A\left(z^{2}+1\right)^{d / 2}$, where $d$ is even. Then $\|\mathcal{S}\|_{\sigma, \mathbb{R}}=|A|$ and let $L(\mathcal{S})=|A|$. Assume now that $\phi(z) \neq A\left(z^{2}+1\right)^{d / 2}$. Then $h(z)=z q(z)-p(z)$ is a nonzero polynomial of degree $D \leqslant d$. We approximate the roots of $h(z)$ to approximate the set $R^{\prime}$ as given in part (3) of Theorem 18. We use characterization (31) to compute $L(\mathcal{S})$.

\section{The EXCEPtional CASES}

7.1. Analysis of the exceptional cases. In this subsection we discuss part (1) of Theorem 18, Assume that $g(z)=\bar{r}(r(z))=z$ identically. Recall that $r(z)$ can be viewed as a holomorphic map of the Riemann sphere. The degree of this map is $\delta \in \mathbb{N}$ since $g$ is not a constant map. Hence the degree of the map $g$ is $\delta^{2}$. Since $g$ is the identity map, its degree is 1 , it follows that $\delta=1$ and $r(z)$ is a Möbius map:

$$
r(z)=\frac{a z+b}{c z+d}, \quad a d-b c \neq 0 .
$$

Use the formula for $r(z)$ in (22) to deduce

$$
\frac{1}{d}(\log \phi(z))^{\prime}=\frac{1}{d} \frac{\phi^{\prime}(z)}{\phi(z)}=\frac{a z+b}{c z+d+z(a z+b)} .
$$


Let $l$ be the number of distinct roots of $\phi(z)$. Then the logarithmic derivative of $\phi(z)$ has exactly $l$ distinct poles. Comparing that with the above formula of the logarithmic derivative of $\phi$, we deduce that $\phi$ has either one (possibly) multiple root or two distinct roots.

First assume that $\phi(z)$ has one root of multiplicity $k: \phi(z)=A(z+a)^{k}$ for $k \in[d]$. Then

$$
r(z)=\frac{k}{(d-k) z+d a}, \quad \bar{r}(z)=\frac{k}{(d-k) z+d \bar{a}} .
$$

In this case $g(z) \equiv z$ if and only if $k \in[d-1]$ and $a=0$. Clearly, if $\phi(z)=A z^{k}$, where $A \neq 0$, then $g(z) \equiv z$. In this case $\mathcal{S} \times \otimes^{d} \mathbf{x}=A\left(\begin{array}{l}d \\ k\end{array}\right) x_{1}^{d-k} x_{2}^{k}$. To find $\|\mathcal{S}\|_{\sigma, \mathbb{F}}$ we need to maximize $|A|\left(\begin{array}{l}d \\ k\end{array}\right)\left|x_{1}\right|^{d-k}\left|x_{2}\right|^{k}$ subject to $\left|x_{1}\right|^{2}+\left|x_{2}\right|^{2}=1$. The maximum is obtained for $\left|x_{1}\right|^{2}=1-\frac{k}{d},\left|x_{2}\right|^{2}=\frac{k}{d}$. This proves (28).

Second assume that $\phi(z)$ has two distinct zeros: $\phi(z)=A(z+a)^{p}(z+b)^{q}$, where $a \neq b, p, q \in \mathbb{N}$ and $p+q \leqslant d$. Then

$$
r(z)=\frac{(z+a)^{p-1}(z+b)^{q-1}((p+q) z+p b+q a)}{(z+a)^{p-1}(z+b)^{q-1}(d(z+a)(z+b)-z((p+q) z+p b+q a))} .
$$

Suppose first that $p+q<d$. In order that $r(z)$ will be a Möbius transformation we need to assume that $(p+q) z+p b+q a$ divides $(z+a)(z+b)$. This is impossible, since $\phi^{\prime}$ has exactly $p+q-2$ common roots with $\phi$. Hence we are left with the case $p+q=d$. In this case

$$
r(z)=\frac{d z+\alpha}{\beta z+d a b}, \quad \alpha=p b+q a, \beta=d(a+b)-\alpha, p+q=d .
$$

The assumption that $g(z) \equiv z$ is equivalent to the following matrix equality

$$
\bar{B} B=\gamma^{2} I_{2}, \quad B=\left[\begin{array}{cc}
d & \alpha \\
\beta & d a b
\end{array}\right] \quad \gamma \neq 0 .
$$

Taking the determinant of the above identity we deduce that $\gamma^{4}=|\operatorname{det} B|^{2}>0$. So $\gamma^{2}= \pm \tau^{-2}$ for some $\tau>0$. Let $C=\tau B$. Suppose first that $\gamma^{2}=\tau^{-2}$. Then $\bar{C}$ is the inverse of $C$. So $\operatorname{det} C=\delta,|\delta|=1$. Then

$$
d a b=\delta d, d=\delta d \bar{a} \bar{b},-\alpha=\delta \bar{\alpha},-\beta=\delta \bar{\beta} .
$$

Hence $a b=\delta$.

We next observe that if we replace $\phi(z)$ with $\phi_{\theta}(z):=\phi\left(e^{\theta \mathbf{i}} z\right)$ for any $\theta \in \mathbb{R}$ we will obtain the following relations

Let

$$
p_{\theta}(z)=e^{\theta \mathbf{i}} p\left(e^{\theta \mathbf{i}} z\right), q_{\theta}(z)=q\left(e^{\theta \mathbf{i}} z\right), \overline{p_{\theta}}(z)=e^{-\theta \mathbf{i}} p\left(e^{-\theta \dot{\mathbf{i}}} z\right), \overline{q_{\theta}}(z)=\bar{q}\left(e^{-\theta \mathbf{i}} z\right) .
$$

$$
r_{\theta}(z)=\frac{p_{\theta}(z)}{q_{\theta}(z)}, \overline{r_{\theta}}(z)=\frac{\overline{p_{\theta}}(z)}{\overline{q_{\theta}}(z)}, g_{\theta}(z)=\overline{r_{\theta}}\left(r_{\theta}(z)\right) .
$$

A straightforward calculation shows that $g_{\theta}(z)=z$ for all $\theta \in \mathbb{R}$. Note the two roots of $\phi_{\theta}(z)$ are $-a e^{-\theta \mathbf{i}},-b e^{-\theta \mathbf{i}}$. Hence we can choose $\theta$ such that $\delta=-1$. Assume for simplicity of the exposition this condition holds for $\theta=0$, i.e., for $\phi$. So $\alpha$ and $\beta$ are real. In particular $a+b$ is real. So $b=-a^{-1}$ and $a-a^{-1}$ is real. Hence $a$ is real and also $b$ is real.

Suppose now that $\gamma^{2}=-\tau^{2}$. Then $\bar{B}=-B^{-1}$. So $\operatorname{det} B=\delta,|\delta|=1$. Then

$$
d a b=-\delta d, d=-\delta d \bar{a} \bar{b}, \alpha=\delta \bar{\alpha}, \beta=\delta \bar{\beta} .
$$

By considering $\phi_{\theta}$ as above we may assume that $\delta=1$ which gives again that $a \in \mathbb{R} \backslash\{0\}$ and $b=-a^{-1}$. This proves (29).

Vice versa, assume that $\phi(z)$ is of the form (29), where $a \in \mathbb{R} \backslash\{0\}$ and $b=-\frac{1}{a}$. We claim that $\bar{r}(r(z)) \equiv z$. The above arguments show that $r(z)=(d z+\alpha) /(\beta z+d a b)$. Furthermore

$$
a b=-1, \alpha=(d-p) a-\frac{p}{a}, \beta=p a-\frac{d-p}{a}, p \in[d-1] .
$$

Consider the matrix $B$ as given above. Note that the trace of this matrix is zero. We claim that $B$ is not singular. Indeed

$$
\begin{aligned}
& \operatorname{det} B=-\left(d^{2}+\alpha \beta\right)=-\left(d^{2}-(d-p)^{2}-p^{2}+p(d-p)\left(a^{2}+a^{-2}\right)\right)= \\
& -p(d-p)\left(a+a^{-1}\right)^{2} .
\end{aligned}
$$


Hence $B$ has two distinct eigenvalues $\{\gamma,-\gamma\}$ and is diagonalizable. As $B$ is a real matrix we get that $\bar{B} B=B^{2}=\gamma^{2} I_{2}$. Therefore $\bar{r}(r(z)) \equiv z$. As $\bar{r}_{\theta}\left(r_{\theta}(z)\right) \equiv z$ for each $s \in \mathbb{R}$ we deduce that for each $\phi$ of the form (29) $z q(z)-p(z)$ is a zero polynomial.

7.2. Computation of $\|\mathcal{S}\|_{\sigma}$ in the exceptional cases. Assume now that $\mathrm{S}_{\theta} \in \mathrm{S}^{d} \mathbb{C}^{2}$ is induced by $\phi$ of the form (29). Then

$$
\mathcal{S}_{\theta} \times \otimes^{d} \mathbf{x}=A\left(x_{2}+c e^{-\theta \mathbf{i}} x_{1}\right)^{p}\left(x_{2}-c^{-1} e^{-\theta \mathbf{i}} x_{1}\right)^{d-p}, c \in \mathbb{R} \backslash\{0\}, \theta \in \mathbb{R}, p \in[d-1],
$$

Clearly, $\left\|\mathcal{S}_{\theta}\right\|_{\sigma}=\left\|\mathcal{S}_{0}\right\|_{\sigma}$. Thus it is enough to find $\left\|\mathcal{S}_{0}\right\|_{\sigma}$. For simplicity of notation we let $\mathcal{S}=\mathcal{S}_{0}$.

We now suggest the following simple approximations to find $\|\mathcal{S}\|_{\sigma}$ using the case (2) of Theorem 18 It is enough to assume that $A=1$, i.e., $\mathcal{S}$ corresponds to $\phi(z)=(z+c)^{p}\left(z-c^{-1}\right)^{d-p}$. Let $\omega$ be a rational in the interval $(0,1)$. Set $\phi(z, \omega)=\phi(z)+\omega$. Let $\mathcal{S}(\omega) \in \mathrm{S}^{d} \mathbb{R}^{2}$ be the symmetric tensor corresponding to $\phi(z, \omega)$. Then $S(\omega) \times\left(\otimes^{d} \mathbf{x}\right)=\left(x_{2}+c x_{1}\right)^{p}\left(x_{2}-c^{-1} x_{1}\right)^{p-d}+\omega x_{1}^{d}$. By choosing $\mathbf{x}=(0,1)^{\top}$ we deduce that $\|\mathcal{S}(\omega)\|_{\sigma} \geqslant 1$. It is straightforward to show that $\|\mathcal{S}(\omega)-\mathcal{S}\|_{\sigma}=\omega$. As $\omega \leqslant \omega\|\mathcal{S}(\tau)\|_{\sigma}$ we obtain

$$
\|\mathcal{S}\|_{\sigma} \in\left[\|\mathcal{S}(\omega)\|_{\sigma}(1-\omega),\|\mathcal{S}(\omega)\|_{\sigma}(1+\omega)\right]
$$

Observe next that $\phi^{\prime}(z, \omega)=\phi^{\prime}(z)$. Hence a common root of $\phi(z, \omega)$ and $\phi^{\prime}(z, \omega)$ can not be a common root of $\phi(z)$ and $\phi^{\prime}(z)$. Therefore $\phi(z, \omega)$ and $\phi^{\prime}(z, \omega)$ can have at most one common root. As $d \geqslant 3$ we deduce that $\mathcal{S}(\omega)$ does not satisfy the assumptions of part (2) of Theorem 18 .

Assume that $c \in \mathbb{Q}$. Let $\delta$ be rational in the interval $(0,1)$ and assume that $\omega=\delta / 4$. Use the case (1) of Theorem 19 to find an approximation $L(\mathcal{S}(\omega))$ that satisfies $\left|\|\mathcal{S}(\omega)\|_{\sigma}-L(\mathcal{S}(\omega))\right| \leqslant(\delta / 4)\|\mathcal{S}(\omega)\|$. Let us take $L(\mathcal{S}(\omega))$ to be an approximation for $\|\mathcal{S}\|$. Then

$$
\left|\|\mathcal{S}\|_{\sigma}-L(\mathcal{S}(\omega))\right| \leqslant\left|\|\mathcal{S}\|_{\sigma}-\|\mathcal{S}(\omega)\|_{\sigma}\right|+\left|\|\mathcal{S}(\omega)\|_{\sigma}-L(\mathcal{S}(\omega))\right| \leqslant(\omega+\delta / 4)\|\mathcal{S}(\omega)\|_{\sigma} \leqslant \frac{\delta}{2} \frac{1}{1-\delta / 4}\|\mathcal{S}\|_{\sigma}<\delta\|\mathcal{S}\|_{\sigma} .
$$

Thus we found a rational approximation of $\|\mathcal{S}\|_{\sigma}$ that satisfies the inequality of Theorem 19 for $\mathbb{F}=\mathbb{C}$. The complexity of $L(\mathcal{S}(\omega))$ is given in case (1) of Theorem 19 .

\section{NumERICAL EXAMPLES}

In this section we give some numerical examples of applications of Theorem 18 for the qubit cases, and of Theorem 7 for $n>2$. In many examples here $f(\mathbf{x})$ is a sum of two monomials. In this case we show that to compute the complex spectral norm of $f$ one can assume that the coefficients of the two monomials are nonnegative. Equivalently, we can assume the symmetric tensor $\mathcal{S}$ has nonnegative entries. Hence $\|\mathcal{S}\|_{\sigma}=\|\mathcal{S}\|_{\sigma, \mathbb{R}}=\|f\|_{\sigma}\left[26\right.$. However, if $f$ has real coefficients then it may happen that $\|f\|_{\sigma, \mathbb{R}}<\|f\|_{\sigma}$. See Example 2 below.

Lemma 20. Let $f \in \mathrm{P}(d, n, \mathbb{C})$, where $d, n \geqslant 2$, and assume that $f=a \mathbf{x}^{\mathbf{j}}+b \mathbf{x}^{\mathbf{k}}$, where $\mathbf{j} \neq \mathbf{k}$. Denote $g(\mathbf{x})=|a| \mathbf{x}^{\mathbf{j}}+|b| \mathbf{x}^{\mathbf{k}}$. Then $\|f\|_{\sigma}=\|g\|_{\sigma}=\|g\|_{\sigma, \mathbb{R}}=g(\mathbf{y})$ for some $\mathbf{y} \geqslant \mathbf{0},\|\mathbf{y}\|=1$.

Proof. Clearly, it is enough to assume that $a \neq 0$ and $b \neq 0$. Let $\mathbf{x}=\left(x_{1}, \ldots, x_{n}\right)^{\top}$ and denote $\mathbf{x}_{+}=$ $\left(\left|x_{1}\right|, \ldots,\left|x_{n}\right|\right)^{\top}$. Note that $\|\mathbf{x}\|=\left\|\mathbf{x}_{+}\right\|$. Assume that $\|\mathbf{x}\|=1$. As $|f(\mathbf{x})|,|g(\mathbf{x})| \leqslant g\left(\mathbf{x}_{+}\right)$it follows that $\|f\|_{\sigma} \leqslant\|g\|_{\sigma}=g(\mathbf{y})$, for some $\mathbf{y} \geqslant 0$ and $\|\mathbf{y}\|=1$. Hence $\|g\|_{\sigma}=\|g\|_{\sigma, \mathbb{R}}$. Observe next that $f(\mathbf{x})=\mathbf{x}^{\mathbf{l}}\left(a \mathbf{x}^{\mathbf{j}^{\prime}}+b \mathbf{x}^{\mathbf{k}^{\prime}}\right)$. We can choose $\mathbf{l} \geqslant 0$ so that the monomials $\mathbf{x}^{\mathbf{j}^{\prime}}$ and $\mathbf{x}^{\mathbf{k}^{\prime}}$ do not have a common variable. Assume that $\mathbf{z}=\left(z_{1}, \ldots, z_{n}\right)^{\top}$ such that $\mathbf{z}_{+}=\mathbf{y}$. Then it is possible to choose the arguments of $z_{1}, \ldots, z_{n}$ such that $\left|a \mathbf{z}^{\mathbf{j}^{\prime}}+b \mathbf{z}^{\mathbf{k}^{\prime}}\right|=|a| \mathbf{y}^{\mathbf{j}^{\prime}}+|b| \mathbf{y}^{\mathbf{k}^{\prime}}$. Hence $|f(\mathbf{z})|=g(\mathbf{y})=\|g\|_{\sigma}$. Therefore $\|f\|_{\sigma}=\|g\|_{\sigma}$.

In some of the examples we discuss, we modified the examples of $f$, that satisfy conditions of Lemma 20 , by considering $f_{e}$ :

$$
\begin{aligned}
& f_{e}(\mathbf{x})=\sqrt{1-|e|^{2}} f(\mathbf{x})+e h(\mathbf{x}), \quad e=t \omega, \\
& t=0,1 / 5,1 / 4 /, 1 / 3,1 / 2,1, \quad \omega=1,-1, i, 1 / 2+i \frac{\sqrt{3}}{2},-1 / 2+i \frac{\sqrt{3}}{2}, \\
& f=a \mathbf{x}^{\mathbf{j}}+b \mathbf{x}^{\mathbf{k}}, h=c \mathbf{x}^{\mathbf{1}}, \mathbf{j} \neq \mathbf{k}, \mathbf{j} \neq \mathbf{l}, \mathbf{k} \neq \mathbf{l}, a, b, c>0,\|f\|=\|h\|=1 .
\end{aligned}
$$

Note that $\left\|f_{e}\right\|=1$, and $f_{0}=f, f_{1}=h$. Hence, when we give our results for $\left\|f_{e}\right\|_{\sigma}$ we give separately the values of $\|f\|_{\sigma},\|h\|_{\sigma}$, and $\left\|f_{e}\right\|_{\sigma}$ for $t=1 / 5,1 / 4 /, 1 / 3,1 / 2$ and the above values of $\omega$. 
First we discuss the symmetric $d$-qubits. If the $d$-qubits are real we compute both the complex and real spectral norms of a given tensor $\mathcal{S}$. In Theorem 18, we showed that the spectral norm of a given $d$-qubit can be found by solving the polynomial equation $z v(z)-u(z)=0$ (see (27)), which is of degree of at most $(d-1)^{2}+1$. In what follows that we assume that the polynomial $z v(z)-u(z)$ is not a zero polynomial. (That is, we are not dealing with the exceptional cases that are discussed in 87) We use formula (30) to find $\|\mathcal{S}\|_{\sigma}$, and formula (31) to find $\|\mathcal{S}\|_{\sigma, \mathbb{R}}$.

For $n>2$ we use Theorem 7 to compute $\|f\|_{\sigma}$. Thus we need to assume that fix $(\mathbf{H})$ is finite, or if all the monomials of $f$ have nonnegative coefficents then the set of the real fixed points of $\mathbf{F}$ is finite.

In this paper we use Bertini [6] (version 1.5, released in 2015), which is a well developed software to find all the complex solutions of a given polynomail system. It is worth noting that, in contrast to the theoretical results of this paper, the output of Bertini is not certified and may be incorrect by an unbounded error if the software jumps between solutions when tracking the homotopy paths.

All the computation are implemented with Matlab R2018b on a MacBook Pro 64-bit OS X (10.12.6) system with $16 \mathrm{~GB}$ memory and $2.9 \mathrm{GHz}$ Intel Core i7 CPU. In the display of the computational results, only four decimal digits are shown. The default parameters in Bertini are used to solve the polynomial equation $z v(z)-u(z)=0$. Since all examples only take few seconds we will not show the computing time.

In our examples all polynomials correspond to the symmetric tensors $\mathcal{S} \in \mathrm{S}^{d} \mathbb{C}^{n}$ of Hilbert-Schmidt norm one. Furthermore, if $\mathcal{S}$ has nonnegative entries then $\|\mathcal{S}\|_{\sigma}=\|\mathcal{S}\|_{\sigma, \mathbb{R}}[26$.

Assume that $d=2$. Then $\left|s_{d}\right|=\left|\mathcal{S}_{2, \ldots, 2}\right|$, and if $\mathcal{S}$ has nonnegative entries then one can use part (2) of Theorem 18. In these examples we find the degree of the polynomial $z v(z)-u(z)$, the number of real and complex roots, and the number of roots that fail to satisfy (24).

8.1. Three examples of symmetric 3-qubits. These examples are interesting to us, and some of them are discussed in other papers.

Example 1. [48] Let $f=0.3104 x_{1}^{3}-1.4598 x_{1}^{2} x_{2}-0.6558 x_{1} x_{2}^{2}+0.2235 x_{2}^{3}$. The polynomial $z v(z)-u(z)$ has degree $5=2^{2}+1$. It has 5 roots, 3 of them are real and the other 2 are complex. We have $R=R_{1}, R^{\prime}=R_{1}^{\prime}$. Then

$$
\|f\|_{\sigma} \approx 0.7027, \quad\|f\|_{\sigma, \mathbb{R}} \approx 0.6205 .
$$

Example 2. 26] Let $f=\frac{3}{2} x_{1}^{2} x_{2}-\frac{1}{2} x_{2}^{3}$. The polynomial $z v(z)-u(z)$ has degree 4 . It has 4 roots, 2 of them are real and the other 2 are complex. We have $R=R_{1}, R^{\prime}=R_{1}^{\prime}$. Then

$$
\|f\|_{\sigma} \approx 0.7071, \quad\|f\|_{\sigma, \mathbb{R}}=0.5 \text {. }
$$

Example 3. Let $f=\frac{1}{\sqrt{5}} x_{1}^{3}-\frac{3}{2 \sqrt{5}} x_{1}^{2} x_{3}-\frac{3}{\sqrt{5}} x_{1} x_{2}^{3}+\frac{1}{2 \sqrt{5}} x_{2}^{3}$. The polynomial $z v(z)-u(z)$ has degree 5 . It has 5 roots, 3 of them are real and the other 2 are complex. We have $R=R_{1}, R^{\prime}=R_{1}^{\prime}$. Then

$$
\|f\|_{\sigma} \approx 0.7071, \quad\|f\|_{\sigma, \mathbb{R}} \approx 0.5000 \text {. }
$$

8.2. Five examples from [3] and their variations. In [3] the authors give examples of $d$-symmetric qubits for $d=4, \ldots, 12$, which they assume to have the lowest complex spectral norm. Their examples are motivated by the Majorana model, see Appendix 3. (Note that some examples have at least two versions (a) and (b).) Our software gave the same values of the spectral norms for the examples in [3]. We could not find with our software examples of symmetric $d$-qubits with lower complex spectral norm. In the following examples we find the spectral norm of $f_{e}$ of the form (33), where $f$ is the polynomial given in [3]. The polynomial $h$ corresponds to a monomial given by Dicke basis with the lowest spectral norm. (See Appendix 1.)

Example 4. [3, Corresponds to example 6.1] Let $f=\frac{1}{\sqrt{3}} x_{1}^{4}+\frac{\sqrt{8}}{\sqrt{3}} x_{1} x_{2}^{3}$. The polynomial $z v(z)-u(z)$ has degree $10=3^{2}+1$. It has 10 roots, 4 of them are real and the other 6 are complex. We have $R=R_{1}$, $R^{\prime}=R_{1}^{\prime}$. Then $\|f\|_{\sigma} \approx 0.5774$. According to [3], $\|f\|_{\sigma}=\frac{1}{\sqrt{3}}$. Let $h=\sqrt{6} x_{1}^{2} x_{2}^{2}$. Then $\|h\|_{\sigma}=\frac{\sqrt{3}}{\sqrt{8}} \approx 0.6124$. (See (35).) Table 1 gives the results for $\left\|f_{e}\right\|_{\sigma}$ :

Example 5. 3. Corresponds to example 6.2(b), figure 5(b)] Let $f=\frac{1}{\sqrt{1+A^{2}}} x_{1}^{5}+\frac{\sqrt{5} A}{\sqrt{\left(1+A^{2}\right)}} x_{1} x_{2}^{4}$, where $A \approx$ 1.53154. The polynomial $z v(z)-u(z)$ has degree $17=4^{2}+1$. It has 17 roots, 5 of them are real and the other 


\begin{tabular}{|c|c|c|c|c|}
\hline$\left\|f_{e}\right\|_{\sigma}$ & $t=\frac{1}{5}$ & $t=\frac{1}{4}$ & $t=\frac{1}{3}$ & $t=\frac{1}{2}$ \\
\hline$\omega=1$ & 0.6787 & 0.7012 & 0.7358 & 0.7918 \\
\hline$\omega=-1$ & 0.6314 & 0.6442 & 0.6645 & 0.6989 \\
\hline$\omega=i$ & 0.6662 & 0.6863 & 0.7172 & 0.7676 \\
\hline$\omega=\frac{1}{2}+\frac{\sqrt{3}}{2} i$ & 0.6314 & 0.6442 & 0.6645 & 0.6989 \\
\hline$\omega=-\frac{1}{2}+\frac{\sqrt{3}}{2} i$ & 0.6787 & 0.7012 & 0.7358 & 0.7918 \\
\hline
\end{tabular}

TABle 1. Computational Results of $\left\|f_{e}\right\|_{\sigma}$ for Example 4 with different $t$ and $\omega$.

12 are complex. Four roots do not satisfy (24). Furthermore $R^{\prime}=R_{1}^{\prime}$. Then $\|f\|_{\sigma}=0.5467$. According to [3], $\|f\|_{\sigma}=\frac{1}{\sqrt{1+A^{2}}}$. Let $h=\sqrt{10} x_{1}^{3} x_{2}^{2}$. Then $\|h\|_{\sigma}=\frac{6 \sqrt{6}}{25} \approx 0.5879$. (See (35).) Here is the table for $\left\|f_{e}\right\|_{\sigma}$ :

\begin{tabular}{|c|c|c|c|c|}
\hline$\left\|f_{e}\right\|_{\sigma}$ & $t=\frac{1}{5}$ & $t=\frac{1}{4}$ & $t=\frac{1}{3}$ & $t=\frac{1}{2}$ \\
\hline$\omega=1$ & 0.5930 & 0.6038 & 0.6214 & 0.6573 \\
\hline$\omega=-1$ & 0.5930 & 0.6038 & 0.6214 & 0.6573 \\
\hline$\omega=i$ & 0.5622 & 0.5692 & 0.5793 & 0.5941 \\
\hline$\omega=\frac{1}{2}+\frac{\sqrt{3}}{2} i$ & 0.5759 & 0.5830 & 0.5941 & 0.6133 \\
\hline$\omega=-\frac{1}{2}+\frac{\sqrt{3}}{2} i$ & 0.5759 & 0.5830 & 0.5941 & 0.6133 \\
\hline
\end{tabular}

TABLE 2. Computational Results of $\left\|f_{e}\right\|_{\sigma}$ for Example 5 with different $t$ and $\omega$.

Example 6. 3, Corresponds to example 6.3] Let $f=\sqrt{3}\left(x_{1}^{5} x_{2}+x_{1} x_{2}^{5}\right)$. The polynomial $z v(z)-u(z)$ has degree $25<5^{2}+1$. It has 25 roots, 7 of them are real and the other 18 are complex. For the 25 roots $z$, (24) fails to hold for 5 roots. Six of the seven real roots satisfy $z q(z)-p(z)=0$. Then $\|f\|_{\sigma}=0.4714$. According to [3], $\|f\|_{\sigma}=\frac{\sqrt{2}}{3}$. Let $h=\sqrt{20} x_{1}^{3} x_{2}^{3}$. Then $\|h\|_{\sigma}=\frac{\sqrt{5}}{4} \approx 0.5590$. (See (35).) Here is the table for $\left\|f_{e}\right\|_{\sigma}$ :

\begin{tabular}{|c|c|c|c|c|}
\hline$\left\|f_{e}\right\|_{\sigma}$ & $t=\frac{1}{5}$ & $t=\frac{1}{4}$ & $t=\frac{1}{3}$ & $t=\frac{1}{2}$ \\
\hline$\omega=1$ & 0.5382 & 0.5590 & 0.5946 & 0.6545 \\
\hline$\omega=-1$ & 0.5382 & 0.5590 & 0.5946 & 0.6545 \\
\hline$\omega=i$ & 0.4777 & 0.4811 & 0.4886 & 0.5076 \\
\hline$\omega=\frac{1}{2}+\frac{\sqrt{3}}{2} i$ & 0.5054 & 0.5148 & 0.5312 & 0.5688 \\
\hline$\omega=-\frac{1}{2}+\frac{\sqrt{3}}{2} i$ & 0.5054 & 0.5148 & 0.5312 & 0.5688 \\
\hline
\end{tabular}

TABle 3. Computational Results of $\left\|f_{e}\right\|_{\sigma}$ for Example [6] with different $t$ and $\omega$.

Example 7. [3, Corresponds to example 6.4] Let $f=\frac{\sqrt{7}}{\sqrt{2}}\left(x_{1}^{6} x_{2}+x_{1} x_{2}^{6}\right)$. The polynomial $z v(z)-u(z)$ has degree $36<6^{2}+1$. It has 36 roots, 6 of them are real and the other 30 are complex. For the 36 roots $z$, (24) fails to hold for 11 roots. Five of the six real roots satisfy $z q(z)-p(z)=0$. Then $\|f\|_{\sigma}=0.4508$. Let $h=\sqrt{35} x_{1}^{4} x_{2}^{3}$. Then $\|h\|_{\sigma}=\frac{48 \sqrt{15}}{7^{3}} \approx 0.5420$. (See (35).) Here is the table for $\left\|f_{e}\right\|_{\sigma}$ :

\begin{tabular}{|c|c|c|c|c|}
\hline$\left\|f_{e}\right\|_{\sigma}$ & $t=\frac{1}{5}$ & $t=\frac{1}{4}$ & $t=\frac{1}{3}$ & $t=\frac{1}{2}$ \\
\hline$\omega=1$ & 0.5006 & 0.5131 & 0.5346 & 0.5796 \\
\hline$\omega=-1$ & 0.4939 & 0.5048 & 0.5229 & 0.5597 \\
\hline$\omega=i$ & 0.4988 & 0.5109 & 0.5314 & 0.5742 \\
\hline$\omega=\frac{1}{2}+\frac{\sqrt{3}}{2} i$ & 0.4998 & 0.5121 & 0.5332 & 0.5772 \\
\hline$\omega=-\frac{1}{2}+\frac{\sqrt{3}}{2} i$ & 0.4975 & 0.5092 & 0.5291 & 0.5703 \\
\hline
\end{tabular}

TABLE 4. Computational Results of $\left\|f_{e}\right\|_{\sigma}$ for Example 7 with different $t$ and $\omega$.

Example 8. [3, Corresponds to example 6.5] Let $f=4\left(0.336 \sqrt{2} x_{1}^{7} x_{2}+0.3705 \sqrt{7} x_{1}^{2} x_{2}^{6}\right)$. The polynomial $z v(z)-u(z)$ has degree $42<7^{2}+1$, which has 41 roots, 7 of them are real and the other 34 are complex. One of the real roots $z=0$ has multiplicity 2. For the $41 \operatorname{roots} z$, (24) fails to hold for 10 roots. Then $\|f\|_{\sigma}=0.4288$. Let $h=\sqrt{70} x_{1}^{4} x_{2}^{4}$. Then $\|h\|_{\sigma}=\frac{\sqrt{70}}{16} \approx 0.5229$. (See (35).) Here is the table for $\left\|f_{e}\right\|_{\sigma}$ :

More details on the above examples and additional examples for $n=2$ are given in [30]. 


\begin{tabular}{|c|c|c|c|c|}
\hline$\left\|f_{e}\right\|_{\sigma}$ & $t=\frac{1}{5}$ & $t=\frac{1}{4}$ & $t=\frac{1}{3}$ & $t=\frac{1}{2}$ \\
\hline$\omega=1$ & 0.4946 & 0.5108 & 0.5374 & 0.5867 \\
\hline$\omega=-1$ & 0.4841 & 0.4979 & 0.5206 & 0.5630 \\
\hline$\omega=i$ & 0.4919 & 0.5075 & 0.5330 & 0.5806 \\
\hline$\omega=\frac{1}{2}+\frac{\sqrt{3}}{2} i$ & 0.4934 & 0.5093 & 0.5354 & 0.5840 \\
\hline$\omega=-\frac{1}{2}+\frac{\sqrt{3}}{2} i$ & 0.4898 & 0.5049 & 0.5297 & 0.5759 \\
\hline
\end{tabular}

TABle 5. Computational Results of $\left\|f_{e}\right\|_{\sigma}$ for Example 8 with different $t$ and $\omega$.

8.3. A family of symmetric 3-qutrits. Let us consider the following family of symmetric 3-qutrits.

$$
f_{a, b}\left(x_{1}, x_{2}, x_{3}\right)=a\left(x_{1}^{3}+x_{2}^{3}+x_{3}^{3}\right)+b x_{1} x_{2} x_{3}, \quad 3|a|^{2}+|b|^{2} / 6=1 .
$$

This example is inspired by [13, Example (1)]. It is straightforward to show that

$$
\left\|f_{1 / \sqrt{3}, 0}\right\|_{\sigma}=\left\|f_{1 / \sqrt{3}, 0}\right\|_{\sigma, \mathbb{R}}=\frac{1}{\sqrt{3}} \approx 0.5774, \quad\left\|f_{0, \sqrt{6}}\right\|_{\sigma}=\left\|f_{0, \sqrt{6}}\right\|_{\sigma, \mathbb{R}}=\frac{\sqrt{2}}{3}=0.4714 .
$$

Note that $f_{0, \sqrt{6}}$ corresponds to the most entangled Dicke basis $\mathcal{S}(3,3)$ (34). Observe that $\left\|f_{a, b}\right\|_{\sigma}=\left\|f_{\bar{a}, \bar{b}}\right\|_{\sigma}=$ $\left\|f_{\zeta a, \zeta b}\right\|_{\sigma}$ for $|\zeta|=1$. Here is the table for $\left\|f_{a, b}\right\|_{\sigma}$ :

\begin{tabular}{|c|c|c|c|c|c|c|}
\hline$a$ & $b$ & No. of real fixed points & No. of complex fixed points & $\left\|f_{a, b}\right\|_{\sigma, \mathbb{R}}$ & $\left\|f_{a, b}\right\|_{\sigma}$ \\
\hline$\frac{1}{3}$ & 2 & 8 & 56 & 0.5774 & 0.5774 \\
\hline$\frac{1}{2}$ & $\sqrt{\frac{3}{2}}$ & 8 & 56 & 0.5244 & 0.5244 \\
\hline$\frac{1}{3}$ & -2 & 8 & 56 & 0.4975 & 0.5092 \\
\hline$\frac{1}{2}$ & $-\sqrt{\frac{3}{2}}$ & 8 & 56 & 0.5000 & 0.5000 \\
\hline 0 & $\sqrt{6}$ & 5 & 50 & 0.4714 & 0.4714 \\
\hline$\frac{1}{\sqrt{3}}$ & 0 & 8 & 56 & 0.5774 & 0.5774 \\
\hline$\frac{1}{6}+\frac{\sqrt{3}}{6} i$ & $\sqrt{2}-\sqrt{2} i$ & 1 & 63 & - & 0.5730 \\
\hline$\frac{1}{4}+\frac{\sqrt{3}}{4} i$ & $\frac{\sqrt{6}}{4}+\frac{3 \sqrt{2}}{4} i$ & 1 & 63 & - & 0.5244 \\
\hline
\end{tabular}

TABLE 6. Computational Results for 8.3 with different $a$ and $b$.

Let us consider the real case. For different real $a$ and $b$, we use Bertini to solve the equation $F(y)=y$, and get the real spectral norm of tensor $f_{a, b}$. Results are shown in the Table [6. As we see, the number of real fixed point is $8=(3-1)^{3}$ the maximum possible as given by part (3) of Theorem 7 , except for $f_{0, \sqrt{6}}$ corresponding to $\mathcal{S}(3,3)$. Thus, among all these examples the Dicke state $\mathcal{S}(3,3)$ is the most entangled one.

8.4. A family of symmetric 4-ququadrits. Recall that most entangled 3-qubit is $\mathcal{W} \in \mathrm{S}^{3} \mathbb{C}^{2}$, which corresponds to $f=\sqrt{3} x_{1}^{2} x_{2}$ [54, 12]. (It is the Dicke basis element $\mathcal{S}(3,2)$.) It is of interest to consider the tensor product state $\mathcal{W} \otimes \mathcal{W} \in \otimes{ }^{6} \mathbb{C}^{2}$ [10, 11. Note that this state is not symmetric. Lemma 2.3 in [16] yields

$$
\|\mathcal{W} \otimes \mathcal{W}\|_{\sigma}=\|\mathcal{W}\|_{\sigma}^{2}=\|\mathcal{S}(3,2)\|^{2}=\left(\frac{2}{3}\right)^{2}=\frac{4}{9} \approx 0.4444
$$

It is possible to represent $\mathcal{W} \otimes \mathcal{W}$ as a symmetric tensor $\mathcal{S} \in \mathrm{S}^{3} \mathbb{C}^{4}$. It is represented by polynomial $f=x_{1}^{2} x_{4}+2 x_{1} x_{2} x_{3}$ [10. Let us consider $f_{a, b}=a x_{1}^{2} x_{4}+2 b x_{1} x_{2} x_{3}$, where $|a|^{2}+2|b|^{2}=3$. (So $\left\|f_{a, b}\right\|=1$.) Note that $f=f_{1,1}$. In Table 7 , we show $\left\|f_{a, b}\right\|_{\sigma}$ for some values of $a, b$. These computations point

\begin{tabular}{|c|c|c|}
\hline$a$ & $b$ & $\left\|f_{a, b}\right\|_{\sigma}$ \\
\hline 1 & 1 & 0.4444 \\
\hline$\frac{\sqrt{2}}{2}$ & $\frac{\sqrt{5}}{2}$ & 0.4536 \\
\hline$\frac{\sqrt{5}}{2}$ & $\sqrt{\frac{7}{8}}$ & 0.4491 \\
\hline$\frac{\sqrt{2}}{2}+\frac{\sqrt{2}}{2} i$ & $\frac{1}{2}+\frac{\sqrt{3}}{2} i$ & 0.4444 \\
\hline$\frac{\sqrt{2}}{2}\left(\frac{1}{2}-\frac{\sqrt{3}}{2} i\right)$ & $\frac{\sqrt{5}}{2}\left(\frac{\sqrt{3}}{4}+\frac{\sqrt{13}}{4} i\right)$ & 0.4536 \\
\hline$\frac{\sqrt{6}}{2}\left(\frac{\sqrt{2}}{2}+\frac{\sqrt{2}}{2} i\right)$ & $\frac{\sqrt{3}}{2}\left(\frac{\sqrt{3}}{2}+\frac{1}{2} i\right)$ & 0.4714 \\
\hline
\end{tabular}

TABLE 7. Computational Results for Example 8.4 with different $a$ and $b$.

out that probably $\|\mathcal{S}\|=\|f\|_{\sigma}$ is equal to $\|\mathcal{W} \otimes \mathcal{W}\|_{\sigma}$. This contrast with the results in [10, 11 that $\operatorname{rank} \mathcal{S}=7<\operatorname{rank} \mathcal{W} \otimes \mathcal{W}=8<(\operatorname{rank} \mathcal{W})^{2}=9$. (Recall that the rank of a tensor $\mathcal{T}$ is the minimal number 
of summands in a representation of $\mathcal{T}$ as a sum of rank one tensors.) Next observe that $f$ does not have the minimal complex spectral norm in the above examples. Finally, $\|f\|_{\sigma}<\|\mathcal{S}(3,4)\|=\frac{\sqrt{2}}{3}$.

Acknowledgement: We thank the two referees for their useful remarks and comments.

\section{REFERENCES}

[1] S. Aaronson and A. Arkhipov. The computational complexity of linear optics, Theory Comput. 9 (2013), $143-252$.

[2] A. Auffinger, G. Ben Arous and J. Černý, Random matrices and complexity of spin glasses, Comm. Pure Appl. Math. 66 (2013), no. 2, 165-201.

[3] M. Aulbach, D. Markham and Mi. Murao, The maximally entangled symmetric state in terms of the geometric measure, New Journal of Physics, 12 (2010), 073025.

[4] S. Banach, "Über homogene Polynome in $\left(L^{2}\right)$," Studia Math., 7 (1938), pp. 36-44.

[5] M. Bardet, J.-C. Faugére and B. Salvy, On the complexity of the $F_{5}$ Gröbner basis algorithm, J. Symbolic Comput. 70 (2015), 49-70.

[6] Daniel J. Bates, Jonathan D. Hauenstein, Andrew J Sommese, and Charles W. Wampler. Bertini: Software for Numerical Algebraic Geometry. Available at bertini.nd.edu with permanent doi: dx.doi.org/10.7274/R0H41PB5.

[7] J.S. Bell, On the Einstein-Podolsky-Rosen paradox, Physics 1 (1964), 195-290.

[8] D. Cartwright, B. Sturmfels, The number of eigenvectors of a tensor, Linear Algebra Appl. 438 (2013), no. 2, 942-952.

[9] B. Chen, S. He, Z. Li, and S. Zhang, Maximum block improvement and polynomial optimization, SIAM J. OPTIM. $22(2012), 87-107$.

[10] L. Chen, E. Chitambar, R. Duan, Z. Ji, A. Winter, Phys. Rev. Lett. 105 (2010), 200501.

[11] L. Chen and S. Friedland, The tensor rank of tensor product of two three-qubit W states is eight, Linear Algebra and its Applications, 543 (2018), 1-16.

[12] L. Chen, A. Xu and H. Zhu, Computation of the geometric measure of entanglement for pure multiqubit states, Physical Review A 82 (2010), 032301.

[13] R. Cleve, D. Gottesman, and H.-K. Lo, How to share a quantum secret, Phys. Rev. Lett. 83 (1999), 648, arXiv:quant-ph/9901025 pdf

[14] M. Coste, Real Algebraic Sets, Lecture notes, 2005, http://perso.univ-rennes1.fr/michel.coste/polyens/RASroot.pdf

[15] L. de Lathauwer, B. de Moor, and J. Vandewalle, On the best rank-1 and rank- $\left(R_{1}, R_{2}, \ldots, R_{N}\right)$ approximation of higher-order tensors, SIAM J. Matrix Anal. Appl. 21 (2000), pp. 1324-1342.

[16] H. Derksen, S. Friedland, L.-H. Lim, and L. Wang, Theoretical and computational aspects of entanglement, arXiv:1705.07160

[17] H. Derksen and V. Makam, Highly entangled tensors, arXiv:1803.09788

[18] R. H. Dicke, Coherence in Spontaneous Radiation Processes, Physical Review. 93 (1) (1954), 99-110.

[19] J.-G. Dumas, C. Pernet and Z. Wan, Efficient computation of the characteristic polynomial, arXiv:cs/0501074.

[20] A. Einstein, B. Podolsky, and N. Rosen N, Can Quantum-Mechanical Description of Physical Reality Be Considered Complete?, Phys. Rev. 47, is. 10, (1935), 777-780.

[21] J.C. Faugére, P. Gianni, D. Lazard and T. Mora, Efficient computation of zero-dimensional Gröbner bases by change of ordering, J. Symbolic Comput. 16 (1993), no. 4, 329-344.

[22] W. Feller, An Introduction to Probability Theory and Its Applications, vol I, J. Wiley, 1958.

[23] S. Friedland, Inverse eigenvalue problems, Linear Algebra Appl. 17 (1977), 15-51.

[24] S. Friedland, Best rank-one approximation of real symmetric tensors can be chosen symmetric, Front. Math. China, 8 (2013), pp. 19-40.

[25] S. Friedland, Matrices: Algebra, Analysis and Applications, World Scientific, 596 pp., 2016, Singapore, http://www2.math.uic.edu/ friedlan/bookm.pdf

[26] S. Friedland and L.-H. Lim, Nuclear Norm of Higher Order Tensors, Mathematics of Computation, 87 (2018), 12551281.

[27] S. Friedland, V. Mehrmann, R. Pajarola and S.K. Suter, On best rank one approximation of tensors, Numer. Linear Algebra Appl. 20 (2013), 942-955.

[28] S. Friedland and G. Ottaviani, The number of singular vector tuples and uniqueness of best rank one approximation of tensors, Foundations of Computational Mathematics 14, 6 (2014), 1209-1242.

[29] S. Friedland and V. Tammali, Low-rank approximation of tensors, Numerical Algebra, Matrix Theory, DifferentialAlgebraic Equations and Control Theory, edited by P. Benner et all, Springer, 2015, 377-410, arXiv:1410.6089

[30] S. Friedland and L. Wang, Geometric measure of entanglement of symmetric d-qubits is polynomial-time computable, arXiv:1608.01354

[31] I.M. Gelfand, M.M. Kapranov and A.V. Zelevinsky, Discriminants, resultants, and multidimensional determinants, Birkhäuser, 1994.

[32] G.H. Golub and C.F. Van Loan, Matrix Computations, Johns Hopkins Studies in the Mathematical Sciences, 2013.

[33] D. Gross, S. T. Flammia, and J. Eisert, Most Quantum States Are Too Entangled To Be Useful As Computational Resources, Phys. Rev. Lett. 102, 190501, 2009.

[34] J. Heintz, Definability and fast quantifier elimination in algebraically closed fields, Theor. Comput. Sci. 24, 3 (1983), $239-277$. 
[35] A.Higuchi, A. Sudbery, How entangled can two couples get? Physics Letters A, 273(4) (2000), pp. 213-217.

[36] C. J. Hillar and L.-H. Lim, Most tensor problems are NP-hard, J. ACM, 60 (2013), no. 6, Art. 45, 39 pp.

[37] J, Hubbard and D. Schleicher, Multicorns are not path connected, in Frontiers in Complex Dynamics, p. 73-101,edited by A. Bonifant, M. Lyubich, S. Sutherland, Princeton University Press, 2014.

[38] R. Hübener, M. Kleinmann, T.-C. Wei, C. González-Guillén, and O. Gühne, Phys. Rev. A 80 (2009), 032324.

[39] E. Jung, M.-R. Hwang, H. Kim, M.-S. Kim, D. Park, J.-W. Son, and S. Tamaryan, Reduced state uniquely defines the Groverian measure of the original pure state, Phys. Rev. A 77, 062317 (2008).

[40] R. M. Karp, Reducibility among combinatorial problems, pp. 85-103, in R.E. Miller and J.W. Thatcher (Eds), Complexity of Computer Computations, Plenum, New York, NY, 1972.

[41] J. Kuczyński and H. Woźniakowski, Estimating the largest eigenvalue by the power and Lanczos algorithms with a random start, SIAM Journal on Matrix Analysis and Applications 13 (1992), no. 4, 1094-1122.

[42] A. Mantzaflaris, E. Schost and E. Tsigaridas, Sparse rational univariate representation, Proceedings of the 2017 ACM International Symposium on Symbolic and Algebraic Computation, 301-308, ACM, New York, 2017.

[43] J. Martin, O. Giraud, P.A. Braun, D. Braun and T. Bastin, Multiqubit symmetric states with high geometric entanglement Phys. Rev. A 81 (2010), 062347.

[44] T. Mora, Solving polynomial equation systems I, The Kronecker-Duval philosophy, Encyclopedia of Mathematics and its Applications 88, Cambridge University Press (2003).

[45] T. S. Motzkin and E. G. Straus, Maxima for graphs and a new proof of Túran, Canadian J. Math., 17 (1965), pp. 533540.

[46] C.A. Neff and J.H. Reif, An efficient algorithm for the complex roots problem, J. Complexity 12 (1996), no. 2, 81-115.

[47] A. Neville, C. Sparrow, R. Clifford, E. Johnston, P. M. Birchall, A. Montanaro, and A. Laing, Classical boson sampling algorithms with superior performance to near-term experiments, Nat. Phys. 13 (2017), 1153.

[48] J. Nie and L. Wang, Semidefinite relaxations for best rank-1 tensor approximations. SIAM Journal on Matrix Analysis and Applications, 35 (2014), no. 3, pp. 1155-1179.

[49] B. Reznick, Sums of even powers of real linear forms, Mem. Amer. Math. Soc., 96 (1992), Number 463.

[50] F. Rouillier, Solving zero-dimensional systems through the rational univariate representation, J. of Appl. Algebra in Engin., Comm. and Computing, 9(5):433-461, 1999.

[51] E. Schrödinger, Discussion of probability relations between separated systems, Mathematical Proceedings of the Cambridge Philosophical Society, 31 is. 4, (1935), 555-563.

[52] , E. Schrödinger, Probability relations between separated systems, Mathematical Proceedings of the Cambridge Philosophical Society, 32, is. 3, (1936), 446-452.

[53] J. J. Sylvester, On a remarkable discovery in the theory of canonical forms and of hyperdeterminants, Phil. Mag. 2 (1851), 391-410 ( Collected papers, Vol. I, Paper 41).

[54] S.Tamaryan, T.C. Wei and D. Park, Maximally entangled three-qubit states via geometric measure of entanglement, Physical Review A. 80(5) (2009), 052315.

[55] J.J. Thomson, On the Structure of the Atom: an Investigation of the Stability of the Periods of Oscillation of a number of Corpuscles arranged at equal intervals around the Circumference of a Circle with Application of the results to the Theory of Atomic Structure, Philosophical magazine, Series 6, Volume 7, Number 39, pp 237-265, March 1904.

[56] L.L. Whyte, Unique arrangements of points on a sphere, American Mathematical Monthly 59 (1952), 9, 606-611.

[57] T. Zhang and G. H. Golub, Rank-one approximation to high order tensors, SIAM J. Matrix Anal. Appl., 23 (2001), no. 2, pp. 534-550.

[58] B.L. van der Waerden, Modern algebra, vol. 2, 1950.

Appendix 1: Dicke states and their entanglement

Let $\mathcal{S}(\mathbf{j}), \mathbf{j} \in J(d, n)$ be the orthonormal basis in $\mathrm{S}^{d} \mathbb{F}^{n}$ given in the end of $\oint 3$. For $n=2$ this basis is called the Dicke basis [18]. Recall that $\mathcal{S}(\mathbf{j}) \times \otimes^{d} \mathbf{x}=\sqrt{c(\mathbf{j})} \mathbf{x}^{\mathbf{j}}$.

Let

$$
\begin{aligned}
& \mathcal{S}(d, n)=\mathcal{S}(\mathbf{j}), \mathbf{j}=\left(j_{1}, \ldots, j_{n}\right) \in J(d, n), \text { where } \\
& j_{1}=\cdots=j_{l}=\left\lfloor\frac{d}{n}\right\rfloor, j_{l+1}=\cdots=j_{n}=\left\lceil\frac{d}{n}\right\rceil, l=n\left\lceil\frac{d}{n}\right\rceil-d .
\end{aligned}
$$

In the following lemma we find the entanglement of each $\mathcal{S}(\mathbf{j}), \mathbf{j} \in J(d, n)$ and the maximum entanglement of these states.

Lemma 1. Assume that $n, d \geqslant 2$ are two positive integers. Then

(1) For each $\mathbf{j}=\left(j_{1}, \ldots, j_{n}\right) \in J(d, n)$ the following equality holds

$$
\eta(\mathcal{S}(\mathbf{j}))=\log _{2} d^{d}-\log _{2} d !+\sum_{k=1}^{n}\left(\log _{2} j_{k} !-\log _{2} j_{k}^{j_{k}}\right) .
$$


$(2)$

$$
\|\mathcal{S}(d, n)\|_{\sigma}=\sqrt{\frac{d !\left(\left\lfloor\frac{d}{n}\right\rfloor\right)^{l\left\lfloor\frac{d}{n}\right\rfloor}\left(\left\lceil\frac{d}{n}\right\rceil\right)^{(n-l)\left\lceil\frac{d}{n}\right\rceil}}{d^{d}\left(\left\lfloor\frac{d}{n}\right\rfloor !\right)^{l}\left(\left\lceil\frac{d}{n}\right\rceil !\right)^{n-l}}}, \quad \eta(\mathcal{S}(d, n))=\log _{2} \frac{d^{d}\left(\left\lfloor\frac{d}{n}\right\rfloor !\right)^{l}\left(\left\lceil\frac{d}{n}\right\rceil !\right)^{n-l}}{d !\left(\left\lfloor\frac{d}{n}\right\rfloor\right)^{l\left\lfloor\frac{d}{n}\right\rfloor}\left(\left\lceil\frac{d}{n}\right\rceil\right)^{(n-l)\left\lceil\frac{d}{n}\right\rceil}} .
$$

$(3)$

$$
\|\mathcal{S}(d, n)\|_{\sigma} \leqslant\|\mathcal{S}(\mathbf{j})\|_{\sigma}, \quad \eta(\mathcal{S}(\mathbf{j})) \leqslant \eta(\mathcal{S}(d, n)), \quad \text { for each } \mathbf{j} \in J(d, n) .
$$

(4) Assume that the integer $n \geqslant 2$ is fixed and $d \gg 1$. Then

$$
\eta(\mathcal{S}(d, n))=\frac{1}{2}\left((n-1) \log _{2} d-n \log _{2} n\right)+O\left(\frac{1}{d}\right) .
$$

Proof. (1) Clearly

$$
\left|\mathcal{S}\left(j_{1}, \ldots, j_{n}\right) \times \otimes^{d} \mathbf{x}\right|^{2}=\frac{d !}{j_{1} ! \cdots j_{n} !}\left(\left|x_{1}\right|^{2}\right)^{j_{1}} \cdots\left(\left|x_{n}\right|^{2}\right)^{j_{n}} .
$$

Use Lagrange multipliers to deduce that the maximum of the above function for $\|\mathbf{x}\|=1$ is achieved at the points $\left|x_{k}\right|^{2}=\frac{j_{k}}{j_{1}+\cdots+j_{n}}=\frac{j_{k}}{d}$ for $k \in[n]$. Banach's theorem (7) yields

$$
\left\|\mathcal{S}\left(j_{1}, \ldots, j_{n}\right)\right\|_{\sigma, \mathbb{R}}^{2}=\left\|\mathcal{S}\left(j_{1}, \ldots, j_{n}\right)\right\|_{\sigma}^{2}=\frac{d ! \prod_{k=1}^{n} j_{k}^{j_{k}}}{d^{d} \prod_{k=1}^{n} j_{k} !} .
$$

This establishes the expression for $\eta(\mathcal{S}(\mathbf{j}))$.

(2) Follows straightforward from the definition of $\mathcal{S}(d, n)$ in (34) and the proof of part (1).

(3) Let $a, b$ be nonnegative integers such that $a \leqslant b-2$. We claim that

$$
\frac{a ! b !}{a^{a} b^{b}}<\frac{(a+1) !(b-1) !}{(a+1)^{a+1}(b-1)^{b-1}} .
$$

Indeed, the above inequality is equivalent to

$$
\frac{a !(a+1)^{a+1}}{a^{a}(a+1) !}<\frac{(b-1) ! b^{b}}{(b-1)^{b-1} b !} \Longleftrightarrow\left(\frac{a+1}{a}\right)^{a}<\left(\frac{b}{b-1}\right)^{b-1} .
$$

As $0^{0}=1$ we deduce that the above inequalities hold for $a=0$ and $b \geqslant 2$. Assume that $a \geqslant 1$. Then the last inequality in the above displayed relation is equivalent to the well known statement that the sequence $\left(1+\frac{1}{m}\right)^{m}$ is a strictly increasing .

Consider $\left\|\mathcal{S}\left(j_{1}, \ldots, j_{n}\right)\right\|^{-2}$. Suppose that there exists $j_{p}, j_{q}$ such that $\left|j_{p}-j_{q}\right| \geqslant 2$. Without loss of generality we may assume that $j_{p} \leqslant j_{q}-2$. Let $j_{l}^{\prime}=j_{l}$ for $l \in[n] \backslash\{p, q\}$, and $j_{p}^{\prime}=j_{p}+1, j_{q}^{\prime}=j_{q}-1$. Then the above inequality yields that $\left\|\mathcal{S}\left(j_{1}, \ldots, j_{n}\right)\right\|^{-2}<\left\|\mathcal{S}\left(j_{1}^{\prime}, \ldots, j_{n}^{\prime}\right)\right\|^{-2}$. Hence the maximum value of $\left\|\mathcal{S}\left(j_{1}, \ldots, j_{n}\right)\right\|^{-2}$, where $\left\{j_{1}, \ldots, j_{n}\right\} \in J(d, n)$, is achieved for $\left\{j_{1}, \ldots, j_{n}\right\}$ satisfying $\left|j_{p}-j_{q}\right| \leqslant 1$ for all $p, q \in[n]$. Without loss of generality we can assume that $j_{1}, \ldots, j_{n}$ are given as in (34). This shows the inequality $\|\mathcal{S}(d, n)\|_{\sigma} \leqslant\|\mathcal{S}(\mathbf{j})\|_{\sigma}$. The definition (2) of $\eta(\mathcal{S})$ yields the inequality $\eta(\mathcal{S}(\mathbf{j})) \leqslant \eta(\mathcal{S}(d, n))$ for each $\mathbf{j} \in J(d, n)$.

(4). The expression for $\eta(\mathcal{S}(d, n))$ follows from Sterling's formula [22, p. 52] $k !=\sqrt{2 \pi k} k^{k} e^{-k} e^{\theta_{k} / 12 k}$, where $0<\theta_{k}<1$.

We now comment on the results given in Lemma 1. Parts (1)-(3) are well known for $n=2$ in physics community [3]. The states $\mathcal{S}\left(j_{1}, j_{2}\right)$ are called Dicke states. Note that

$$
\|\mathcal{S}(3,2)\|_{\sigma}=\frac{2}{3} \approx 0.6667,\|\mathcal{S}(4,2)\|_{\sigma}=\frac{\sqrt{6}}{4} \approx 0.61237,\|\mathcal{S}(5,2)\|_{\sigma}=\frac{6 \sqrt{6}}{25} \approx 0.5879 .
$$

It is known that the most entangled 3-qubit state with respect to geometric measure is $\mathcal{S}(3,2)$ [54, 12 . That is, the spectral norm of a nonsymmetric 3 -qubit is not less than the spectral norm of $\mathcal{S}(3,2)$, which is equivalent to the equality $\eta((2,2,2))=\eta(\mathcal{S}(3,2))$ as in (3). However, for $d>3$ the examples that are given in $₫ 8$ show that the states $\mathcal{S}(d, 2)$ are not the most entangled states in $\mathrm{S}^{d} \mathbb{C}^{2}$. See the Examples $i$ with , denoted as $\mathcal{E}_{i}$, in $₫ 8$ for $i=4-8$. 
It is shown in [16] that the most entangled 4-qubit is $\mathcal{M}_{4} \in \otimes^{4} \mathbb{C}^{2}$, which is not symmetric. (This was a conjecture of Higuchi-Sudbery [35].) Note that

$$
\left\|\mathcal{M}_{4}\right\|_{\sigma}=\frac{\sqrt{2}}{3} \approx 0.4714<\left\|\mathcal{E}_{5}\right\|_{\sigma} .
$$

According to [3], the symmetric state $\mathcal{E}_{5}$ is the most entangles 4-symmetric qubit.

Lemma 4.3.1 in [?] yields that

$$
\eta(\mathcal{S}) \leqslant \log _{2}\left(\begin{array}{c}
n+d-1 \\
n-1
\end{array}\right), \quad \mathcal{S} \in \mathrm{S}^{d} \mathbb{C}^{n},\|\mathcal{S}\|=1
$$

(See also 43.) In particular, for $n=2$ we have the inequality:

$$
\eta(\mathcal{S}) \leqslant \log _{2}(d+1), \quad \mathcal{S} \in \mathrm{S}^{d} \mathbb{C}^{2},\|\mathcal{S}\|=1 .
$$

Note that for a fixed $n$ and large $d$ we have the complexity expression

$$
\log _{2}\left(\begin{array}{c}
n+d-1 \\
n-1
\end{array}\right)=(n-1) \log _{2}(d+1)+\frac{(n-1)(n-2)}{2 \ln 2}-\log _{2}(n-1) !+O\left(\frac{1}{d}\right) .
$$

Let

$$
\eta_{\text {sym }}(d, n)=\max \left\{\eta(\mathcal{S}), \mathcal{S} \in \mathrm{S}^{d} \mathbb{C}^{n},\|\mathcal{S}\|=1\right\} .
$$

Combining the inequality (36) with part (3) of Lemma 1 we obtain

$$
\log _{2} \frac{d^{d}\left(\left\lfloor\frac{d}{n}\right\rfloor !\right)^{l}\left(\left\lceil\frac{d}{n}\right\rceil !\right)^{n-l}}{d !\left(\left\lfloor\frac{d}{n}\right\rfloor\right)^{l\left\lfloor\frac{d}{n}\right\rfloor}\left(\left\lceil\frac{d}{n}\right\rceil\right)^{(n-l)\left\lceil\frac{d}{n}\right\rceil}} \leqslant \eta_{\text {sym }}(d, n) \leqslant \log _{2}\left(\begin{array}{c}
n+d-1 \\
n-1
\end{array}\right), l=n\left\lceil\frac{d}{n}\right\rceil-d .
$$

In particular

$$
\log _{2} \frac{d^{d}\left(\left\lfloor\frac{d}{2}\right\rfloor !\right)\left(\left\lceil\frac{d}{2}\right\rceil !\right)}{d !\left(\left\lfloor\frac{d}{2}\right\rfloor\right)^{\left\lfloor\frac{d}{2}\right\rfloor}\left(\left\lceil\frac{d}{2}\right\rceil\right)^{\left\lceil\frac{d}{2}\right\rceil}} \leqslant \eta_{\text {sym }}(d, 2) \leqslant \log _{2}(d+1) .
$$

There is a gap of factor 2 between the lower and the upper bounds in (38) and (39) for fixed $n$ and $d \gg 1$. In [?] it is shown that the following inequality holds with respect to the corresponding Haar measure on the unit ball $\|\mathcal{S}\|=1$ in $\mathrm{S}^{d} \mathbb{C}^{2}$ :

$$
\operatorname{Pr}\left(\eta(\mathcal{S}) \leqslant \log _{2} d-\log _{2}\left(\log _{2} d\right)+\log _{2} 4-\log _{2} 5\right) \leqslant \frac{1}{d^{6}} .
$$

This shows that the upper bounds in (39) have the correct order. In particular, the above inequality is the analog of the inequality $\eta(\mathcal{T}) \geqslant d-2 \log _{2} d-2$ for most $d$-qubit states in [33.

Appendix 2: Computing isolated roots of certain polynomial systems

Let $g=g(\mathbf{x})$ be a nonconstant polynomial in $\mathbf{x}=\left(x_{1}, \ldots, x_{m}\right)^{\top} \in \mathbb{F}^{m}$. Denote by $d=\operatorname{deg} g$ the total degree of $g$. Then $g_{\pi} \in \mathrm{P}(d, m, \mathbb{F}) \backslash\{0\}$ denotes the homogeneous part of $d$ of degree $d$. That is, $g-g_{\pi}$ is a polynomial of degree less than $d$. In this Appendix we assume that $g_{i}(\mathbf{x})$ is a nonconstant polynomial of total degree $d_{i}=\operatorname{deg} g_{i, \pi} \in \mathbb{N}$ for $i \in[m]$. Our next assumption is that the following system of homogeneous equations has only the trivial solution

$$
g_{1, \pi}(\mathbf{x})=\cdots=g_{m, \pi}(\mathbf{x})=0, \mathbf{x} \in \mathbb{C}^{m} \Rightarrow \mathbf{x}=\mathbf{0} .
$$

It is well known that the above assumption yields that the system

$$
g_{1}(\mathbf{x})=\cdots=g_{m}(\mathbf{x})=0, \mathbf{x} \in \mathbb{C}^{m}
$$

has exactly $D=\prod_{i=1}^{m} d_{i}$ solutions, counted with multiplicities, and no solutions at infinity 23.

We now give a simple condition which imply that the system (41) has $D$ distinct solutions. 
Lemma 2. Let $g_{i}(\mathbf{x}), \mathbf{x}=\left(x_{1}, \ldots, x_{m}\right)^{\top} \in \mathbb{C}^{m}$ be a polynomial of degree $d_{i} \geqslant 1$ for $i \in[m]$. Assume that $D=\prod_{i=1}^{m} d_{i}>1$ and the system (41) satisfies the condition (40). Then the system (41) has $D$ distinct solutions if and only if the system

$$
g_{i}(\mathbf{x})=0, i \in[m], \quad \operatorname{det}\left[\frac{\partial g_{i}}{\partial x_{j}}(\mathbf{x})\right]_{i, j \in[m]}=0
$$

is not solvable over $\mathbb{C}^{n}$.

Proof. Let $\mathbf{G}=\left(g_{1}, \ldots, g_{m}\right): \mathbb{C}^{m} \rightarrow \mathbb{C}^{m}$. Recall that the assumptions that the system (41) satisfies the condition (40) implies that $G$ is a proper map. Assume that $\mathbf{G}(\zeta)=\mathbf{0}$. Then $\zeta$ is a simple solution of $G(\mathbf{x})=\mathbf{0}$ if and only if $\operatorname{det}\left[\frac{\partial g_{i}}{\partial x_{j}}(\mathbf{x})\right]_{i, j \in[m]} \neq 0$. Hence, if the system (41) has $D$ distinct solutions it follows that the above system is not solvable.

Vice versa, assume that the above system is not solvable. Then the Jacobian matrix $\mathrm{D}(\mathbf{G})$ is invertible at $\zeta$. Therefore $\mathbf{G}$ is a local invertible map in the neighborhood of $\zeta$. In particular $\zeta$ is a simple zero of $\mathbf{G}$. As $\mathbf{G}^{-1}(\mathbf{0})$ has $D$ points counting with their multipliciites, it follows that $\left|\mathbf{G}^{-1}(\mathbf{0})\right|=D$.

Definition 3. A system of polynomial equations (41) is called simple if the following conditions hold:

(1) For each $i \in[m]$ the inequality $d_{i}=\operatorname{deg} g_{i} \geqslant 1$ holds.

(2) The total degree of the system $D=\prod_{i=1}^{m} d_{i}$ is greater than 1 .

(3) The condition (40) holds.

(4) The system (41) has $D$ distinct solutions.

The system (41) is called $x_{1}$-simple if in addition to the above conditions the $x_{1}$-coordinates of $D$ solutions are distinct.

An example of a simple system system is

$$
\frac{1}{d_{i}} x_{i}^{d_{i}}-x_{i}=0, \quad 1<d_{i} \in \mathbb{N}, i \in[m]
$$

An example of $x_{1}$-simple system is obtained from the above system by replacing the $g_{1}(\mathbf{x})=\frac{1}{d_{1}} x_{1}^{d_{i}}-x_{1}$ with $g_{1}(\mathbf{x})=\frac{1}{d_{1}}\left(x_{1}+\sum_{i=2}^{m} a_{i} x_{i}\right)^{d_{1}}-x_{1}$. It is straightforward to show that this system is $x_{1}$-simple if and only if $\left(a_{2}, \ldots, a_{m}\right)^{\top}$ is not in a corresponding variety $V \subset \mathbb{C}^{m-1}$. Indeed, find all possible values of $x_{2}, \ldots, x_{m}$ to obtain the monic polynomial $p_{1}\left(x_{1}, a_{2}, \ldots, a_{m}\right)$ of degree $D$ that its zeros are the $x_{1}$-coordinates of the corresponding solution of this system. Then $p_{1}\left(x_{1}, a_{2}, \ldots, a_{m}\right)$ has $D$ simple solutions if and only if the discriminant of $p_{1}$ is not zero.

We now discuss briefly available methods to find all solutions of an $x_{1}$-simple system (41) and their arithmetic and bit complexities. Recall that arithmetic complexity counts the number of operations to execute a given algorithm, where each operation is counted as one unit of time. The bit complexity takes also in account the number of bits required for each operation, as addition/subtraction and product/division, where the length of the bits are count. Let $\mathcal{O}$ and $\mathcal{O}_{B}$ denote the arithmetic and the bit complexities respectively. By $\tilde{\mathcal{O}}$ and $\tilde{\mathcal{O}}_{B}$ we denote the arithmetic and the bit complexities, where some logarithm terms are dropped.

One of the standard method to solve system (41) is to use the reduced Gröbner basis with respect to an ordering of monomials that is preserved under product. See for example [5]. The simplest order is a lexicographical order $x_{1} \prec x_{2} \prec \cdots \prec x_{m}$. We now recall a simple case of the Shape Lemma that we are using in this paper [50]:

Lemma 4. (Shape Lemma) Let (41) be a simple system. Then the last polynomial in the reduced Gröbner basis is a monic polynomial $p_{1}\left(x_{1}\right)$ of degree $D$. If the system (41) is $x_{1}$-simple then the reduced Gröbner basis is of the form: $p_{1}\left(x_{1}\right), x_{2}-p_{2}\left(x_{1}\right), \ldots, x_{m}-p_{m}\left(x_{1}\right)$, and $\operatorname{deg} p_{i}<D$ for $i=2, \ldots, m$.

If in addition to the above assumptions $g_{1}, \ldots, g_{m}$ have real coefficients then $p_{1}, \ldots, p_{m}$ have real coefficients.

In the rest of this Appendix what we assume that the system (41) is $x_{1}$-simple. Then all solutions of (41) are of the form $\left(x_{1}, p_{2}\left(x_{1}\right), \ldots, p_{n}\left(x_{1}\right)\right)$, for all zeros $x_{1}$ of $p_{1}$. Assume furthermore that $g_{1}, \ldots, g_{n}$ have real coefficients. Then all real solutions of an $x_{1}$-simple systems are of the form $\left(x_{1}, p_{2}\left(x_{1}\right), \ldots, p_{m}\left(x_{1}\right)\right)$, for all real zeros $x_{1}$ of $p_{1}$. 
In what follows we consider an $x_{1}$-simple system of polynomial equations (41), where

$$
d_{1}=\cdots=d_{m}=d-1 \geqslant 2,
$$

with coefficients either in the domain of Gaussian integers $\mathbb{Z}[\mathbf{i}]$, or subdomain of integers $\mathbb{Z}$. Let $\tau-1$ be the maximal number of bit size of the coefficients of the monomials in $g_{1}, \ldots, g_{m}$. (Thus $\tau \in \mathbb{N}$.) For our purposes we assume that $m$ is fixed and the positive integer $d$ varies. First consider $p_{1}\left(x_{1}\right)$. What is the arithmetic complexity to compute $p_{1}$ ? Proposition 1 in [5] gives $\mathcal{O}\left(m d^{3 m-2}\right.$ ). (Assuming that the complexity of multiplication of two $p \times p$ matrices is $\mathcal{O}\left(p^{3}\right)$.) The bit complexity of finding $p_{1}\left(x_{1}\right)$ is $\tau$ times the arithmetic complexity times the size of the linear system to solve. The linear system is of order $m(d-1)^{m}$. Hence the bit complexity is $\mathcal{O}_{B}\left(\tau m^{2} d^{4 m-2}\right)$. We next discuss the maximum bit size of the coefficients of $p_{i}\left(x_{1}\right)$ for $i \in[m]$. The arguments of [42] yield that the maximum bit size is $\mathcal{O}\left(m(d-1)^{m}(\tau+\log d)\right)$.

We now recall the complexity results for computations of the roots of $p_{1}\left(x_{1}\right)$ with precision $2^{-\ell}$ for $\ell \in \mathbb{N}$. Consider a polynomial equation over $\mathbb{C}$ of degree $N \geqslant 2$ :

$$
f(x)=x^{N}+\sum_{i=1}^{N} a_{i} x^{N-i}=0, \quad a_{i} \in \mathbb{C}, i \in[N] .
$$

Assume that $\left|a_{i}\right|<2^{\beta}$ for $i \in[N]$ and $\beta \in \mathbb{N}$. Then the arithmetic complexity of computing all roots of the above equation with precision $2^{-\ell}$ is $\mathcal{O}\left(N \log ^{5} N \log (\max (N, \beta)+\ell)\right)$. The bit complexity is equal to the arithmetic complexity times $M\left(2 N^{2} \max (N, \beta)+\ell\right)$ [4]. Here

$$
M(t)=\mathcal{O}(t(\log t) \log \log t)
$$

is the complexity of multiplying two $t$ bit integers. That is, the bit complexity of computing all roots of $f$ with precision $2^{-\ell}$ is at most of order

$$
M\left(2 N^{2} \max (N, \beta)+\ell\right) N \log ^{5} N \log (\max (N, \beta)+\ell) .
$$

We now give the complexity of finding each coordinate of each solution of the system (41), satisfying the condition (43), with a relative precision $\varepsilon$ :

Lemma 5. Consider an $x_{1}$-simple system of polynomial equations with coefficients in $\mathbb{Z}[\mathbf{i}]$, which satisfies the condition (43). Let $\tau-1$ be the maximal number of bit size of the coefficients of the monomials in $g_{1}, \ldots, g_{m}$. Then the bit complexity of computing the value of each coordinate of each solution of the system with precision $2^{-\ell}, \ell \in \mathbb{N}$ is of at most of order

$$
\tau m^{2} d^{4 m-2}+M\left(2 N^{2} \max (N, \beta)+\ell^{\prime}\right) N \log ^{5} N \log \left(\beta+\ell^{\prime}\right)
$$

where

$$
N=(d-1)^{m}, \beta=\mathcal{O}\left(m(d-1)^{m}(\tau+\log d)\right), \ell^{\prime}=\ell+N(\beta+1) .
$$

Proof. We first compute $p_{1}\left(x_{1}\right)$. The bit complexity of computing $p_{1}\left(x_{1}\right) \mathcal{O}\left(\tau m^{2} d^{4 m-2}\right)$. Next we compute all roots of $p_{1}\left(x_{1}\right)$ with precision $2^{-\ell^{\prime}}$ for $\ell^{\prime} \geqslant \ell$ to be determined later. Recall that the degree of the monic polynomial $p_{1}\left(x_{1}\right)$ is $N=(d-1)^{m}$, and the maximum bits of its coefficients is bounded by $\beta-1$, where $\beta=\mathcal{O}\left(m(d-1)^{m}(\tau+\log d)\right)$. Hence the bit complexity of finding the roots of $p_{1}\left(x_{1}\right)$ is $\mathcal{O}\left(M\left(2 N^{2} \max (N, \beta)+\right.\right.$ $\left.\left.\ell^{\prime}\right) N \log ^{5} N \log \left(\beta+\ell^{\prime}\right)\right)$. Observe next that any root $z$ of $p_{1}\left(x_{1}\right)$ satisfies the inequality $|z|^{N} \leqslant 2^{\beta-1} \frac{|z|^{N}-1}{|z|-1}$. Hence $|z| \leqslant 2^{\beta}$. It is left to estimate the error in the $k(>1)$ coordinate of a solution $\zeta=\left(\zeta_{1}, \ldots, \zeta_{m}\right)$ of the system. Recall that $\zeta_{k}=p_{k}\left(\zeta_{1}\right), \operatorname{deg} p_{k}<N$, and the bit size of each coefficient of $p_{k}$ is at most $\beta-1$. Let $\eta_{1}$ an approximation of $\zeta_{1}$ that satisfies $\left|\zeta_{1}-\eta_{1}\right| \leqslant 2^{-\ell^{\prime}} \leqslant 1 / 2$. Then $\left|\eta_{1}\right| \leqslant\left|\zeta_{1}\right|+1 / 2 \leqslant 2^{\beta}+1 / 2$. It is straightforward to show that

$$
\left|p_{k}\left(\zeta_{1}\right)-p_{k}\left(\eta_{1}\right)\right| \leqslant 2^{\beta-1}\left|\zeta_{1}-\eta_{1}\right| \sum_{j=1}^{N-1} j \max \left(\left|\zeta_{1}\right|,\left|\eta_{1}\right|\right)^{j-1} \leqslant\left|\zeta_{1}-\eta_{1}\right| 2^{N(\beta+1)}<2^{N(\beta+1)-\ell^{\prime}} .
$$

Hence for $\ell^{\prime}=\ell+N(\beta+1)$ we obtain that $\eta_{k}=p_{k}\left(\eta_{1}\right)$ is an approximation of $\zeta_{k}$ with $2^{-\ell}$ precision. 
Appendix 3: The Majorana representation

The geometrical measure of entanglement for qubits is studied extensively in physics literature 3 , 33, 35. 39, 43, 54. In some papers, as 3, 43, the authors use the Majorana representation of symmetric qubits. The paper [3] provides examples of what the authors believe the most entangled symmetric qubits for $d=4, \ldots, 12$ based on Majorana representation. As most mathematicians are not familiar with Majorana representation, we descibe in a few sentences the mathematical concepts of Majorama representation for the interested reader. We also describe briefly the main ideas behind the examples in [3].

Recall that a qubit state is identified with the class of all vectors $(d=1)$ :

$$
\left\{\zeta \mathbf{x}: \mathbf{x}=\left(x_{1}, x_{2}\right)^{\top} \in \mathbb{C}^{2},\|\mathbf{x}\|=1, \zeta \in \mathbb{C},|\zeta|=1\right\} .
$$

Thus the set of all quibits $\Gamma$ can be identified with the complex projective space $\mathbb{P} \mathbb{C}^{2}$. $\Gamma$ can be also identified with the Riemann sphere $\mathbb{C} \cup\{\infty\}$. Indeed, associate with a qubit $\mathbf{x}=\left(x_{1}, x_{2}\right)^{\top}, x_{1} \neq 0$ a unique complex number $z=\frac{x_{2}}{x_{1}} \in \mathbb{C}$. The qubit $\mathbf{x}=\left(0, x_{2}\right),\left|x_{2}\right|=1$ corresponds to $z=\infty$. Recall that the set of $d$-symmetric quibit can be identified with the projective set $\mathbb{P}\left(\mathrm{S}^{d} \mathbb{C}^{2}\right)$. Equivalently, the set of $d$-symmetric quibit can be identified with $\mathbb{P}(\mathrm{P}(d, 2, \mathbb{C}))$. Take $f(\mathbf{x}) \in \mathrm{P}(d, 2, \mathbb{C}) \backslash\{0\}$. Then $f\left(x_{1}, x_{2}\right)$ is a product of $d$ linear forms $f\left(x_{1}, x_{2}\right)=\prod_{i=1}^{d}\left(\zeta_{1, i} x_{2}-\zeta_{i, 2} x_{1}\right)$. Thus, there exists a unique correspondence between $[f] \in \mathbb{P}(\mathrm{P}(d, 2, \mathbb{C}))$ and the $d$ points $\left[\left(\zeta_{1, i}, \zeta_{i, 2}\right)^{\top}\right] \in \mathbb{P C}^{2}, i \in[d]$. This is the Majorana representation [?, $\left.\S 4.4\right]$.

Next one replaces the Riemann sphere by the ordinary sphere $S^{2} \subset \mathbb{R}^{3}$. (For example perform a stereographic projection of the unit sphere onto the complex plane.) The geometric intuition suggests that the most entangled $d$-symmetric quibits correspond to the most evenly distributed $d$ points on $\mathrm{S}^{2}$. More precisely there are two optimal models:

Tóths problem: How $d$ points have to be distributed on the unit sphere so that the minimum distance of all pairs of points becomes maximal [56].

Thomsons problem: How $d$ point charges can be distributed on the surface of a sphere so that the potential energy is minimized [55].

However, in certain cases as shown in [3] the most entagled symmetric states are do not solve neither of the above problems.

Department of Mathematics, Statistics and Computer Science, University of Illinois, 851 South Morgan Street, Chicago, Illinois 60607-7045, USA

E-mail address: friedlan@uic.edu

Department of Mathematics, University of Texas at Arlington, 411 S. Nedderman Drive, 478 Pickard Hall, Arlington, Texas 76019-0408, USA

E-mail address: li.wang@uta.edu 